\title{
ARRANGEMENTS AND FROBENIUS LIKE STRUCTURES
}

\author{
ALEXANDER VARCHENKO* \\ Department of Mathematics, University of North Carolina at Chapel Hill \\ Chapel Hill, NC 27599-3250, USA
}

\begin{abstract}
We consider a family of generic weighted arrangements of $n$ hyperplanes in $\mathbb{C}^{k}$ and show that the Gauss-Manin connection for the associated hypergeometric integrals, the contravariant form on the space of singular vectors, and the algebra of functions on the critical set of the master function define a Frobenius like structure on the base of the family.

As a result of this construction we show that the matrix elements of the linear operators of the Gauss-Manin connection are given by the $2 k+1$-st derivatives of a single function on the base of the family, the function called the potential of second kind, see formula (6.46).

On considère une famille d'arrangements pondérés génériques de $n$ hyperplans dans $C^{k}$ et montre que la connexion de Gauss - Manin pour les intégrales hypergéométriques associées, la forme contravariante sur l'espace des vecteurs singuliers et l'algébre de fonctions sur l'ensemble des points critiques définissent une structure du type Frobenius sur la base de la famille.

Comme un résultat de cette construction nous montrons que les éléments matriciels des opérateurs linéaires de la connexion de Gauss - Manin sont donnés par les $(2 k+1)$-mes dérivées d'une seule fonction sur la base de la famille, cf. la formule (6.46).
\end{abstract}

\section{Contents}

1. Introduction

2. Arrangements

2.1. Affine arrangement

2.2. Orlik-Solomon algebra

2.3. Orlik-Solomon algebra as an algebra of differential forms

2.4. Weights

2.5. Space of flags, see SV]

2.6. Duality, see SV]

2.7. Contravariant map and form, see [SV]

2.8. Arrangement with normal crossings

2.9. If the weights of dense edges are nonzero

2.10. Master function

2.11. If the weights are unbalanced

2.12. Hessian and residue bilinear form

2.13. Canonical isomorphism and algebra structures on $\operatorname{Sing} \mathcal{F}^{k}(\mathcal{C})$

2.14. Change of variables and canonical isomorphism

3. A family of parallelly transported hyperplanes

3.1. An arrangement in $\mathbb{C}^{n} \times \mathbb{C}^{k}$

3.2. Discriminant

3.3. Good fibers and combinatorial connection

^E-mail: anv@email.unc.edu, supported in part by NSF grant DMS-1101508 and DMS-1362924 
3.4. Bad fibers

3.5. Operators $K_{j}(z): V \rightarrow V, j \in J$

3.6. Gauss-Manin connection on $\left(\mathbb{C}^{n}-\Delta\right) \times(\operatorname{Sing} V) \rightarrow \mathbb{C}^{n}-\Delta \quad 11$

3.7. Bundle of algebras $\quad 12$

3.8. Quantum integrable model of the arrangement $(\mathcal{C}(z), a) \quad 13$

3.9. A remark. Asymptotically flat sections $\quad 13$

3.10. Conformal blocks, period map, potential functions $\quad 14$

3.11. Tangent bundle and a Frobenius like structure $\quad 15$

4. Points on line $\quad 18$

4.1. An arrangement in $\mathbb{C}^{n} \times \mathbb{C} \quad 18$

4.2. Good fibers $\quad 19$

4.3. Operators $K_{j}(z): V \rightarrow V \quad 20$

4.4. Conformal blocks $\quad 20$

4.5. Canonical isomorphism and period map $\quad 21$

4.6. Contravariant map as the inverse to the canonical map $\quad 22$

4.7. Multiplication on $\operatorname{Sing} V$ and $(\operatorname{Sing} V)^{*} \quad 23$

4.8. Tangent morphism $\quad 23$

4.9. Multiplication and potential function of second kind $\quad 24$

4.10. Connections on the bundle $\sqcup_{z \in \mathbb{C}^{n}-\Delta} B^{*}(z) \rightarrow \mathbb{C}^{n}-\Delta$ defined in (4.33) 25

4.11. Functoriality $\quad 26$

4.12. Frobenius like structure $\quad 30$

5. Generic lines on plane $\quad 30$

5.1. An arrangement in $\mathbb{C}^{n} \times \mathbb{C}^{2} \quad 30$

5.2. Good fibers $\quad 31$

5.3. Operators $K_{i}(z): V \rightarrow V$

5.4. Conformal blocks

5.5. Algebra $A_{\Phi}(z) \quad 34$

5.6. Proof of Theorem 5.15 36

5.7. Contravariant map as the inverse to the canonical map 37

5.8. Corollaries of Theorem 5.15

5.9. Frobenius like structure 42

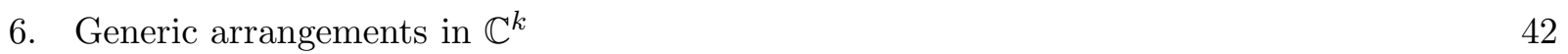

6.1. An arrangement in $\mathbb{C}^{n} \times \mathbb{C}^{k} \quad 42$

6.2. Good fibers $\quad 43$

6.3. Operators $K_{i}(z): V \rightarrow V \quad$ 44

6.4. Algebra $A_{\Phi}(z) \quad 45$

6.5. Canonical isomoprhism $\quad 50$

References 54

\section{InTRODUCTION}

There are three places, where a flat connection depending on a parameter appears: - KZ equations,

$$
\kappa \frac{\partial I}{\partial z_{i}}(z)=K_{i}(z) I(z), \quad z=\left(z_{1}, \ldots, z_{n}\right), \quad i=1, \ldots, n
$$


Here $\kappa$ is a parameter, $I(z)$ a $V$-valued function, where $V$ is a vector space from representation theory, $K_{i}(z): V \rightarrow V$ are linear operators, depending on $z$. The connection is flat for all $\kappa$. - Quantum differential equations,

$$
\kappa \frac{\partial I}{\partial z_{i}}(z)=p_{i} *_{z} I(z), \quad z=\left(z_{1}, \ldots, z_{n}\right), \quad i=1, \ldots, n
$$

Here $p_{1}, \ldots, p_{n}$ are generators of some commutative algebra $H$ with quantum multiplication $*_{z}$ depending on $z$. These equations are part of the Frobenius structure on the quantum cohomology of a variety.

- Differential equations for hypergeometric integrals associated with a family of weighted arrangements with parallelly transported hyperplanes,

$$
\kappa \frac{\partial I}{\partial z_{i}}(z)=K_{i}(z) I(z), \quad z=\left(z_{1}, \ldots, z_{n}\right), \quad i=1, \ldots, n
$$

It is well known that $\mathrm{KZ}$ equations are closely related with the differential equations for hypergeometric integrals. According to [SV] the KZ equations can be presented as equations for hypergeometric integrals for suitable arrangements. Thus (1.1) and (1.3) are related. Recently it was realized that in some cases the KZ equations appear as quantum differential equations, see [BMO] and [GRTV], and therefore the KZ equations are related to the Frobenius structures. On Frobenius structures see, for example, [D1, D2, M]. Hence (1.1) and (1.2) are related. In this paper I argue how a Frobenius like structure may appear on the base of a family of weighted arrangements. The goal is to make equations (1.3) related to Frobenius structures.

The main ingredients of a Frobenius structure are a flat connection depending on a parameter, a constant metric, a multiplication on tangent spaces. In our case, the connection comes from the differential equations for the associated hypergeometric integrals, the flat metric comes from the contravariant form on the space of singular vectors and the multiplication comes from the multiplication in the algebra of functions on the critical set of the master function. In this paper I consider the families of generic weighted arrangements.

The organization of the paper is as follows. In Section 2, objects associated with a weighted arrangement are recalled (Orlik-Solomon algebra, space of singular vectors, contravariant form, master function, canonical isomorphism of the space of singular vectors and the algebra of functions on the critical set of the master function). In Section 3, a family of arrangements with parallelly transported hyperplanes is considered. The construction of a Frobenius like structure on the base of the family is given. Conjectures [3.7, 3.8, 3.14 are formulated and corollaries of the conjectures are discussed. In Sections 4 and 5 the conjectures are proved for the family of points on the line and for a family of generic arrangements of lines on plane. The corresponding Frobenius like structures are described. Here are the corresponding potential functions of second kind:

$$
\tilde{P}\left(z_{1}, \ldots, z_{n}\right)=\frac{1}{2} \sum_{1 \leqslant i<j \leqslant n} a_{i} a_{j}\left(z_{i}-z_{j}\right)^{2} \log \left(z_{i}-z_{j}\right)
$$


for the family of arrangements of $n$ points on line and

$$
\tilde{P}\left(z_{1}, \ldots, z_{n}\right)=\frac{1}{4 !} \sum_{1 \leqslant i<j<k \leqslant n} \frac{a_{i} a_{j} a_{k}}{d_{i, j}^{2} d_{j, k}^{2} d_{k, i}^{2}}\left(z_{i} d_{j, k}+z_{j} d_{k, i}+z_{k} d_{i, j}\right)^{4} \log \left(z_{i} d_{j, k}+z_{j} d_{k, i}+z_{k} d_{i, j}\right)
$$

for the family of arrangements of $n$ generic lines on plane. The variables $z_{1}, \ldots, z_{n}$ are the parameters of the family, $a_{1}, \ldots, a_{n}$ are weights, $|a|=a_{1}+\cdots+a_{n}$, the number $d_{k, \ell}$ is the oriented area of the parallelogram generated by the normal vectors to the $k$-th and $\ell$-th lines, see formulas (4.36) and (6.44). Note that the potential $\tilde{P}$ from (1.4) appears in [D2] for $a_{1}=\cdots=a_{n}$ and in $\left[\mathrm{Ri}\right.$ for $a_{1}, \ldots, a_{n} \in \mathbb{Z}$.

In Section 6, the conjectures are proved for a family of generic arrangements of $n$ hyperplanes in $\mathbb{C}^{k}$ for any $k$. The potential $\tilde{P}\left(z_{1}, \ldots, z_{n}\right)$ of second kind is defined by formula (6.44) similar to formulas (1.4) and (1.5). It is shown that the matrix elements of the operators $K_{i}\left(z_{1}, \ldots, z_{n}\right)$ of the Gauss-Manin connection for associated hypergeometric integrals are given by the $2 k+1$-st derivatives of the potential of second kind, see formula (6.46).

This fact that the Gauss-Manin differential equations for associated hypergeometric integrals can be described in terms of derivatives of a single function on the base of the family is an important application of our Frobenius like structure. One may expect that this is a manifestation of a much more general phenomenon.

It should be stressed that that somewhat technical constructions in Section 3 are explained in details in Sections 4, 5, and 6 for the particular situations discussed there. The reader may decide to read first the easiest Section 4.

In this paper I followed one of I.M. Gelfand's rules: for a new subject, choose the simplest nontrivial example and write down everything explicitly for this example, see the introduction to [EFK].

I thank V. Schechtman and V. Tarasov for useful discussions.

\section{ArRangements}

2.1. Affine arrangement. Let $k, n$ be positive integers, $k<n$. Denote $J=\{1, \ldots, n\}$. Let $\mathcal{C}=\left(H_{j}\right)_{j \in J}$, be an arrangement of $n$ affine hyperplanes in $\mathbb{C}^{k}$. Denote $U=\mathbb{C}^{k}-\cup_{j \in J} H_{j}$, the complement. An edge $X_{\alpha} \subset \mathbb{C}^{k}$ of $\mathcal{C}$ is a nonempty intersection of some hyperplanes of $\mathcal{C}$. Denote by $J_{\alpha} \subset J$ the subset of indices of all hyperplanes containing $X_{\alpha}$. Denote $l_{\alpha}=\operatorname{codim}_{\mathbb{C}^{k}} X_{\alpha}$.

A subset $\left\{j_{1}, \ldots, j_{p}\right\} \subset J$ is called independent if the hyperplanes $H_{j_{1}}, \ldots, H_{j_{p}}$ intersect transversally.

We assume that $\mathcal{C}$ is essential, that is, $\mathcal{C}$ has a vertex. An edge is called dense if the subarrangement of all hyperplanes containing the edge is irreducible: the hyperplanes cannot be partitioned into nonempty sets so that, after a change of coordinates, hyperplanes in different sets are in different coordinates.

2.2. Orlik-Solomon algebra. Define complex vector spaces $\mathcal{A}^{p}(\mathcal{C}), p=0, \ldots, k$. For $p=0$ we set $\mathcal{A}^{p}(\mathcal{C})=\mathbb{C}$. For $p \geqslant 1, \mathcal{A}^{p}(\mathcal{C})$ is generated by symbols $\left(H_{j_{1}}, \ldots, H_{j_{p}}\right)$ with $j_{i} \in J$, such that 
(i) $\left(H_{j_{1}}, \ldots, H_{j_{p}}\right)=0$ if $H_{j_{1}}, \ldots, H_{j_{p}}$ are not in general position, that is, if the intersection $H_{j_{1}} \cap \ldots \cap H_{j_{p}}$ is empty or has codimension less than $p$;

(ii) $\left(H_{j_{\sigma(1)}}, \ldots, H_{j_{\sigma(p)}}\right)=(-1)^{|\sigma|}\left(H_{j_{1}}, \ldots, H_{j_{p}}\right)$ for any element $\sigma$ of the symmetric group $\Sigma_{p}$

(iii) $\sum_{i=1}^{p+1}(-1)^{i}\left(H_{j_{1}}, \ldots, \widehat{H}_{j_{i}}, \ldots, H_{j_{p+1}}\right)=0$ for any $(p+1)$-tuple $H_{j_{1}}, \ldots, H_{j_{p+1}}$ of hyperplanes in $\mathcal{C}$ which are not in general position and such that $H_{j_{1}} \cap \ldots \cap H_{j_{p+1}} \neq \emptyset$.

The direct sum $\mathcal{A}(\mathcal{C})=\oplus_{p=1}^{N} \mathcal{A}^{p}(\mathcal{C})$ is the (Orlik-Solomon) algebra with respect to multiplication

$$
\left(H_{j_{1}}, \ldots, H_{j_{p}}\right) \cdot\left(H_{j_{p+1}}, \ldots, H_{j_{p+q}}\right)=\left(H_{j_{1}}, \ldots, H_{j_{p}}, H_{j_{p+1}}, \ldots, H_{j_{p+q}}\right) .
$$

2.3. Orlik-Solomon algebra as an algebra of differential forms. For $j \in J$, fix a defining equation for the hyperplane $H_{j}, f_{j}=0$, where $f_{j}$ is a polynomial of degree one on $\mathbb{C}^{k}$. Consider the logarithmic differential form $\omega_{j}=d f_{j} / f_{j}$ on $\mathbb{C}^{k}$. Let $\overline{\mathcal{A}}(\mathcal{C})$ be the exterior $\mathbb{C}$-algebra of differential forms generated by 1 and $\omega_{j}, j \in J$. The map $\mathcal{A}(\mathcal{C}) \rightarrow$ $\overline{\mathcal{A}}(\mathcal{C}),\left(H_{j}\right) \mapsto \omega_{j}$, is an isomorphism. We identify $\mathcal{A}(\mathcal{C})$ and $\overline{\mathcal{A}}(\mathcal{C})$.

2.4. Weights. An arrangement $\mathcal{C}$ is weighted if a map $a: J \rightarrow \mathbb{C}^{\times}, j \mapsto a_{j}$, is given; $a_{j}$ is called the weight of $H_{j}$. For an edge $X_{\alpha}$, define its weight $a_{\alpha}=\sum_{j \in J_{\alpha}} a_{j}$.

Denote $\nu(a)=\sum_{j \in J} a_{j}\left(H_{j}\right) \in \mathcal{A}^{1}(\mathcal{C})$. Multiplication by $\nu(a)$ defines a differential $d^{(a)}$ : $\mathcal{A}^{p}(\mathcal{C}) \rightarrow \mathcal{A}^{p+1}(\mathcal{C}), x \mapsto \nu(a) \cdot x$, on $\mathcal{A}(\mathcal{C})$

2.5. Space of flags, see [SV]. For an edge $X_{\alpha}$ of codimension $l_{\alpha}=p$, a flag starting at $X_{\alpha}$ is a sequence

$$
X_{\alpha_{0}} \supset X_{\alpha_{1}} \supset \cdots \supset X_{\alpha_{p}}=X_{\alpha}
$$

of edges such that $l_{\alpha_{j}}=j$ for $j=0, \ldots, p$. For an edge $X_{\alpha}$, we define $\overline{\mathcal{F}}_{\alpha}$ as the complex vector space with basis vectors $\bar{F}_{\alpha_{0}, \ldots, \alpha_{p}=\alpha}$ labeled by the elements of the set of all flags starting at $X_{\alpha}$.

Define $\mathcal{F}_{\alpha}$ as the quotient of $\overline{\mathcal{F}}_{\alpha}$ by the subspace generated by all the vectors of the form

$$
\sum_{X_{\beta}, X_{\alpha_{j-1}} \supset X_{\beta} \supset X_{\alpha_{j+1}}} \bar{F}_{\alpha_{0}, \ldots, \alpha_{j-1}, \beta, \alpha_{j+1}, \ldots, \alpha_{p}=\alpha} .
$$

Such a vector is determined by $j \in\{1, \ldots, p-1\}$ and an incomplete flag $X_{\alpha_{0}} \supset \ldots \supset X_{\alpha_{j-1}} \supset$ $X_{\alpha_{j+1}} \supset \ldots \supset X_{\alpha_{p}}=X_{\alpha}$ with $l_{\alpha_{i}}=i$.

Denote by $F_{\alpha_{0}, \ldots, \alpha_{p}}$ the image in $\mathcal{F}_{\alpha}$ of the basis vector $\bar{F}_{\alpha_{0}, \ldots, \alpha_{p}}$. For $p=0, \ldots, k$, we set

$$
\mathcal{F}^{p}(\mathcal{C})=\oplus_{X_{\alpha}, l_{\alpha}=p} \mathcal{F}_{\alpha}
$$

2.6. Duality, see [SV]. The vector spaces $\mathcal{A}^{p}(\mathcal{C})$ and $\mathcal{F}^{p}(\mathcal{C})$ are dual. The pairing $\mathcal{A}^{p}(\mathcal{C}) \otimes$ $\mathcal{F}^{p}(\mathcal{C}) \rightarrow \mathbb{C}$ is defined as follows. For $H_{j_{1}}, \ldots, H_{j_{p}}$ in general position, we set $F\left(H_{j_{1}}, \ldots, H_{j_{p}}\right)=$ $F_{\alpha_{0}, \ldots, \alpha_{p}}$ where $X_{\alpha_{0}}=\mathbb{C}^{k}, \quad X_{\alpha_{1}}=H_{j_{1}}, \ldots, \quad X_{\alpha_{p}}=H_{j_{1}} \cap \cdots \cap H_{j_{p}}$. Then we define $\left\langle\left(H_{j_{1}}, \ldots, H_{j_{p}}\right), F_{\alpha_{0}, \ldots, \alpha_{p}}\right\rangle=(-1)^{|\sigma|}$, if $F_{\alpha_{0}, \ldots, \alpha_{p}}=F\left(H_{j_{\sigma(1)}}, \ldots, H_{j_{\sigma(p)}}\right)$ for some $\sigma \in \Sigma_{p}$, and $\left\langle\left(H_{j_{1}}, \ldots, H_{j_{p}}\right), F_{\alpha_{0}, \ldots, \alpha_{p}}\right\rangle=0$ otherwise. 
Define a map $\delta^{(a)}: \mathcal{F}^{p}(\mathcal{C}) \rightarrow \mathcal{F}^{p-1}(\mathcal{C})$ to be the map adjoint to $d^{(a)}: \mathcal{A}^{p-1}(\mathcal{C}) \rightarrow \mathcal{A}^{p}(\mathcal{C})$. An element $v \in \mathcal{F}^{k}(\mathcal{C})$ is called singular if $\delta^{(a)} v=0$. Denote by

$$
\operatorname{Sing} \mathcal{F}^{k}(\mathcal{C}) \subset \mathcal{F}^{k}(\mathcal{C})
$$

the subspace of all singular vectors.

2.7. Contravariant map and form, see [SV]. Weights $\left(a_{j}\right)_{j \in J}$ determine a contravariant map

$$
\mathcal{S}^{(a)}: \mathcal{F}^{p}(\mathcal{C}) \rightarrow \mathcal{A}^{p}(\mathcal{C}), \quad F_{\alpha_{0}, \ldots, \alpha_{p}} \mapsto \sum a_{j_{1}} \cdots a_{j_{p}}\left(H_{j_{1}}, \ldots, H_{j_{p}}\right)
$$

where the sum is taken over all $p$-tuples $\left(H_{j_{1}}, \ldots, H_{j_{p}}\right)$ such that

$$
H_{j_{1}} \supset X_{\alpha_{1}}, \ldots, H_{j_{p}} \supset X_{\alpha_{p}} .
$$

Identifying $\mathcal{A}^{p}(\mathcal{C})$ with $\mathcal{F}^{p}(\mathcal{C})^{*}$, we consider this map as a bilinear form,

$$
S^{(a)}: \mathcal{F}^{p}(\mathcal{C}) \otimes \mathcal{F}^{p}(\mathcal{C}) \rightarrow \mathbb{C}
$$

The bilinear form is called the contravariant form. The contravariant form is symmetric. For $F_{1}, F_{2} \in \mathcal{F}^{p}(\mathcal{C})$, we have

$$
S^{(a)}\left(F_{1}, F_{2}\right)=\sum_{\left\{j_{1}, \ldots, j_{p}\right\} \subset J} a_{j_{1}} \cdots a_{j_{p}}\left\langle\left(H_{j_{1}}, \ldots, H_{j_{p}}\right), F_{1}\right\rangle\left\langle\left(H_{j_{1}}, \ldots, H_{j_{p}}\right), F_{2}\right\rangle
$$

where the sum is over all unordered $p$-element subsets.

2.8. Arrangement with normal crossings. An essential arrangement $\mathcal{C}$ is with normal crossings, if exactly $k$ hyperplanes meet at every vertex of $\mathcal{C}$. Assume that $\mathcal{C}$ is an essential arrangement with normal crossings only. A subset $\left\{j_{1}, \ldots, j_{p}\right\} \subset J$ is called independent if the hyperplanes $H_{j_{1}}, \ldots, H_{j_{p}}$ intersect transversally.

A basis of $\mathcal{A}^{p}(\mathcal{C})$ is formed by $\left(H_{j_{1}}, \ldots, H_{j_{p}}\right)$ where $\left\{j_{1}<\cdots<j_{p}\right\}$ are independent ordered $p$-element subsets of $J$. The dual basis of $\mathcal{F}^{p}(\mathcal{C})$ is formed by the corresponding vectors $F\left(H_{j_{1}}, \ldots, H_{j_{p}}\right)$. These bases of $\mathcal{A}^{p}(\mathcal{C})$ and $\mathcal{F}^{p}(\mathcal{C})$ will be called standard.

We have

$$
F\left(H_{j_{1}}, \ldots, H_{j_{p}}\right)=(-1)^{|\sigma|} F\left(H_{j_{\sigma(1)}}, \ldots, H_{j_{\sigma(p)}}\right), \quad \sigma \in \Sigma_{p} .
$$

For an independent subset $\left\{j_{1}, \ldots, j_{p}\right\}$, we have

$$
S^{(a)}\left(F\left(H_{j_{1}}, \ldots, H_{j_{p}}\right), F\left(H_{j_{1}}, \ldots, H_{j_{p}}\right)\right)=a_{j_{1}} \cdots a_{j_{p}}
$$

and

$$
S^{(a)}\left(F\left(H_{j_{1}}, \ldots, H_{j_{p}}\right), F\left(H_{i_{1}}, \ldots, H_{i_{k}}\right)\right)=0
$$

for distinct elements of the standard basis. 


\subsection{If the weights of dense edges are nonzero.}

Theorem 2.1. Assume that the weights $\left(a_{j}\right)_{j \in J}$ are such that the weights of all dense edges of $\mathcal{C}$ are nonzero. Then

(i) the contravariant form is nondegenerate;

(ii) $H^{p}\left(\mathcal{A}^{*}(\mathcal{C}), d^{(a)}\right)=0$ for $p<k$ and $\operatorname{dim} H^{k}\left(\mathcal{A}^{*}, d^{(a)}\right)=|\chi(U)|$, where $\chi(U)$ is the Euler characteristics of $U$.

In particular, these statements hold if all the weights are positive.

Part (i) is proved in [SV]. Part (ii) is a straightforward corollary of results in [SV] as explained in Theorem 2.2 in [V6]. Part (ii) is proved in [Y], OT2].

2.10. Master function. Given weights $\left(a_{j}\right)_{j \in J}$, define the (multivalued) master function $\Phi: U \rightarrow \mathbb{C}$ by the formula:

$$
\Phi=\Phi_{\mathcal{C}, a}=\sum_{j \in J} a_{j} \log f_{j}
$$

A point $t \in U$ is a critical point if $\left.d \Phi\right|_{t}=\left.\nu(a)\right|_{t}=0$.

Theorem 2.2 ([V3, OT1, Si] $)$. For generic weights $\left(a_{j}\right)_{j \in J}$ all the critical points of $\Phi$ are nondegenerate and the number of critical points equals $|\chi(U)|$.

2.11. If the weights are unbalanced. Let $\mathcal{C}=\left(H_{j}\right)_{j \in J}$ be an essential arrangement in $\mathbb{C}^{k}$ with weights $\left(a_{j}\right)_{j \in J}$. Consider the compactification of the arrangement $\mathcal{C}$ in the projective space $\mathbb{P}^{k}$. Assign the weight $a_{\infty}=-\sum_{j \in J} a_{j}$ to the hyperplane $H_{\infty}=\mathbb{P}^{k}-\mathbb{C}^{k}$ and denote by $\hat{\mathcal{C}}$ the arrangement $\left(H_{j}\right)_{j \in J \cup \infty}$ in $\mathbb{P}^{k}$.

The weights of the arrangement $\mathcal{C}$ are called unbalanced if the weights of all the dense edges of $\hat{\mathcal{C}}$ are nonzero, see [V6]. For example, if all the weights $\left(a_{j}\right)_{j \in J}$ are positive, then the weights are unbalanced. The unbalanced weights form a Zarisky open subset in the space of all weight systems on $\mathcal{C}$.

Theorem 2.3 ([V6]). If the weights $a=\left(a_{j}\right)_{j \in J}$ of $\mathcal{C}$ are unbalanced, then all the critical points of the master function of the weighted arrangement $(\mathcal{C}, a)$ are isolated and the sum of Milnor numbers of all the critical points equals $|\chi(U)|$.

2.12. Hessian and residue bilinear form. Denote $\mathbb{C}(U)$ the algebra of rational functions on $\mathbb{C}^{k}$ regular on $U$ and $I_{\Phi}=\left\langle\frac{\partial \Phi}{\partial t_{i}} \mid i=1, \ldots, k\right\rangle \subset \mathbb{C}(U)$ the ideal generated by first derivatives of $\Phi$. Let

$$
A_{\Phi}=\mathbb{C}(U) / I_{\Phi}
$$

be the algebra of functions on the critical set and []: $\mathbb{C}(t)_{U} \rightarrow A_{\Phi}, f \mapsto[f]$, the canonical homomorphism.

If all critical points are isolated, then the critical set is finite and the algebra $A_{\Phi}$ is finitedimensional. In that case, $A_{\Phi}$ is the direct sum of local algebras corresponding to points $p$ of the critical set,

$$
A_{\Phi}=\oplus_{p} A_{p, \Phi}
$$

The local algebra $A_{p, \Phi}$ can be defined as the quotient of the algebra of germs at $p$ of holomorphic functions modulo the ideal $I_{p, \Phi}$ generated by first derivatives of $\Phi$. 
Lemma 2.4 ([V6]). The elements $\left[1 / f_{j}\right], j \in J$, generate $A_{\Phi}$.

We fix affine coordinates $t_{1}, \ldots, t_{k}$ on $\mathbb{C}^{k}$. Let

$$
f_{j}=b_{j}^{0}+b_{j}^{1} t_{1}+\cdots+b_{j}^{k} t_{k} .
$$

Lemma 2.5. The identity element $[1] \in A_{\Phi}(z)$ satisfies the equation

$$
[1]=\frac{1}{|a|} \sum_{j \in J} b_{j}^{0}\left[\frac{a_{j}}{f_{j}}\right]
$$

where $|a|=\sum_{j \in J} a_{j}$.

Proof. The lemma follows from the equality

$$
\sum_{i=1}^{k} t_{i} \frac{\partial \Phi}{\partial t_{i}}=|a|-\sum_{j \in J} b_{j}^{0}\left[\frac{a_{j}}{f_{j}}\right] .
$$

Surprisingly, formula (2.17) and Lemma 2.4 play central roles in the constructions of this paper.

We define the rational function Hess : $\mathbb{C}^{k} \rightarrow \mathbb{C}$, regular on $U$, by the formula

$$
\operatorname{Hess}(t)=\operatorname{det}_{1 \leqslant i, j \leqslant k}\left(\frac{\partial^{2} \Phi}{\partial t_{i} \partial t_{j}}\right)(t) .
$$

The function is called the Hessian of $\Phi$.

Let $\rho_{p}: A_{p, \Phi} \rightarrow \mathbb{C}$, be the Grothendieck residue,

$$
f \mapsto \frac{1}{(2 \pi \sqrt{-1})^{k}} \operatorname{Res}_{p} \frac{f}{\prod_{i=1}^{k} \frac{\partial \Phi}{\partial t_{i}}}=\frac{1}{(2 \pi \sqrt{-1})^{k}} \int_{\Gamma_{p}} \frac{f d t_{1} \wedge \cdots \wedge d t_{k}}{\prod_{i=1}^{k} \frac{\partial \Phi}{\partial t_{i}}},
$$

where $\Gamma_{p}$ is the real $k$ cycle in a small neighborhood of $p$, defined by the equations $\left|\frac{\partial \Phi}{\partial t_{i}}\right|=$ $\epsilon_{i}, i=1, \ldots, k$, and oriented by the condition $d \arg \frac{\partial \Phi}{\partial t_{1}} \wedge \cdots \wedge d \arg \frac{\partial \Phi}{\partial t_{k}}>0$, here $\epsilon_{s}$ are positive numbers sufficiently small with respect to the size of the neighborhood, see [GH, AGV].

Let $(,)_{p}$ be the residue bilinear form on $A_{p, \Phi}$,

$$
(f, g)_{p}=\rho_{p}(f g)
$$

for $f, g \in A_{p, \Phi}$. This form is nondegenerate.

Let all the critical points of $\Phi$ be isolated and hence, $A_{\Phi}=\oplus_{p} A_{p, \Phi}$. We define the residue bilinear form $($,$) on A_{\Phi}$ as $\oplus_{p}(,)_{p}$. This form is nondegenerate and $(f g, h)=(f, g h)$ for all $f, g, h \in A_{\Phi}$. In other words, the pair $\left(A_{\Phi},(),\right)$ is a Frobenius algebra.

2.13. Canonical isomorphism and algebra structures on $\operatorname{Sing} \mathcal{F}^{k}(\mathcal{C})$. Let $\left(F_{m}\right)_{m \in M}$ be a basis of $\mathcal{F}^{k}(\mathcal{C})$ and $\left(H^{m}\right)_{m \in M} \subset \mathcal{A}^{k}(\mathcal{C})$ the dual basis. Consider the element $\sum_{m} H^{m} \otimes F_{m} \in$ $\mathcal{A}^{k}(\mathcal{C}) \otimes \mathcal{F}^{k}(\mathcal{C})$. We have $H^{m}=f^{m} d t_{1} \wedge \cdots \wedge d t_{k}$ for some $f^{m} \in \mathbb{C}(U)$. The element

$$
E=\sum_{m \in M} f^{m} \otimes F_{m} \in \mathbb{C}(U) \otimes \mathcal{F}^{k}(\mathcal{C})
$$

is called the canonical element of $\mathcal{C}$. Denote $[E]$ the image of the canonical element in $A_{\Phi} \otimes \mathcal{F}^{k}(\mathcal{C})$. 
Theorem 2.6 ([V6]). We have $[E] \in A_{\Phi} \otimes \operatorname{Sing} \mathcal{F}^{k}(\mathcal{C})$.

Assume that all critical points of $\Phi$ are isolated. Introduce the linear map

$$
\alpha: A_{\Phi} \rightarrow \operatorname{Sing} \mathcal{F}^{k}(\mathcal{C}), \quad[g] \mapsto([g],[E]) .
$$

Theorem 2.7 ([V6] $)$. If the weights $\left(a_{j}\right)_{j \in J}$ of $\mathcal{C}$ are unbalanced, then the canonical map $\alpha$ is an isomorphism of vector spaces. The isomorphism $\alpha$ identifies the residue form on $A_{\Phi}$ and the contravariant form on $\operatorname{Sing} \mathcal{F}^{k}(\mathcal{C})$ multiplied by $(-1)^{k}$, that is,

$$
(f, g)=(-1)^{k} S^{(a)}(\alpha(f), \alpha(g)) \quad \text { for all } f, g \in A_{\Phi} .
$$

The map $\alpha$ is called the canonical map or canonical isomorphism.

Corollary 2.8 ([V6]). The restriction of the contravariant form $S^{(a)}$ to the subspace $\operatorname{Sing} \mathcal{F}^{k}(\mathcal{C})$ is nondegenerate.

On the restriction of the contravariant form $S^{(a)}$ to the subspace $\operatorname{Sing} \mathcal{F}^{k}(\mathcal{C})$ see $[\mathrm{FaV}]$.

If all critical points $p$ of the master function are nondegenerate, then

$$
\alpha:[g] \mapsto \sum_{p} \sum_{m} \frac{g(p) f^{m}(p)}{\operatorname{Hess}(p)} F_{m} .
$$

If the weights $\left(a_{j}\right)_{j \in J}$ of $\mathcal{C}$ are unbalanced, then the canonical isomorphism $\alpha: A_{\Phi} \rightarrow$ Sing $\mathcal{F}^{k}(\mathcal{C})$ induces a commutative associative algebra structure on $\operatorname{Sing} \mathcal{F}^{k}(\mathcal{C})$. Together with the contravariant form it is a Frobenius algebra structure.

2.14. Change of variables and canonical isomorphism. Assume that we change coordinates on $\mathbb{C}^{n}, t_{i}=\sum_{j=1}^{k} c_{i, j} s_{j}$ with $c_{i, j} \in \mathbb{C}$.

Lemma 2.9. The canonical map (2.22) in coordinates $t_{1}, \ldots, t_{k}$ equals the canonical map (2.22) in coordinates $s_{1}, \ldots, s_{k}$ divided by $\operatorname{det}\left(c_{i, j}\right), \alpha_{t}=\frac{1}{\operatorname{det}\left(c_{i, j}\right)} \alpha_{s}$.

Proof. We have $H^{m}=f^{m} d t_{1} \wedge \cdots \wedge d t_{k}=\operatorname{det}\left(c_{i, j}\right) f^{m} d s_{1} \wedge \cdots \wedge d s_{k}$ and $\operatorname{Hess}_{t}=\operatorname{det}^{2}\left(c_{i, j}\right) \operatorname{Hess}_{s}$. Now the lemma follows, for example, from (2.24).

To make the map (2.22) independent of coordinates one needs to consider it as a map

$$
A_{\Phi} \otimes d t_{1} \wedge \cdots \wedge d t_{k} \rightarrow \operatorname{Sing} \mathcal{F}^{k}(\mathcal{C}), \quad[g] \otimes d t_{1} \wedge \cdots \wedge d t_{k} \mapsto([g],[E]) .
$$

\section{A FAMILY OF PARALLELLY TRANSPORTED HYPERPLANES}

This section contains the main constructions of the paper. These constructions are explained in details in Sections 4, 5, and 6 for the particular situations discussed there.

3.1. An arrangement in $\mathbb{C}^{n} \times \mathbb{C}^{k}$. Recall that $J=\{1, \ldots, n\}$. Consider $\mathbb{C}^{k}$ with coordinates $t_{1}, \ldots, t_{k}, \mathbb{C}^{n}$ with coordinates $z_{1}, \ldots, z_{n}$, the projection $\mathbb{C}^{n} \times \mathbb{C}^{k} \rightarrow \mathbb{C}^{n}$. Fix $n$ nonzero linear functions on $\mathbb{C}^{k}, g_{j}=b_{j}^{1} t_{1}+\cdots+b_{j}^{k} t_{k}, j \in J$, where $b_{j}^{i} \in \mathbb{C}$. Define $n$ linear functions on $\mathbb{C}^{n} \times \mathbb{C}^{k}, f_{j}=z_{j}+g_{j}=z_{j}+b_{j}^{1} t_{1}+\cdots+b_{j}^{k} t_{k}, j \in J$. In $\mathbb{C}^{n} \times \mathbb{C}^{k}$ we define the arrangement $\tilde{\mathcal{C}}=\left\{\tilde{H}_{j} \mid f_{j}=0, j \in J\right\}$. Denote $\tilde{U}=\mathbb{C}^{n} \times \mathbb{C}^{k}-\cup_{j \in J} \tilde{H}_{j}$.

For every $z=\left(z_{1}, \ldots, z_{n}\right)$ the arrangement $\tilde{\mathcal{C}}$ induces an arrangement $\mathcal{C}(z)$ in the fiber of the projection over $z$. We identify every fiber with $\mathbb{C}^{k}$. Then $\mathcal{C}(z)$ consists of hyperplanes $H_{j}(z), j \in J$, defined in $\mathbb{C}^{k}$ by the equations $f_{j}=0$. Denote $U(\mathcal{C}(z))=\mathbb{C}^{k}-\cup_{j \in J} H_{j}(z)$, the 
complement to the arrangement $\mathcal{C}(z)$. We assume that for every $z$ the arrangement $\mathcal{C}(z)$ has a vertex. This happens if and only if $\mathcal{C}(0)$ has a vertex.

A point $z \in \mathbb{C}^{n}$ is called good if $\mathcal{C}(z)$ has normal crossings only. Good points form the complement in $\mathbb{C}^{n}$ to the union of suitable hyperplanes called the discriminant.

3.2. Discriminant. The collection $\left(g_{j}\right)_{j \in J}$ induces a matroid structure on $J$. A subset $C=\left\{i_{1}, \ldots, i_{r}\right\} \subset J$ is a circuit if $\left(g_{i}\right)_{i \in C}$ are linearly dependent but any proper subset of $C$ gives linearly independent $g_{i}$ 's.

For a circuit $C=\left\{i_{1}, \ldots, i_{r}\right\}$, let $\left(\lambda_{i}^{C}\right)_{i \in C}$ be a nonzero collection of complex numbers such that $\sum_{i \in C} \lambda_{i}^{C} g_{i}=0$. Such a collection is unique up to multiplication by a nonzero number.

For every circuit $C$ we fix such a collection and denote $f_{C}=\sum_{i \in C} \lambda_{i}^{C} z_{i}$. The equation $f_{C}=0$ defines a hyperplane $H_{C}$ in $\mathbb{C}^{n}$. It is convenient to assume that $\lambda_{i}^{C}=0$ for $i \in J-C$ and write $f_{C}=\sum_{i \in J} \lambda_{i}^{C} z_{i}$.

For any $z \in \mathbb{C}^{n}$, the hyperplanes $\left(H_{i}(z)\right)_{i \in C}$ in $\mathbb{C}^{k}$ have nonempty intersection if and only if $z \in H_{C}$. If $z \in H_{C}$, then the intersection has codimension $r-1$ in $\mathbb{C}^{k}$.

Denote by $\mathfrak{C}$ the set of all circuits in $J$. Denote $\Delta=\cup_{C \in \mathfrak{C}} H_{C}$. The arrangement $\mathcal{C}(z)$ in $\mathbb{C}^{k}$ has normal crossings if and only if $z \in \mathbb{C}^{n}-\Delta$, see [V6].

For example, if $k=1$ and $f_{j}=t_{1}+z_{j}, j \in J$, then the discriminant is the union of hyperplanes in $\mathbb{C}^{n}$ defined by the equations $z_{i}-z_{j}=0,1 \leqslant i<j \leqslant n$.

3.3. Good fibers and combinatorial connection. For any $z^{1}, z^{2} \in \mathbb{C}^{n}-\Delta$, the spaces $\mathcal{F}^{p}\left(\mathcal{C}\left(z^{1}\right)\right), \mathcal{F}^{p}\left(\mathcal{C}\left(z^{2}\right)\right)$ are canonically identified. Namely, a vector $F\left(H_{j_{1}}\left(z^{1}\right), \ldots, H_{j_{p}}\left(z^{1}\right)\right)$ of the first space is identified with the vector $F\left(H_{j_{1}}\left(z^{2}\right), \ldots, H_{j_{p}}\left(z^{2}\right)\right)$ of the second. In other words, we identify the standard bases of these spaces.

Assume that nonzero weights $\left(a_{j}\right)_{j \in J}$ are given. Then each arrangement $\mathcal{C}(z)$ is weighted. The identification of spaces $\mathcal{F}^{p}\left(\mathcal{C}\left(z^{1}\right)\right), \mathcal{F}^{p}\left(\mathcal{C}\left(z^{2}\right)\right)$ for $z^{1}, z^{2} \in \mathbb{C}^{n}-\Delta$ identifies the corresponding subspaces $\operatorname{Sing} \mathcal{F}^{k}\left(\mathcal{C}\left(z^{1}\right)\right)$, Sing $\mathcal{F}^{k}\left(\mathcal{C}\left(z^{2}\right)\right)$ and contravariant forms.

For a point $z \in \mathbb{C}^{n}-\Delta$, we denote $V=\mathcal{F}^{k}(\mathcal{C}(z))$, Sing $V=\operatorname{Sing} \mathcal{F}^{k}(\mathcal{C}(z))$. The triple $\left(V\right.$, Sing $\left.V, S^{(a)}\right)$ does not depend on $z \in \mathbb{C}^{n}-\Delta$ under the above identification.

As a result of this reasoning we obtain the canonically trivialized vector bundle

$$
\sqcup_{z \in \mathbb{C}^{n}-\Delta} \mathcal{F}^{k}(\mathcal{C}(z)) \rightarrow \mathbb{C}^{n}-\Delta,
$$

with the canonically trivialized subbundle $\sqcup_{z \in \mathbb{C}^{n-\Delta}} \operatorname{Sing} \mathcal{F}^{k}(\mathcal{C}(z)) \rightarrow \mathbb{C}^{n}-\Delta$ and the constant contravariant form on the fibers. This trivialization identifies the bundle in (3.1) with

$$
\left(\mathbb{C}^{n}-\Delta\right) \times V \rightarrow \mathbb{C}^{n}-\Delta
$$

and the subbundle with

$$
\left(\mathbb{C}^{n}-\Delta\right) \times(\operatorname{Sing} V) \rightarrow \mathbb{C}^{n}-\Delta .
$$

The bundle in (3.3) will be called the combinatorial bundle, the flat connection on it will be called combinatorial.

Lemma 3.1. If the weights $\left(a_{j}\right)_{j \in J}$ are unbalanced for the arrangement $\mathcal{C}(z)$ for some $z \in$ $\mathbb{C}^{n}-\Delta$, then the weights $\left(a_{j}\right)_{j \in J}$ are unbalanced for $\mathcal{C}(z)$ for all $z \in \mathbb{C}^{n}-\Delta$. 
3.4. Bad fibers. Points of $\Delta \subset \mathbb{C}^{n}$ are called bad. Let $z^{0} \in \Delta$ and $z \in \mathbb{C}^{n}-\Delta$. By definition, for any $p$ the space $\mathcal{A}^{p}\left(\mathcal{C}\left(z^{0}\right)\right)$ is obtained from $\mathcal{A}^{p}(\mathcal{C}(z))$ by adding new relations. Hence $\mathcal{A}^{k}\left(\mathcal{C}\left(z^{0}\right)\right)$ is canonically identified with the quotient space of $V^{*}=\mathcal{A}^{k}(\mathcal{C}(z))$ and $\mathcal{F}^{p}\left(\mathcal{C}\left(z^{0}\right)\right)$ is identified with a subspace of $V=\mathcal{F}^{p}(\mathcal{C}(z))$.

3.5. Operators $K_{j}(z): V \rightarrow V, j \in J$. For any circuit $C=\left\{i_{1}, \ldots, i_{r}\right\} \subset J$, we define the linear operator $L_{C}: V \rightarrow V$ as follows.

For $m=1, \ldots, r$, we define $C_{m}=C-\left\{i_{m}\right\}$. Let $\left\{j_{1}<\cdots<j_{k}\right\} \subset J$ be an independent ordered subset and $F\left(H_{j_{1}}, \ldots, H_{j_{k}}\right)$ the corresponding element of the standard basis. We define $L_{C}: F\left(H_{j_{1}}, \ldots, H_{j_{k}}\right) \mapsto 0$ if $\left|\left\{j_{1}, \ldots, j_{k}\right\} \cap C\right|<r-1$. If $\left\{j_{1}, \ldots, j_{k}\right\} \cap C=C_{m}$ for some $1 \leqslant m \leqslant r$, then by using the skew-symmetry property (2.9) we can write

$$
F\left(H_{j_{1}}, \ldots, H_{j_{k}}\right)= \pm F\left(H_{i_{1}}, H_{i_{2}}, \ldots, \widehat{H_{i_{m}}}, \ldots, H_{i_{r-1}} H_{i_{r}}, H_{s_{1}}, \ldots, H_{s_{k-r+1}}\right)
$$

with $\left\{s_{1}, \ldots, s_{k-r+1}\right\}=\left\{j_{1}, \ldots, j_{k}\right\}-C_{m}$. We set

$$
\begin{aligned}
& L_{C}: F\left(H_{i_{1}}, \ldots, \widehat{H_{i_{m}}}, \ldots, H_{i_{r}}, H_{s_{1}}, \ldots, H_{s_{k-r+1}}\right) \mapsto \\
&(-1)^{m} \sum_{l=1}^{r}(-1)^{l} a_{i_{l}} F\left(H_{i_{1}}, \ldots, \widehat{H_{i_{l}}}, \ldots, H_{i_{r}}, H_{s_{1}}, \ldots, H_{s_{k-r+1}}\right) .
\end{aligned}
$$

Consider on $\mathbb{C}^{n} \times \mathbb{C}^{k}$ the logarithmic differential one-forms $\omega_{C}=\frac{d f_{C}}{f_{C}}, C \in \mathfrak{C}$. Recall that $f_{C}=\sum_{j \in J} \lambda_{j}^{C} z_{j}$. We define

$$
K_{j}(z)=\sum_{C \in \mathfrak{C}} \frac{\lambda_{j}^{C}}{f_{C}(z)} L_{C}, \quad j \in J .
$$

The operators $K_{j}(z)$ are rational functions on $\mathbb{C}^{n}$ regular on $\mathbb{C}^{n}-\Delta$ and

$$
\sum_{C \in \mathfrak{C}} \omega_{C} \otimes L_{C}=\sum_{j \in J} d z_{j} \otimes K_{j}(z)
$$

Theorem 3.2 ([V6]). For any $j \in J$ and $z \in \mathbb{C}^{n}-\Delta$, the operator $K_{j}(z)$ preserves the subspace Sing $V \subset V$ and is a symmetric operator, $S^{(a)}\left(K_{j}(z) v, w\right)=S^{(a)}\left(v, K_{j}(z) w\right)$ for all $v, w \in V$.

3.6. Gauss-Manin connection on $\left(\mathbb{C}^{n}-\Delta\right) \times(\operatorname{Sing} V) \rightarrow \mathbb{C}^{n}-\Delta$. Consider the master function

$$
\Phi(z, t)=\sum_{j \in J} a_{j} \log f_{j}(z, t)
$$

as a function on $\tilde{U} \subset \mathbb{C}^{n} \times \mathbb{C}^{k}$. Let $\kappa \in \mathbb{C}^{\times}$. The function $e^{\Phi(z, t) / \kappa}$ defines a rank one local system $\mathcal{L}_{\kappa}$ on $\tilde{U}$ whose horizontal sections over open subsets of $\tilde{U}$ are univalued branches of $e^{\Phi(z, t) / \kappa}$ multiplied by complex numbers, see for example [SV, V2].

The vector bundle

$$
\sqcup_{z \in \mathbb{C}^{n}-\Delta} H_{k}\left(U(\mathcal{C}(z)),\left.\mathcal{L}_{\kappa}\right|_{U(\mathcal{C}(z))}\right) \rightarrow \mathbb{C}^{n}-\Delta
$$

will be called the homology bundle. The homology bundle has a canonical flat Gauss-Manin connection. 
For a fixed $z$, choose any $\gamma \in H_{k}\left(U(\mathcal{C}(z)),\left.\mathcal{L}_{\kappa}\right|_{U(\mathcal{C}(z))}\right)$. The linear map

$$
\{\gamma\}: \mathcal{A}^{k}(\mathcal{C}(z)) \rightarrow \mathbb{C}, \quad \omega \mapsto \int_{\gamma} e^{\Phi(z, t) / \kappa} \omega
$$

is an element of $\operatorname{Sing} \mathcal{F}^{k}(\mathcal{C}(z))$ by Stokes' theorem. It is known that for generic $\kappa$ any element of Sing $\mathcal{F}^{k}(\mathcal{C}(z))$ corresponds to a certain $\gamma$ and in that case this construction gives an isomorphism

$$
H_{k}\left(U(\mathcal{C}(z)),\left.\mathcal{L}_{\kappa}\right|_{U(\mathcal{C}(z))}\right) \rightarrow \operatorname{Sing} \mathcal{F}^{k}(\mathcal{C}(z)),
$$

see [SV]. This isomorphism will be called the integration isomorphism. The precise values of $\kappa$ for which (3.11) is an isomorphism can be deduced from the determinant formula in [V1].

For generic $\kappa$ the fiber isomorphisms (3.11) defines an isomorphism of the homology bundle and the combinatorial bundle. The Gauss-Manin connection induces a flat connection on the combinatorial bundle. This connection on the combinatorial bundle will be also called the Gauss-Manin connection.

Thus, there are two connections on the combinatorial bundle: the combinatorial connection and the Gauss-Manin connection depending on $\kappa$. In this situation we can consider the differential equations for flat sections of the Gauss-Manin connection with respect to the combinatorially flat standard basis. Namely, let $\gamma(z) \in H_{k}\left(U(\mathcal{C}(z)),\left.\mathcal{L}_{\kappa}\right|_{U(\mathcal{C}(z))}\right)$ be a flat section of the Gauss-Manin connection. Let us write the corresponding section $I_{\gamma}(z)$ of the bundle $\mathbb{C}^{n} \times \operatorname{Sing} V \rightarrow \mathbb{C}^{n}$ in the combinatorially flat standard basis,

$$
I_{\gamma}(z)=\sum_{\substack{\text { independent } \\\left\{j_{1}<\cdots<j_{k}\right\} \subset J}} I_{\gamma}^{j_{1}, \ldots, j_{k}}(z) F\left(H_{j_{1}}, \ldots, H_{j_{k}}\right), \quad I_{\gamma}^{j_{1}, \ldots, j_{k}}(z)=\int_{\gamma(z)} e^{\Phi(z, t) / \kappa} \omega_{j_{1}} \wedge \cdots \wedge \omega_{j_{k}} .
$$

For $I=\sum I^{j_{1}, \ldots, j_{k}} F\left(H_{j_{1}}, \ldots, H_{j_{k}}\right)$ and $j \in J$, we denote

$$
\frac{\partial I}{\partial z_{j}}=\sum \frac{\partial I^{j_{1}, \ldots, j_{k}}}{\partial z_{j}} F\left(H_{j_{1}}, \ldots, H_{j_{k}}\right) .
$$

Theorem 3.3 ([V2,, $\mathrm{V} 6])$. The section $I_{\gamma}(z)$ satisfies the differential equations

$$
\kappa \frac{\partial I}{\partial z_{j}}(z)=K_{j}(z) I(z), \quad j \in J
$$

where $K_{j}(z): V \rightarrow V$ are the linear operators defined in (3.6).

From this formula we see, in particular, that the combinatorial connection on the combinatorial bundle is the limit of the Gauss-Manin connection as $\kappa \rightarrow \infty$.

3.7. Bundle of algebras. For $z \in \mathbb{C}^{n}$, denote $A_{\Phi}(z)$ the algebra of functions on the critical set of the master function $\Phi(z, \cdot): U(\mathcal{C}(z)) \rightarrow \mathbb{C}$. Assume that the weights $\left(a_{j}\right)_{j \in J}$ are unbalanced for all $\mathcal{C}(z), z \in \mathbb{C}^{n}-\Delta$. Then the dimension of $A_{\Phi}(z)$ does not depend on $z \in \mathbb{C}^{n}-\Delta$ and equals $\operatorname{dim} \operatorname{Sing} V$. Denote $|a|=\sum_{j \in J} a_{j}$.

Lemma 3.4. The identity element $[1](z) \in A_{\Phi}(z)$ satisfies the equation

$$
[1](z)=\frac{1}{|a|} \sum_{j \in J} z_{j}\left[\frac{a_{j}}{f_{j}}\right] .
$$


Proof. The lemma follows from Lemma 2.5.

The vector bundle

$$
\sqcup_{z \in \mathbb{C}^{n}-\Delta} A_{\Phi}(z) \rightarrow \mathbb{C}^{n}-\Delta
$$

will be called the bundle of algebras of functions on the critical set. The fiber isomorphisms $(2.22)$,

$$
\alpha(z): A_{\Phi}(z) \rightarrow \operatorname{Sing} V
$$

establish an isomorphism $\alpha$ of the bundle of algebras and the combinatorial bundle. The isomorphism $\alpha$ and the connections on the combinatorial bundle (combinatorial and GaussManin connections) induce connections on the bundle of algebras which will be called also the combinatorial and Gauss-Manin connections on the bundle of algebras.

The canonical isomorphism $\alpha(z)$ induces a Frobenius algebra structure on $\operatorname{Sing} V$ which depends on $z$. The multiplication $*_{z}$ is described by the following theorem.

Theorem $3.5([\mathrm{~V} 6])$. The elements $\alpha(z)\left[\frac{a_{j}}{f_{j}}\right] \in \operatorname{Sing} V, j \in J$, generate the algebra. We have

$$
\alpha(z)\left[\frac{a_{j}}{f_{j}}\right] *_{z} v=K_{j}(z) v
$$

for all $v \in \operatorname{Sing} V$ and $j \in J$.

3.8. Quantum integrable model of the arrangement $(\mathcal{C}(z), a)$. For $z \in \mathbb{C}^{n}-\Delta$, the (commutative) subalgebra $\mathcal{B}(z) \subset \operatorname{End}(\operatorname{Sing} V)$ generated by $K_{j}(z), j \in J$, is called the algebra of geometric Hamiltonians, the triple ( $\left.\operatorname{Sing} V, S^{(a)}, \mathcal{B}(z)\right)$ is called the quantum integrable model of the weighted arrangement $(\mathcal{C}(z), a)$, see [V6].

The canonical isomorphism $\alpha(z)$ identifies the triple $\left(\operatorname{Sing} V, S^{(a)}, \mathcal{B}(z)\right)$ with the triple $\left(A_{\Phi}(z),(-1)^{k}(,)_{z}, A_{\Phi}(z)\right)$, see Theorems 2.7 and 3.5.

Notice that the operators $K_{j}(z)$ are defined in combinatorial terms, see Section 3.5, while the algebra $A_{\Phi}(z)$ is an analytic object, see (2.13). C.f. Corollaries 5.28 and 6.21 .

3.9. A remark. Asymptotically flat sections. Assume that the weights $\left(a_{j}\right)_{j \in J}$ are unbalanced for all $\mathcal{C}(z), z \in \mathbb{C}^{n}-\Delta$. Let $B \subset \mathbb{C}^{n}-\Delta$ be an open real $2 n$-dimensional ball. Let $\Psi: B \rightarrow \mathbb{C}$ be a holomorphic function. Let $s_{j}, j \in \mathbb{Z}_{\geqslant 0}$ be holomorphic sections over $B$ of the bundle of algebras, see (3.16). We say that

$$
s(z, \kappa)=e^{\Psi(z) / \kappa} \sum_{j \geqslant 0} \kappa^{j} s_{j}(z)
$$

is an asymptotically flat section of the Gauss-Manin connection on bundle of algebras as $\kappa \rightarrow 0$ if $s(z, \kappa)$ satisfies the flat section equations formally, see, for example, [RV, V5].

Assume that $B$ is such that for any $z \in B$, all the critical points of $\Phi(z, \cdot): U(\mathcal{C}(z)) \rightarrow \mathbb{C}$ are nondegenerate. Let us order them: $p_{1}(z), \ldots, p_{d}(z)$, where $d=\operatorname{dim} A_{\Phi}(z)=\operatorname{dim} \operatorname{Sing} V$. We may assume that every $p_{i}(z)$ depends on $z$ holomorphically. Then the function

$$
z \mapsto \operatorname{Hess}\left(z, p_{i}(z)\right)=\operatorname{det}_{1 \leqslant i, j \leqslant k}\left(\frac{\partial^{2} \Phi}{\partial t_{i} \partial t_{j}}\right)\left(z, p_{i}(z)\right)
$$


is a nonzero holomorphic function on $B$. We fix a square root $\operatorname{Hess}\left(z, p_{i}(z)\right)^{1 / 2}$. We denote $w_{i}(z)$ the element of $A_{\Phi}(z)$ which equals $\operatorname{Hess}\left(z, p_{i}(z)\right)^{1 / 2}$ at $p_{i}(z)$ and equals zero at all other critical points. Let $(,)_{z}$ be the residue form on $A_{\Phi}(z)$. Then

$$
\left(w_{i}(z), w_{j}(z)\right)_{z}=\delta_{i j} \quad \text { and } \quad w_{i}(z) \cdot w_{j}(z)=\delta_{i j} \operatorname{Hess}\left(z, p_{i}(z)\right)^{1 / 2} w_{i}(z)
$$

for all $i, j$.

Theorem 3.6. For every $i$, there exists a unique asymptotically flat section $s(z, \kappa)$ $=e^{\Psi(z) / \kappa} \sum_{j \geqslant 0} \kappa^{j} s_{j}(z)$ of the Gauss-Manin connection on the bundle of algebras such that

$$
\Psi(z)=\Phi\left(z, p_{i}(z)\right) \quad \text { and } \quad s_{0}(z)=w_{i}(z) .
$$

Proof. We first write asymptotically flat sections of the Gauss-Manin connection on the bundle $\left(\mathbb{C}^{n}-\Delta\right) \times \operatorname{Sing} V \rightarrow\left(\mathbb{C}^{n}-\Delta\right)$ by using the steepest descent method as in [RV, V5] and then observe that the leading terms of those sections are nothing else but $\alpha(z)\left(e^{\Phi\left(z, p_{i}(z)\right) / \kappa} w_{i}(z)\right)$.

3.10. Conformal blocks, period map, potential functions. Denote by

$$
\{1\}(z)=\alpha(z)([1](z))
$$

the identity element of the algebra structure on $\operatorname{Sing} V$ corresponding to a point $z \in \mathbb{C}^{n}-\Delta$. An analog of this element was studied in [MTV] in a situation related to the geometric Langlands correspondence, see element $v_{1}$ in [MTV, Section 8].

For $r<k$ and $m_{1}, \ldots, m_{r} \in J$, denote

$$
I_{m_{1}, \ldots, m_{r}}(z)=\frac{\partial^{r}\{1\}}{\partial z_{m_{1}} \ldots \partial z_{m_{r}}}(z) .
$$

Conjecture 3.7. The Sing $V$-valued function $\{1\}(z)$ satisfies the Gauss-Manin differential equations with parameter $\kappa=\frac{|a|}{k}$,

$$
\frac{|a|}{k} \frac{\partial\{1\}}{\partial z_{j}}(z)=K_{j}(z)\{1\}(z), \quad j \in J,
$$

where the derivatives are defined with respect to a combinatorially flat basis as in (3.13). More generally, for $r<k$ and $m_{1}, \ldots, m_{r} \in J$, the $\operatorname{Sing} V$-valued function $I_{m_{1}, \ldots, m_{r}}(z)$ satisfies the Gauss-Manin differential equations with parameter $\kappa=\frac{|a|}{k-r}$,

$$
\frac{|a|}{k-r} \frac{\partial I_{m_{1}, \ldots, m_{r}}}{\partial z_{j}}(z)=K_{j}(z) I_{m_{1}, \ldots, m_{r}}(z), \quad j \in J .
$$

Conjecture 3.8. If we write the Sing $V$-valued function $\{1\}(z)$ in coordinates with respect to a combinatorially flat basis, then $\{1\}(z)$ is a homogeneous polynomial in $z$ of degree $k$.

The conjectures describes the interrelations of four objects: the identity element in $A_{\Phi}(z)$, the canonical isomorphism, the integration isomorphism, and the Gauss-Manin connection on the homology bundle. In the next sections we will prove this conjecture for families of generic arrangements. 
Theorem 3.9. If Conjecture 3.7 holds, then for $r \leqslant k$ and $m_{1}, \ldots, m_{r} \in J$, we have

$$
\frac{\partial^{r}\{1\}}{\partial z_{m_{1}} \ldots \partial z_{m_{r}}}(z)=\frac{k(k-1) \ldots(k-r+1)}{|a|^{r}} \alpha(z)\left(\prod_{i=1}^{r}\left[\frac{a_{m_{i}}}{f_{m_{i}}}\right]\right) \text {. }
$$

Proof. The proof is by induction on $r$. For $r=0$, the statement is true: $\{1\}=\{1\}$. Assuming the statement is true for some $r$, we prove the statement for $r+1$. By (3.25) and Theorem 3.5, we have

$$
\begin{aligned}
& \frac{\partial^{r+1}\{1\}}{\partial z_{m_{1}} \ldots \partial z_{m_{r}} \partial z_{j}}(z)=\frac{k-r}{|a|} K_{j}(z) \frac{\partial^{r}\{1\}}{\partial z_{m_{1}} \ldots \partial z_{m_{r}}}(z)= \\
& =\frac{k-r}{|a|} \alpha(z)\left(\left[\frac{a_{j}}{f_{j}}\right]\right) *_{z} \frac{k(k-1) \ldots(k-r+1)}{|a|^{r}} \alpha(z)\left(\prod_{i=1}^{r}\left[\frac{a_{m_{i}}}{f_{m_{i}}}\right]\right)= \\
& =\frac{k(k-1) \ldots(k-r+1)(k-r)}{|a|^{r+1}} \alpha(z)\left(\left[\frac{a_{j}}{f_{j}}\right] \prod_{i=1}^{r}\left[\frac{a_{m_{i}}}{f_{m_{i}}}\right]\right) .
\end{aligned}
$$

For given $r<k$, the sections $I_{m_{1}, \ldots, m_{r}}(z), m_{1}, \ldots, m_{r} \in J$, generate a subbundle of the combinatorial bundle. We will call it the subbundle of conformal blocks at level $\frac{|a|}{k-r}$ and denote by $C B_{\frac{|a|}{k-r}}$. The subbundle of conformal blocks at level $\frac{|a|}{k-r}$ is invariant with respect to the Gauss-Manin connection with $\kappa=\frac{|a|}{k-r}$. On conformal blocks in conformal field theory see, for example, [FSV1, FSV2, V2] and Section 3.6 in [V4].

One may show that

$$
C B_{\frac{|a|}{k}} \subset C B_{\frac{|a|}{k-1}} \subset \cdots \subset C B_{\frac{|a|}{1}} .
$$

Let us consider $\operatorname{Sing} V$ as a complex manifold. At every point of $\operatorname{Sing} V$, the tangent space is identified with the vector space $\operatorname{Sing} V$. We will consider the manifold $\operatorname{Sing} V$ with the constant holomorphic metric defined by the contravariant form $S^{(a)}$. We will denote this metric by the same symbol $S^{(a)}$.

Define the period map $q: \mathbb{C}^{n}-\Delta \rightarrow \operatorname{Sing} V$ by the formula

$$
q: z \mapsto\{1\}(z)
$$

The period map is a polynomial map. Define the potential function of first kind $P$ : $\mathbb{C}^{n}-\Delta \rightarrow \mathbb{C}$, by the formula

$$
P(z)=S^{(a)}(q(z), q(z)) .
$$

The potential function of first kind is a polynomial.

3.11. Tangent bundle and a Frobenius like structure. Let $T\left(\mathbb{C}^{n}-\Delta\right) \rightarrow \mathbb{C}^{n}-\Delta$ be the tangent bundle on $\mathbb{C}^{n}-\Delta$. Denote $\partial_{j}=\frac{\partial}{\partial z_{j}}$ for $j \in J$. Consider the morphism $\beta$ of the tangent bundle to the bundle of algebras defined by the formula,

$$
\beta(z): \partial_{j} \in T_{z}\left(\mathbb{C}^{n}-\Delta\right) \mapsto\left[\frac{\partial \Phi}{\partial z_{j}}\right]=\left[\frac{a_{j}}{f_{j}}\right] \in A_{\Phi}(z) .
$$

The morphism $\beta$ will be called the tangent morphism. 
The residue form on the bundle of algebras induces a holomorphic bilinear form $\eta$ on fibers of the tangent bundle,

$$
\begin{aligned}
\eta\left(\partial_{i}, \partial_{j}\right)_{z} & =\left(\beta(z)\left(\partial_{i}\right), \beta(z)\left(\partial_{j}\right)\right)_{z}=(-1)^{k} S^{(a)}\left(\alpha(z) \beta(z)\left(\partial_{i}\right), \alpha(z) \beta(z)\left(\partial_{j}\right)\right)= \\
& =\left(\left[\frac{a_{i}}{f_{i}}\right],\left[\frac{a_{j}}{f_{j}}\right]\right)_{z}=(-1)^{k} S^{(a)}\left(\alpha(z)\left(\left[\frac{a_{i}}{f_{i}}\right]\right), \alpha(z)\left(\left[\frac{a_{j}}{f_{j}}\right]\right)\right) .
\end{aligned}
$$

Theorem 3.10. If Conjecture 3.7 holds, then the bilinear form $\eta$ is induced by the period map $q: \mathbb{C}^{n}-\Delta \rightarrow \operatorname{Sing} V$ from the flat metric $S^{(a)}$ multiplied by $(-1)^{k} \frac{|a|^{2}}{k^{2}}$,

$$
\eta\left(\partial_{i}, \partial_{j}\right)_{z}=\frac{|a|^{2}}{k^{2}}(-1)^{k} S^{(a)}\left(\frac{\partial q}{\partial z_{i}}(z), \frac{\partial q}{\partial z_{j}}(z)\right)
$$

Proof. By Theorem [3.9, we have $\frac{|a|}{k} \frac{\partial q}{\partial z_{j}}=\alpha(z)\left(\left[\frac{a_{j}}{f_{j}}\right]\right)$. Hence

$$
\frac{|a|^{2}}{k^{2}}(-1)^{k} S^{(a)}\left(\frac{\partial q}{\partial z_{i}}, \frac{\partial q}{\partial z_{j}}\right)=(-1)^{k} S^{(a)}\left(\alpha(z)\left(\left[\frac{a_{i}}{f_{i}}\right]\right), \alpha(z)\left(\left[\frac{a_{j}}{f_{j}}\right]\right)\right)=\eta\left(\partial_{i}, \partial_{j}\right)_{z}
$$

For $r \leqslant 2 k$, introduce the constant $A_{k, r}$ by the formula

$$
\begin{aligned}
& A_{k, r}=\sum_{i=0}^{r}\left(\begin{array}{l}
r \\
i
\end{array}\right) \frac{(k !)^{2}}{(k-i) !(k-r+i) !}, \quad \text { if } r \leqslant k, \\
& A_{k, r}=\sum_{i=r-k}^{k}\left(\begin{array}{l}
r \\
i
\end{array}\right) \frac{(k !)^{2}}{(k-i) !(k-r+i) !}, \quad \text { if } r>k .
\end{aligned}
$$

For example, $A_{2,3}=24$ and $A_{k, 2 k}=(2 k)$ !.

Theorem 3.11. If Conjectures 3.7 and 3.8 hold, then for any $r \leqslant 2 k$, we have

$$
\left(\beta(z)\left(\partial_{m_{1}}\right) *_{z} \cdots *_{z} \beta(z)\left(\partial_{m_{r}}\right),[1](z)\right)_{z}=\frac{(-1)^{k}|a|^{r}}{A_{k, r}} \frac{\partial^{r} P}{\partial z_{m_{1}} \ldots \partial z_{m_{r}}}(z),
$$

for all $m_{1}, \ldots, m_{r} \in J$. Here $(,)_{z}$ is the residue bilinear form on $A_{\Phi}(z)$.

Proof. We have

$$
\begin{gathered}
\left(\beta(z)\left(\partial_{m_{1}}\right) *_{z} \cdots *_{z} \beta(z)\left(\partial_{m_{r}}\right),[1](z)\right)_{z}=\left(\prod_{i=1}^{r}\left[\frac{a_{m_{i}}}{f_{m_{i}}}\right],[1](z)\right)_{z}= \\
=(-1)^{k} S^{(a)}\left(\alpha(z)\left(\prod_{i=1}^{r}\left[\frac{a_{m_{i}}}{f_{m_{i}}}\right]\right),\{1\}(z)\right) .
\end{gathered}
$$


Consider the example $r=2, k \geqslant 2$. Then

$$
\begin{aligned}
& \frac{\partial^{2}}{\partial z_{i} \partial z_{j}} S^{(a)}(q(z), q(z))=S^{(a)}\left(\frac{\partial^{2} q}{\partial z_{i} \partial z_{j}}, q\right)+S^{(a)}\left(\frac{\partial q}{\partial z_{i}}, \frac{\partial q}{\partial z_{j}}\right)+ \\
& +S^{(a)}\left(\frac{\partial q}{\partial z_{j}}, \frac{\partial q}{\partial z_{i}}\right)+S^{(a)}\left(q, \frac{\partial^{2} q}{\partial z_{i} \partial z_{j}}\right)=\frac{k(k-1)}{|a|^{2}} S^{(a)}\left(\alpha(z)\left(\left[\frac{a_{i}}{f_{i}}\right]\left[\frac{a_{i}}{f_{i}}\right]\right), q\right)+ \\
& +\frac{k^{2}}{|a|^{2}} S^{(a)}\left(\alpha(z)\left(\left[\frac{a_{i}}{f_{i}}\right]\right), \alpha(z)\left(\left[\frac{a_{j}}{f_{j}}\right]\right)\right)+\frac{k^{2}}{|a|^{2}} S^{(a)}\left(\alpha(z)\left(\left[\frac{a_{j}}{f_{j}}\right]\right), \alpha(z)\left(\left[\frac{a_{i}}{f_{i}}\right]\right)\right)+ \\
& +\frac{k(k-1)}{|a|^{2}} S^{(a)}\left(q, \alpha(z)\left(\left[\frac{a_{i}}{f_{i}}\right]\left[\frac{a_{i}}{f_{i}}\right]\right)\right)=\frac{A_{k, 2}}{|a|^{2}} S^{(a)}\left(\alpha(z)\left(\left[\frac{a_{i}}{f_{i}}\right]\left[\frac{a_{i}}{f_{i}}\right]\right), q\right) .
\end{aligned}
$$

Here we used Theorem 3.9 and the fact that $S^{(a)}$ is constant with respect to the combinatorial connection. This calculation proves the theorem for $r=2, k \geqslant 2$. The general case for $r \leqslant k$ is proved exactly in the same way. If $r>k$, then we need to take into account that $q(z)$ is a polynomial of degree $k$.

For $v \in \operatorname{Sing} V$, define the differential one-form $\psi_{v}$ on $\mathbb{C}^{n}-\Delta$ by the formula

$$
\psi_{v}: \partial_{i} \in T_{z}\left(\mathbb{C}^{n}-\Delta\right) \mapsto S^{(a)}\left(v, \alpha(z) \beta(z)\left(\partial_{i}\right)\right) .
$$

Theorem 3.12. If Conjecture 3.7 holds, then the differential form $\psi_{v}$ is exact,

$$
\psi_{v}=\frac{|a|}{k} d S^{(a)}(v, q(z))
$$

Proof. By Theorem [3.9, we have $\frac{|a|}{k} \frac{\partial q}{\partial z_{j}}=\alpha(z)\left(\left[\frac{a_{j}}{f_{j}}\right]\right)$. Hence

$\psi_{v}\left(\partial_{i}\right)=S^{(a)}\left(v, \alpha(z) \beta(z)\left(\partial_{i}\right)\right)=S^{(a)}\left(v, \alpha(z)\left(\left[\frac{a_{i}}{f_{i}}\right]\right)\right)=S^{(a)}\left(v, \frac{|a|}{k} \frac{\partial q}{\partial z_{j}}\right)=\frac{|a|}{k} \frac{\partial}{\partial z_{j}} S^{(a)}(v, q)$.

For $\kappa \in \mathbb{C}^{\times}$, let $I(z) \in \operatorname{Sing} V$ be a flat (multivalued) section of the Gauss-Manin connection with parameter $\kappa$. Define the (multivalued) differential one-form $\psi_{I}$ on $\mathbb{C}^{n}-\Delta$ by the formula

$$
\psi_{I}: \partial_{i} \in T_{z}\left(\mathbb{C}^{n}-\Delta\right) \mapsto S^{(a)}\left(I(z), \alpha(z) \beta(z)\left(\partial_{i}\right)\right) .
$$

Theorem 3.13. If Conjecture 3.7 holds and $\kappa \neq \frac{|a|}{k}$, then the differential form $\psi_{I}$ is exact,

$$
\psi_{I}=\left(\frac{1}{\kappa}+\frac{k}{|a|}\right)^{-1} d S^{(a)}(I(z), q(z)) .
$$

Proof. We have

$$
\begin{aligned}
& \frac{\partial}{\partial z_{j}} S^{(a)}(I(z), q(z))=S^{(a)}\left(\frac{\partial}{\partial z_{j}} I(z), q(z)\right)+S^{(a)}\left(I(z), \frac{\partial}{\partial z_{j}} q(z)\right)= \\
& \left.\quad=S^{(a)}\left(\frac{1}{\kappa} K_{j}(z) I(z), q(z)\right)+S^{(a)}\left(I(z), \frac{k}{|a|} K_{j}(z) q(z)\right)\right)= \\
& \quad=\left(\frac{1}{\kappa}+\frac{k}{|a|}\right) S^{(a)}\left(I(z), \alpha(z) \beta(z)\left(\left[\frac{a_{j}}{f_{j}}\right]\right)\right) .
\end{aligned}
$$


The functions $\mathbb{C}^{n}-\Delta \rightarrow \mathbb{C}, z \mapsto S^{(a)}(v, q(z))$, of Theorem 3.12 are nothing else but the coordinate functions of the period map. We will call them flat periods. The functions $\mathbb{C}^{n}-\Delta \rightarrow \mathbb{C}, z \mapsto S^{(a)}(I(z), q(z))$, of Theorem 3.13 will be called twisted periods.

Conjecture 3.14. There exists a function $\tilde{P}\left(z_{1}, \ldots, z_{n}\right)$ such that

$$
\frac{\partial^{2 k+1} \tilde{P}}{\partial z_{m_{0}} \cdots \partial z_{m_{2 k}}}(z)=(-1)^{k}\left(\beta(z)\left(\partial_{m_{0}}\right) *_{z} \cdots *_{z} \beta(z)\left(\partial_{m_{2 k}}\right),[1](z)\right)_{z}
$$

for all $m_{0}, \ldots, m_{2 k} \in J$.

The function $\tilde{P}(z)$ with this property will be called the potential function of second kind. Notice that formula (3.36) does not hold for $r=2 k+1$.

The potential function of second kind $\tilde{P}(z)$ determines the potential function of first kind $P(z)$. Indeed, by formula (3.4) we have

$$
P(z)=\frac{1}{|a|^{2 k+1}} \sum_{m_{0}, m_{1}, \ldots, m_{2 k} \in J} z_{m_{0}} z_{m_{1}} \ldots z_{m_{2 k}} \frac{\partial^{2 k+1} \tilde{P}}{\partial z_{m_{0}} \partial z_{m_{1}} \ldots \partial z_{m_{2 k}}}(z) .
$$

More generally, for any $r \leqslant 2 k$, we have

$$
\frac{\partial^{r} P}{\partial z_{m_{0}} \ldots \partial z_{m_{r-1}}}(z)=\frac{A_{k, r}}{|a|^{2 k+1}} \sum_{m_{r}, \ldots, m_{2 k} \in J} z_{m_{r}} \ldots z_{m_{2 k}} \frac{\partial^{2 k+1} \tilde{P}}{\partial z_{m_{0}} \ldots \partial z_{m_{2 k}}}(z) .
$$

We will call the collection of our objects - the combinatorial bundle $\left(\mathbb{C}^{n}-\Delta\right) \times(\operatorname{Sing} V) \rightarrow$ $\mathbb{C}^{n}-\Delta$ with the contravariant form $S^{(a)}$ and connections (combinatorial and Gauss-Manin); the bundle of algebras $\sqcup_{z \in \mathbb{C}^{n}-\Delta} A_{\Phi}(z) \rightarrow \mathbb{C}^{n}-\Delta$; the period map $q: \mathbb{C}^{n}-\Delta \rightarrow \operatorname{Sing} V$, the potential functions $P(z)$ and $\tilde{P}(z)$, flat periods $S^{(a)}(v, q(z))$, twisted periods $S^{(a)}(I(z), q(z))$ a Frobenius like structure on $\mathbb{C}^{n}-\Delta$.

The situation here reminds the structure induced on a submanifold of a Frobenius manifold, cf. [St]. From that point of view one may expect that $\operatorname{Sing} V$ has an honest Frobenius structure and our Frobenius like structure on $\mathbb{C}^{n}-\Delta$ is what can be induced from the Frobenius structure on $\operatorname{Sing} V$ by the period map, cf. with constructions in [Do, HM].

Numerous variations of the definition of the Frobenius structure see, for example, in [D1, D2, [M, St, FV].

In the next sections we will prove Conjectures 3.7, 3.8, 3.14 for families of generic arrangements and will describe our structure more precisely.

\section{Points ON LINE}

4.1. An arrangement in $\mathbb{C}^{n} \times \mathbb{C}$. Recall that $J=\{1, \ldots, n\}$. Consider $\mathbb{C}$ with coordinate $t$ and $\mathbb{C}^{n}$ with coordinates $z_{1}, \ldots, z_{n}$. Consider $n$ linear functions on $\mathbb{C}^{n} \times \mathbb{C}, f_{j}=z_{j}+t$, $j \in J$. In $\mathbb{C}^{n} \times \mathbb{C}$ we define the arrangement $\tilde{\mathcal{C}}=\left\{\tilde{H}_{j} \mid f_{j}=0, j \in J\right\}$.

For every $z=\left(z_{1}, \ldots, z_{n}\right) \in \mathbb{C}^{n}$ the arrangement $\tilde{\mathcal{C}}$ induces an arrangement $\mathcal{C}(z)$ in the fiber over $z$ of the projection $\mathbb{C}^{n} \times \mathbb{C} \rightarrow \mathbb{C}^{n}$. We identify the fiber with $\mathbb{C}$. The arrangement $\mathcal{C}(z)$ is the arrangement of points $\left\{-z_{1}, \ldots,-z_{n}\right\}$. Denote $U(\mathcal{C}(z))=\mathbb{C}-\left\{-z_{1}, \ldots,-z_{n}\right\}$ the complement. 
A point $z \in \mathbb{C}^{n}$ is good if the points $-z_{1}, \ldots,-z_{n}$ are distinct. Good points form the complement in $\mathbb{C}^{n}$ to the discriminant $\Delta$, which is the union of hyperplanes $H_{i j}=\left\{\left(z_{1}, \ldots, z_{n}\right) \in\right.$ $\left.\mathbb{C}^{n} \mid z_{i}=z_{j}\right\}$ labeled by two-element subsets $\{i, j\} \subset J$.

4.2. Good fibers. For any $z \in \mathbb{C}^{n}-\Delta$, the space $\mathcal{A}^{1}(\mathcal{C}(z))$ has the standard basis $H_{1}(z)$, $\ldots, H_{n}(z)$, the space $\mathcal{F}^{1}(\mathcal{C}(z))$ has the standard dual basis $F\left(H_{1}(z)\right), \ldots, F\left(H_{n}(z)\right)$. For $z^{1}, z^{2} \in \mathbb{C}^{n}-\Delta$, the combinatorial connection identifies the spaces $\mathcal{A}^{1}\left(\mathcal{C}\left(z^{1}\right)\right), \mathcal{F}^{1}\left(\mathcal{C}\left(z^{1}\right)\right)$ with the spaces $\mathcal{A}^{1}\left(\mathcal{C}\left(z^{2}\right)\right), \mathcal{F}^{1}\left(\mathcal{C}\left(z^{2}\right)\right)$, respectively, by identifying the corresponding standard bases.

Assume that nonzero weights $\left(a_{j}\right)_{j \in J}$ are given. Then each arrangement $\mathcal{C}(z)$ is weighted. For $z \in \mathbb{C}^{n}-\Delta$, the arrangement $\mathcal{C}(z)$ is unbalanced if $|a|=\sum_{j \in J} a_{j} \neq 0$. We assume $|a| \neq 0$.

For $z \in \mathbb{C}^{n}-\Delta$, we denote $V=\mathcal{F}^{1}(\mathcal{C}(z))$. We also denote $F_{j}=F\left(H_{j}(z)\right)$ for $j \in J$. We have

$$
S^{(a)}\left(F_{i}, F_{j}\right)=\delta_{i j} a_{i}, \quad \operatorname{Sing} V=\left\{\sum_{j \in J} c_{j} F_{j} \mid \sum_{j \in J} c_{j} a_{j}=0\right\} .
$$

For $j \in J$, we define the vector $v_{j} \in V$ by the formula

$$
v_{j}=-F_{j}+\frac{a_{j}}{|a|} \sum_{i \in J} F_{i} .
$$

Lemma 4.1. We have the following properties.

(i) $\operatorname{dim} \operatorname{Sing} V=n-1$.

(ii) For $j \in J$, we have $v_{j} \in \operatorname{Sing} V$ and $\sum_{j \in J} v_{j}=0$.

(iii) Any $n-1$ vectors of $\left(v_{j}\right)_{j \in J}$ are linearly independent.

(iv) We have

$$
\begin{aligned}
& S^{(a)}\left(v_{j}, v_{j}\right)=a_{j}-\frac{a_{j}^{2}}{|a|}, \quad j \in J, \\
& S^{(a)}\left(v_{i}, v_{j}\right)=-\frac{a_{i} a_{j}}{|a|}, \quad i, j \in J, i \neq j .
\end{aligned}
$$

Lemma 4.2. We have

$$
\operatorname{det}_{1 \leqslant i, j \leqslant n-1}\left(S^{(a)}\left(v_{i}, v_{j}\right)\right)=\frac{1}{|a|} \prod_{j \in J} a_{j} .
$$

Proof. Denote $M$ the transition matrix from the standard basis $F_{1}, \ldots, F_{n}$ of $V$ to the basis $v_{1}, \ldots, v_{n-1}, \sum_{j \in J} F_{j}$. It is easy to see that $\operatorname{det} M=(-1)^{n-1}$. The vector $\sum_{j \in J} F_{j}$ is orthogonal to $\operatorname{Sing} V$ and $S^{(a)}\left(\sum_{j \in J} F_{j}, \sum_{j \in J} F_{j}\right)=|a|$. The determinant of $S^{(a)}$ on $V$ with respect to the standard basis $F_{1}, \ldots, F_{n}$ equals $\prod_{j \in J} a_{j}$. These remarks imply (4.4). 
4.3. Operators $K_{j}(z): V \rightarrow V$. For any pair $\{i, j\} \subset J$, we define the linear operator $L_{i, j}: V \rightarrow V$ by the formula

$$
F_{i} \mapsto a_{j} F_{i}-a_{i} F_{j}, \quad F_{j} \mapsto a_{i} F_{j}-a_{j} F_{i}, \quad F_{m} \mapsto 0, \quad \text { if } m \notin\{i, j\},
$$

see formula (3.5). Define the operators $K_{j}(z): V \rightarrow V, j \in J$, by the formula

$$
K_{j}(z)=\sum_{i \neq j} \frac{L_{j, i}}{z_{j}-z_{i}}
$$

see formula (3.6). For any $j \in J$ and $z \in \mathbb{C}^{n}-\Delta$, the operator $K_{j}(z)$ preserves the subspace Sing $V \subset V$ and is a symmetric operator, that is $S^{(a)}\left(K_{j}(z) v, w\right)=S^{(a)}\left(v, K_{j}(z) w\right)$ for all $v, w \in V$, see Theorem 3.2 .

Lemma 4.3. For $j \in J$, we have

$$
\begin{aligned}
K_{j}(z) v_{i} & =\frac{a_{j}}{z_{j}-z_{i}} v_{i}+\frac{a_{i}}{z_{i}-z_{j}} v_{j}, \quad i \neq j \\
K_{j}(z) v_{j} & =-\sum_{i \neq j} K_{j}(z) v_{i} .
\end{aligned}
$$

Corollary 4.4. We have $K_{j}(z) v_{i}=K_{i}(z) v_{j}$ for all $i, j$.

The differential equations (3.14) for flat sections of the Gauss-Manin connection on $\left(\mathbb{C}^{n}-\Delta\right) \times \operatorname{Sing} V \rightarrow \mathbb{C}^{n}-\Delta$ take the form

$$
\kappa \frac{\partial I}{\partial z_{j}}(z)=K_{j}(z) I(z), \quad j \in J .
$$

For generic $\kappa$ all the flat sections are given by the formula

$$
I_{\gamma}(z)=\sum_{i \in J}\left(\int_{\gamma(z)} \prod_{m \in J}\left(z_{m}+t\right)^{a_{m} / \kappa} \frac{d t}{z_{i}+t}\right) F_{i},
$$

see formula (3.12). More precisely, all the flat sections are given by (4.9) if $1+\frac{|a|}{\kappa} \notin \mathbb{Z}_{\leqslant 0}$ and $1+\frac{a_{j}}{\kappa} \notin \mathbb{Z}_{\leqslant 0}$ for all $j \in J$, see [V1] or Theorem 3.3.5 in [V4].

Notice that equations (4.8) are a particular case of the KZ equations, see Section 1.1-1.3 in [V4].

\subsection{Conformal blocks.}

Lemma 4.5. If $\kappa=|a|$, then the Gauss-Manin connection has a one-dimensional invariant subbundle, generated by the section

$$
q: z \mapsto \frac{1}{|a|} \sum_{j \in J} z_{j} v_{j}=\frac{1}{|a|} \sum_{j \in J} q_{j}(z) F_{j},
$$

where

$$
q_{i}(z)=-z_{i}+\sum_{j \in J} \frac{a_{j}}{|a|} z_{j}
$$

This section is flat. 
Proof. The lemma follows from formulas (4.7).

This one-dimensional subbundle will be called the bundle of conformal blocks at level $|a|$. A flat section of the subbundle of conformal blocks can be presented as an integral $I_{\gamma}(z)$, where $\gamma$ is a small circle around infinity.

4.5. Canonical isomorphism and period map. The master function of the arrangement $\mathcal{C}(z)$ is

$$
\Phi(z, t)=\sum_{j \in J} a_{j} \log f_{j}=\sum_{j \in J} a_{j} \log \left(z_{j}+t\right) .
$$

The critical point equation is $\frac{\partial \Phi}{\partial t}=\sum_{j \in J} \frac{a_{j}}{z_{j}+t}=0$. The critical set is

$$
C_{\Phi}(z)=\left\{t \in U(\mathcal{C}(z)) \mid \sum_{j \in J} \frac{a_{j}}{z_{j}+t}=0\right\} .
$$

The algebra functions on the critical set is

$$
A_{\Phi}(z)=\mathbb{C}(U(\mathcal{C}(z))) /\left\langle\sum_{j \in J} \frac{a_{j}}{z_{j}+t}\right\rangle .
$$

The identity element $[1](z) \in A_{\Phi}(z)$ equals $\frac{1}{|a|} \sum_{j \in J} z_{j}\left[\frac{a_{j}}{f_{j}}\right]$.

Lemma 4.6. We have $\operatorname{dim} A_{\Phi}(z)=n-1$. Any $n-1$ elements of $\left(\left[\frac{a_{j}}{z_{j}+t}\right]\right)_{j \in J}$ are linearly independent.

Let $p \in C_{\Phi}(z)$. The Grothendieck residue $\rho_{p}: A_{p, \Phi}(z) \rightarrow \mathbb{C}$ is given by

$$
f \mapsto \frac{1}{2 \pi \sqrt{-1}} \operatorname{Res}_{p} \frac{f}{\frac{\partial \Phi}{\partial t}}=\frac{1}{2 \pi \sqrt{-1}} \int_{\Gamma_{p}} \frac{f d t}{\frac{\partial \Phi}{\partial t}},
$$

where $\Gamma_{p}$ is a small circle around the critical point $p$ oriented clock-wise. The residue bilinear form $(,)_{z}$ on $A_{\Phi}(z)$ is $\oplus_{p \in C_{\Phi}(z)}(,)_{p}$.

Lemma 4.7. For $f, g \in \mathbb{C}(U(\mathcal{C}(z)))$, we have

$$
([f],[g])=-\frac{1}{2 \pi \sqrt{-1}} \operatorname{Res}_{t=\infty} \frac{f g}{\frac{\partial \Phi}{\partial t}}-\frac{1}{2 \pi \sqrt{-1}} \sum_{i \in J} \operatorname{Res}_{t=-z_{i}} \frac{f g}{\frac{\partial \Phi}{\partial t}} .
$$

The canonical element is

$$
[E]=\sum_{j \in J}\left[\frac{1}{z_{j}+t}\right] \otimes F_{j} \quad \in \quad A_{\Phi}(z) \otimes \operatorname{Sing} V .
$$

The canonical isomorphism $\alpha(z): A_{\Phi}(z) \rightarrow \operatorname{Sing} V$ is given by the formula

$$
[f] \mapsto-\frac{1}{2 \pi \sqrt{-1}} \sum_{j \in J}\left(\operatorname{Res}_{t=\infty} \frac{f}{\left(z_{j}+t\right) \frac{\partial \Phi}{\partial t}}+\sum_{i \in J} \operatorname{Res}_{t=-z_{i}} \frac{f}{\left(z_{j}+t\right) \frac{\partial \Phi}{\partial t}}\right) F_{j} .
$$

Theorem 4.8. For $k \in J$, we have

$$
\alpha(z):\left[\frac{a_{k}}{z_{k}+t}\right] \mapsto v_{k}
$$


Proof. Denote

$$
g_{k j}=\frac{a_{k}}{\left(z_{k}+t\right)\left(z_{j}+t\right)} \frac{1}{\frac{\partial \Phi}{\partial t}}=\frac{a_{k}}{\left(z_{k}+t\right)\left(z_{j}+t\right)} \frac{\prod_{m \in J}\left(z_{m}+t\right)}{\sum_{m \in J} a_{m} \prod_{\ell \neq m}\left(z_{\ell}+t\right)} .
$$

If $k \neq j$, then $\operatorname{Res}_{t=-z_{i}} g_{j k}=0$ for all $i \in J$. If $k=j$, then $\operatorname{Res}_{t=-z_{i}} g_{j j}=0$ for $i \neq j$ and $\operatorname{Res}_{t=-z_{j}} g_{j j}=2 \pi \sqrt{-1}$. We also have $\operatorname{Res}_{t=\infty} g_{k j}=-2 \pi \sqrt{-1} \frac{a_{k}}{|a|}$ for all $j \in J$. We obtain the theorem by comparing these formulas with formula (4.2).

Corollary 4.9. Conjectures 3.7 and 3.8 hold for this family of arrangements.

Proof. By Theorem 4.8, we have $\alpha(z)([1](z))=q(z)$, where $q(z)$ is given by (4.10). Lemma 4.5 implies Conjectures 3.7 and 3.8 .

Corollary 4.10. For this family of arrangements the period map $q: \mathbb{C}^{n}-\Delta \rightarrow \operatorname{Sing} V$ is given by the formula

$$
q(z)=\frac{1}{|a|} \sum_{j \in J} z_{j} v_{j}=\frac{1}{|a|} \sum_{j \in J} q_{j}(z) F_{j},
$$

the potential function of first kind is

$$
P(z)=\frac{1}{|a|^{2}} \sum_{j \in J} a_{j} q_{j}^{2}(z)=\sum_{1 \leqslant i<j \leqslant n} \frac{a_{1} a_{2}}{|a|^{3}}\left(z_{i}-z_{j}\right)^{2} .
$$

By Corollary 4.10, the period map extends to a linear map $\mathbb{C}^{n} \rightarrow \operatorname{Sing} V$. The linear map is an epimorphism. The kernel is generated by the vector $(1, \ldots, 1)$.

The standard basis $\left(H_{j}\right)_{j \in J} \in V^{*}$ induces linear functions on $\operatorname{Sing} V$,

$$
h_{j}: v_{i} \mapsto \frac{a_{i}}{|a|}, \quad \text { if } j \neq i, \quad v_{j} \mapsto-1+\frac{a_{j}}{|a|} .
$$

We have $\sum_{j \in J} a_{j} h_{j}=0$ and any $n-1$ of these functions form a basis of $(\operatorname{Sing} V)^{*}$.

For $i \neq j$, define the hyperplane $\tilde{H}_{i, j} \subset \operatorname{Sing} V$ by the equation $h_{i}-h_{j}=0$.

Lemma 4.11. For all $i, j$ we have $q^{*}\left(h_{i}-h_{j}\right)=z_{j}-z_{i}$ and $q(\Delta)=\cup_{i<j} \tilde{H}_{i, j}$.

4.6. Contravariant map as the inverse to the canonical map. The canonical map $\alpha(z): A_{\Phi}(z) \rightarrow \operatorname{Sing} V$ is the isomorphism described in Theorem 4.8. The contravariant map $\mathcal{S}^{(a)}: V \rightarrow V^{*}$ is defined by the formula $F_{i} \mapsto a_{i}\left(H_{i}\right)$. By identifying $a_{i}\left(H_{i}\right)$ with the differential form $\frac{a_{i}}{f_{i}} d t$ and then projecting the coefficient to $A_{\Phi}(z)$ we obtain the map

$$
\left[\mathcal{S}^{(a)}\right]: V \rightarrow A_{\Phi}(z), \quad F_{i} \mapsto\left[\frac{a_{i}}{f_{i}}\right]
$$

Theorem 4.12. The composition $\alpha(z) \circ\left[\mathcal{S}^{(a)}\right]: V \rightarrow \operatorname{Sing} V$ is the orthogonal projection multiplied by -1 . The composition $\left[\mathcal{S}^{(a)}\right] \circ \alpha(z): A_{\Phi}(z) \rightarrow A_{\Phi}(z)$ is the identity map multiplied by -1 . 
Proof. The composition $\alpha(z) \circ\left[\mathcal{S}^{(a)}\right]$ sends $F_{i}$ to $v_{i}$ which is the orthogonal projection multiplied by -1 The composition $\left[\mathcal{S}^{(a)}\right] \circ \alpha(z)$ sends $\left[\frac{a_{i}}{f_{i}}\right]$ to

$$
-\left[\frac{a_{i}}{f_{i}}\right]+\frac{a_{i}}{|a|} \sum_{j \in J}\left[\frac{a_{j}}{f_{j}}\right] .
$$

The last sum is zero in $A_{\Phi}(z)$.

\subsection{Multiplication on $\operatorname{Sing} V$ and $(\operatorname{Sing} V)^{*}$.}

Theorem 4.13. The canonical isomorphism $\alpha(z): A_{\Phi}(z) \rightarrow \operatorname{Sing} V$ defines an algebra structure on Sing $V$,

$$
\begin{aligned}
v_{j} *_{z} v_{i} & =\frac{a_{j}}{z_{j}-z_{i}} v_{i}+\frac{a_{i}}{z_{i}-z_{j}} v_{j}, \quad i \neq j, \\
v_{j} *_{z} v_{j} & =-\sum_{i \neq j} v_{j} *_{z} v_{i} .
\end{aligned}
$$

The element

$$
\frac{1}{|a|} \sum_{j \in J} z_{j} v_{j}
$$

is the identity element.

The isomorphism $\left.S^{(a)}\right|_{\operatorname{Sing} V}: \operatorname{Sing} V \rightarrow(\operatorname{Sing} V)^{*}$ induces an algebra structure on $(\operatorname{Sing} V)^{*}$.

Lemma 4.14. The isomorphism $\left.S^{(a)}\right|_{\operatorname{Sing} V}: \operatorname{Sing} V \rightarrow(\operatorname{Sing} V)^{*}$ is given by the formula $v_{j} \mapsto-a_{j} h_{j}$ for all $j$.

Proof. The lemma follows from formulas (4.23) and (4.3).

Corollary 4.15. The multiplication on $(\operatorname{Sing} V)^{*}$ is given by the formula

$$
\begin{aligned}
h_{j} *_{z} h_{i} & =\frac{1}{z_{i}-z_{j}} h_{i}+\frac{1}{z_{j}-z_{i}} h_{j}, \quad i \neq j, \\
a_{j} h_{j} *_{z} h_{j} & =-\sum_{i \neq j} a_{i} h_{i} *_{z} h_{j} .
\end{aligned}
$$

The element

$$
-\frac{1}{|a|} \sum_{j \in J} a_{j} z_{j} h_{j}
$$

is the identity element.

4.8. Tangent morphism. The tangent morphism $\beta$ of the tangent bundle $T\left(\mathbb{C}^{n}-\Delta\right) \rightarrow$ $\mathbb{C}^{n}-\Delta$ to the bundle of algebras $\sqcup_{z \in \mathbb{C}^{n}-\Delta} A_{\Phi}(z) \rightarrow \mathbb{C}^{n}-\Delta$ is given by the formula (3.31),

$$
\beta(z): \partial_{j} \in T_{z}\left(\mathbb{C}^{n}-\Delta\right) \mapsto\left[\frac{\partial \Phi}{\partial z_{j}}\right]=\left[\frac{a_{j}}{z_{j}+t}\right] \in A_{\Phi}(z) .
$$

Lemma 4.16. The map $\beta(z)$ is an epimorphism. The kernel of $\beta(z)$ is generated by the vector $\sum_{j \in J} \partial_{j}$. 
The residue form on the bundle of algebras induces a holomorphic symmetric bilinear form $\eta$ on $T\left(\mathbb{C}^{n}-\Delta\right)$, see formula (3.32). The bilinear form $\eta$ has rank $n-1$. Its kernel is generated by the vector $\sum_{j \in J} \partial_{j}$.

Lemma 4.17. We have

$$
\begin{aligned}
& \eta\left(\partial_{j}, \partial_{j}\right)=-a_{j}+\frac{a_{j}^{2}}{|a|}, \quad j \in J, \\
& \eta\left(\partial_{i}, \partial_{j}\right)=\frac{a_{i} a_{j}}{|a|}, \quad i, j \in J, i \neq j .
\end{aligned}
$$

Proof. The lemma follows from Lemmas 4.1, 4.8 and Theorem 2.7. It can be checked also by a straightforward calculation.

4.9. Multiplication and potential function of second kind. Let us define the multiplication on fibers of $T\left(\mathbb{C}^{n}-\Delta\right)$ by the formulas

$$
\begin{aligned}
\partial_{i} *_{z} \partial_{j} & =\frac{a_{i}}{z_{i}-z_{j}} \partial_{j}+\frac{a_{j}}{z_{j}-z_{i}} \partial_{i}, \\
\partial_{i} *_{z} \partial_{i} & =-\sum_{j \neq i} \partial_{i} *_{z} \partial_{j}
\end{aligned}
$$

cf. formula 5.25 in [D2]. The vector $\sum_{i \in J} \partial_{i}$ has zero product with everything.

Lemma 4.18. For every $z \in \mathbb{C}^{n}-\Delta$, the morphism $\beta(z)$ defines an algebra epimorphism of $T_{z}\left(\mathbb{C}^{n}-\Delta\right)$ to $A_{\Phi}(z)$, in particular, $\beta(z)(v) *_{z} \beta(z)(w)=\beta(z)\left(v *_{z} w\right)$ for all $v, w \in$ $T_{z}\left(\mathbb{C}^{n}-\Delta\right)$.

Consider the ideal of $T_{z}\left(\mathbb{C}^{n}-\Delta\right)$ generated by $\sum_{j \in J} \partial_{j}$. Denote $B(z)$ the quotient algebra. The morphism $\beta(z)$ induces an isomorphism $B(z) \simeq A_{\Phi}(z)$.

Lemma 4.19. The element $\frac{1}{|a|} \sum_{i \in J} z_{j} \partial_{j}$ projects to the identity element of $B(z)$.

The bilinear form $\eta$ defines a morphism $\tilde{\eta}$ of the tangent bundle $T\left(\mathbb{C}^{n}-\Delta\right)$ to the cotangent bundle $T^{*}\left(\mathbb{C}^{n}-\Delta\right)$. For $j \in J$, denote $p_{j}(z)=a_{j} q_{j}(z)=a_{j}\left(-z_{j}+\sum_{i \in J} \frac{a_{i}}{|a|} z_{i}\right)$. We have $\sum_{j \in J} p_{j}=0$.

Lemma 4.20. The morphism $\tilde{\eta}$ is given by the formula $\partial_{j} \mapsto d p_{j}$ for all $j$. The kernel of $\tilde{\eta}$ is generated by the vector $\sum_{j \in J} \partial_{j}$.

Consider the span of differential one-forms $\left(d p_{j}\right)_{j \in J}$. This span equals the span of differential one-forms $\left(d z_{i}-d z_{j}\right)_{1 \leqslant i<j \leqslant n}$. The spans in the fibers define the subbundle

$$
\sqcup_{z \in \mathbb{C}^{n}-\Delta} B^{*}(z) \rightarrow \mathbb{C}^{n}-\Delta
$$

of the cotangent bundle $T^{*}\left(\mathbb{C}^{n}-\Delta\right)$. The subbundle has rank $n-1$.

Lemma 4.21. The form $\eta$ induces the algebra structure on $B^{*}(z)$ given by the formula

$$
\begin{aligned}
d p_{i} *_{z} d p_{j} & =\frac{a_{i}}{z_{i}-z_{j}} d p_{j}+\frac{a_{j}}{z_{j}-z_{i}} d p_{i}=\frac{a_{i} a_{j}}{z_{i}-z_{j}} d\left(z_{i}-z_{j}\right), \quad i \neq j, \\
d p_{i} *_{z} d p_{i} & =-\sum_{j \neq i} d p_{j} *_{z} d p_{i},
\end{aligned}
$$


and the bilinear form

$$
\begin{aligned}
& \left(d p_{j}, d p_{j}\right)=-a_{j}+\frac{a_{j}^{2}}{|a|}, \quad j \in J, \\
& \left(d p_{i}, d p_{j}\right)=\frac{a_{i} a_{j}}{|a|}, \quad i, j \in J, \quad i \neq j .
\end{aligned}
$$

Introduce the potential function of second kind

$$
\tilde{P}(z)=\frac{1}{2} \sum_{1 \leqslant i<j \leqslant n} a_{i} a_{j}\left(z_{i}-z_{j}\right)^{2} \log \left(z_{i}-z_{j}\right) .
$$

Theorem 4.22. We have

$$
d\left(\frac{\partial^{2} \tilde{P}}{\partial z_{i} \partial z_{j}}\right)=-\tilde{\eta}\left(\partial_{i}\right) *_{z} \tilde{\eta}\left(\partial_{j}\right)
$$

for all $i, j$.

Proof. The theorem follows from Lemma 4.21.

Notice that equation (4.37) is the definition (3.5) in [D2] of the potential function of an almost dual Frobenius structure.

The right hand side in (4.37) can be rewritten: $\tilde{\eta}\left(\partial_{i}\right) *_{z} \tilde{\eta}\left(\partial_{j}\right)=\tilde{\eta}\left(\partial_{i} *_{z} \partial_{j}\right)$. For all $i, j, k$, we have

$$
\tilde{\eta}\left(\partial_{i} *_{z} \partial_{j}\right)\left(\partial_{\ell}\right)=\eta\left(\partial_{i} *_{z} \partial_{j}, \partial_{\ell}\right)=\left(\beta(z)\left(\partial_{i}\right) *_{z} \beta(z)\left(\partial_{j}\right) *_{z} \beta(z)\left(\partial_{\ell}\right),[1](z)\right)_{z}
$$

where $(,)_{z}$ is the residue form on $A_{\Phi}(z)$. Formula (4.37) says that for all $i, j, k$, we have

$$
\frac{\partial^{3} \tilde{P}}{\partial z_{i} \partial z_{j} \partial_{\ell}}(z)=-\left(\beta(z)\left(\partial_{i}\right) *_{z} \beta(z)\left(\partial_{j}\right) *_{z} \beta(z)\left(\partial_{\ell}\right),[1](z)\right)_{z} .
$$

Hence Conjecture 3.14 holds for this family of arrangements.

4.10. Connections on the bundle $\sqcup_{z \in \mathbb{C}^{n}-\Delta} B^{*}(z) \rightarrow \mathbb{C}^{n}-\Delta$ defined in (4.33). The combinatorial and Gauss-Manin connections on $\left(\mathbb{C}^{n}-\Delta\right) \times \operatorname{Sing} V \rightarrow \mathbb{C}^{n}-\Delta$ induce the combinatorial and Gauss-Manin connections on bundle (4.33).

Lemma 4.23. The differential one-forms $\left(d p_{j}\right)_{j \in J}$ are flat sections of the combinatorial connection on bundle (4.33).

Proof. The vectors $v_{j} \in \operatorname{Sing} V$ give flat sections of the combinatorial connection on $\mathbb{C}^{n} \times$ Sing $V \rightarrow \mathbb{C}^{n}$. By formula (4.19), the elements $\left[\frac{a_{j}}{z_{j}+t}\right] \in A_{\Phi}(z)$ give flat sections of bundle of algebras. Now formula (4.30) and Lemma 4.20 imply Lemma 4.23.

Let $I(z)=\sum_{j \in J} I^{j}(z) d p_{j}$ be a section of bundle (4.33). For $i \in J$, we denote $\frac{\partial I}{\partial z_{i}}=$ $\sum \frac{\partial I^{j}}{\partial z_{i}} d p_{j}$ 
Lemma 4.24. The differential equations for flat sections of the Gauss-Manin connection take the from

$$
\kappa \frac{\partial I}{\partial z_{i}}=d p_{i} *_{z} I, \quad i \in J
$$

see formula (4.34). For generic $\kappa$ all the flat sections are given by the formula

$$
I_{\gamma, \kappa}(z)=\sum_{j \in J}\left(\int_{\gamma(z)} \prod_{i \in J}\left(z_{i}+t\right)^{a_{i} / \kappa} \frac{d t}{z_{j}+t}\right) d p_{j},
$$

where $\gamma(z) \in H_{1}\left(U(\mathcal{C}(z)),\left.\mathcal{L}_{\kappa}\right|_{U(\mathcal{C}(z))}\right)$ is a flat section of the Gauss-Manin connection on $\sqcup_{z \in \mathbb{C}^{n}-\Delta} H_{k}\left(U(\mathcal{C}(z)),\left.\mathcal{L}_{\kappa}\right|_{U(\mathcal{C}(z))}\right) \rightarrow \mathbb{C}^{n}-\Delta$.

Proof. The lemma follows from Theorem 3.3 and formula (4.9).

Theorem 4.25. For every flat section $I_{\gamma, \kappa}$, we have $I_{\gamma, \kappa}=-\kappa d p_{\gamma, \kappa}$ where

$$
p_{\gamma, \kappa}=\int_{\gamma(z)} \prod_{i \in J}\left(z_{i}+t\right)^{a_{i} / \kappa} d t
$$

Proof. The theorem follows from two formulas:

$$
\kappa \frac{\partial p_{\gamma, \kappa}}{\partial z_{j}}=\int_{\gamma(z)} \prod_{i \in J}\left(z_{i}+t\right)^{a_{i} / \kappa} \frac{a_{j} d t}{z_{j}+t}
$$

and $\sum_{j \in J} \frac{\partial p_{\gamma, \kappa}}{\partial z_{j}}=0$.

Following Dubrovin [D1, D2], we will call the functions $p_{\gamma, \kappa}$ twisted periods. Notice that this definition agrees with the definition of twisted periods in Section 3.11, namely, the twisted periods of Theorem 4.25 can be also defined by formula (3.42) of Theorem 3.13.

Lemma 4.26. Given $\kappa \in \mathbb{C}^{\times}$, let $I(z, \kappa)$ be a flat section of the Gauss-Manin connection with the parameter $\kappa$. Let $I(z,-\kappa)$ be a flat section of the Gauss-Manin connection with the parameter $-\kappa$. Then $(I(z, \kappa), I(z,-\kappa))_{z}$ does not depend on $z \in \mathbb{C}^{n}-\Delta$.

Proof. The lemma follows from the fact that $B^{*}(z)$ is a Frobenius algebra.

4.11. Functoriality. In this section we will discuss how our objects extend to strata of the discriminant $\Delta \subset \mathbb{C}^{n}$.

4.11.1. A stratum $X$ of $\Delta$ is given by a partition $\left(J_{1}, \ldots, J_{m}\right)$ of $J$,

$$
X=\left\{\left(z_{1}, \ldots, z_{n}\right) \in \mathbb{C}^{n} \mid z_{i}-z_{j}=0 \quad \text { for } i, j \in J_{\ell}, \ell=1, \ldots, m\right\},
$$

$\operatorname{dim} X=m$. The coordinates on $X$ are functions $x_{1}, \ldots, x_{m}$ where $x_{\ell}=z_{j}$ for $j \in J_{\ell}$. Let $\iota: X \hookrightarrow \mathbb{C}^{n}$ be the natural embedding. Then

$$
\iota_{*}: \frac{\partial}{\partial x_{\ell}} \mapsto \sum_{j \in J_{\ell}} \frac{\partial}{\partial z_{j}}, \quad \iota^{*}: d z_{j} \mapsto d x_{\ell} \quad \text { if } j \in J_{\ell} .
$$

The remaining strata of $\Delta$ cut on $X$ the union of hyperplanes $x_{i}=x_{j}, 1 \leqslant i<j \leqslant m$ which we denote $\Delta_{X}$. For $\ell=1, \ldots, m$, we denote $b_{\ell}=\sum_{j \in J_{\ell}} a_{j}$. We assume that $b_{\ell} \neq 0$ for all $\ell$.

We restrict our family of arrangements $\mathcal{C}(z), z \in \mathbb{C}^{n}$, to $X-\Delta_{X}$. For $x \in X-\Delta_{X}$ the corresponding arrangement $\mathcal{C}(x)$ consists of points $-x_{1}, \ldots,-x_{m}$ of weights $b_{1}, \ldots, b_{m}$, 
respectively. For this new family we will construct all the objects described in Sections 4.1 4.10 and relate them to the objects constructed for the arrangements $\mathcal{C}(z), z \in \mathbb{C}^{n}-\Delta$. The objects corresponding to the new family will be provided with the index $X$.

4.11.2. For $x \in X-\Delta_{X}$, the space $\left(V_{X}\right)^{*}=\mathcal{A}^{1}(\mathcal{C}(x))$ has the standard basis $\left(H_{\ell, X}\right), \ell=$ $1, \ldots, m$. Recall that the space $V^{*}$ of Sections $4.1+4.10$ has the standard basis $\left(H_{j}\right), j \in J$. We have the canonical epimorphism

$$
f^{*}: V^{*} \rightarrow\left(V_{X}\right)^{*}, \quad\left(H_{j}\right) \mapsto\left(H_{\ell, X}\right) \quad \text { if } \quad j \in J_{\ell} .
$$

The space $V_{X}=\mathcal{F}^{1}(\mathcal{C}(x))$ has the standard basis $F_{\ell, X}, \ell=1, \ldots, m$. The space $V$ of Sections 4.1-4.10 has the standard basis $F_{j}, j \in J$. We have the canonical embedding

$$
f: V_{X} \hookrightarrow V, \quad F_{\ell, X} \mapsto \sum_{j \in J_{\ell}} F_{j} .
$$

The subspace of singular vector is defined by the formula

$$
\text { Sing } V_{X}=\left\{\sum_{\ell=1}^{m} c_{\ell} F_{\ell, X} \mid \sum_{\ell=1}^{m} b_{\ell} c_{\ell}=0\right\} .
$$

We have $f\left(\operatorname{Sing} V_{X}\right)=f\left(V_{X}\right) \cap(\operatorname{Sing} V)$. Consider the embedding

$$
\tilde{f} \text { : Sing } V_{X} \hookrightarrow \operatorname{Sing} V, \quad v \mapsto f(v) .
$$

For the contravariant form on $V_{X}$ we have

$$
S_{X}^{(b)}\left(F_{\ell, X}, F_{k, X}\right)=S^{(a)}\left(f\left(F_{\ell, X}\right), f\left(F_{k, X}\right)\right)=\delta_{\ell, k} b_{k} .
$$

For $\ell=1, \ldots, m$, we define a vector $v_{\ell, X} \in \operatorname{Sing} V_{X}$ by the formula

$$
v_{\ell, X}=-F_{\ell, X}+\frac{b_{\ell}}{|a|} \sum_{k=1}^{m} F_{k, X}
$$

We have $f: v_{\ell, X} \mapsto \sum_{j \in J_{\ell}} v_{j}$.

The standard basis $\left(H_{\ell, X}\right), \ell=1, \ldots, m$, induces linear functions on $\operatorname{Sing} V_{X}$,

$$
h_{\ell, X}: v_{k, X} \mapsto \frac{b_{k}}{|a|} \quad \text { if } \quad k \neq \ell, \quad v_{\ell, X} \mapsto-1+\frac{b_{\ell}}{|a|} .
$$

We have $\tilde{f}^{*}: h_{j} \mapsto h_{\ell, X}$ if $j \in J_{\ell}$.

4.11.3. For $\ell=1, \ldots, m$ and $x \in X-\Delta_{X}$, the operators $K_{\ell, X}(x): \operatorname{Sing} V_{X} \rightarrow \operatorname{Sing} V_{X}$ are defined by formulas (4.7),

$$
\begin{aligned}
K_{\ell, X}(x) v_{k, X} & =\frac{b_{\ell}}{x_{\ell}-x_{k}} v_{k, X}+\frac{b_{k}}{x_{k}-x_{\ell}} v_{\ell, X} \quad \text { for } \quad \ell \neq k, \\
K_{\ell, X}(x) v_{\ell, X} & =-\sum_{k \neq \ell} K_{\ell, X}(x) v_{k, X} .
\end{aligned}
$$

For all $\ell, k$, we have

$$
f\left(K_{\ell, X}(x) v_{k, X}\right)=\sum_{j \in J_{\ell}} K_{j}(x) f\left(v_{k, X}\right) .
$$


Notice that the right hand side in (4.54) is well-defined despite the fact that $K_{j}(x) v_{i}$ is not well-defined for all $v_{i}$, see formula (4.7).

4.11.4. Multiplication on $\operatorname{Sing} V_{X}$ is defined by formulas (4.26),

$$
\begin{aligned}
v_{\ell, X} *_{x, X} v_{k, X} & =\frac{b_{\ell}}{x_{\ell}-x_{k}} v_{k, X}+\frac{b_{k}}{x_{k}-x_{\ell}} v_{\ell, X} \quad \text { for } \quad \ell \neq k, \\
v_{\ell, X} *_{x, X} v_{\ell, X} & =-\sum_{k \neq \ell} v_{\ell, X} *_{x, X} v_{k, X} .
\end{aligned}
$$

For all $\ell, k$, we have

$$
f\left(v_{\ell, X} *_{x, X} v_{k, X}\right)=f\left(v_{\ell, X}\right) *_{x} f\left(v_{k, X}\right) .
$$

Notice that the right hand side in (4.56) is well-defined despite the fact that $v_{i} *_{x} v_{j}$ is not well-defined for all $v_{i}, v_{j}$, see formula (4.26).

4.11.5. The multiplication on $\left(\operatorname{Sing} V_{X}\right)^{*}$ is given by formula (4.28),

$$
\begin{aligned}
h_{\ell, X} *_{x, X} h_{k, X} & =\frac{1}{x_{\ell}-x_{k}} h_{\ell, X}+\frac{1}{z_{k}-z_{\ell}} h_{k, X}, \quad \ell \neq k, \\
b_{\ell} h_{\ell, X} *_{x, X} h_{k, \ell} & =-\sum_{k \neq \ell} b_{k} h_{k, X} *_{x, X} h_{\ell, X} .
\end{aligned}
$$

If $\ell \neq k, i \in J_{\ell}, j \in J_{k}$, then

$$
\tilde{f}^{*}\left(h_{i} *_{x} h_{j}\right)=h_{\ell, X} *_{x, X} h_{k, X} .
$$

Notice that $h_{i} *_{x} h_{j}$ is well-defined despite the fact that $h_{i} *_{x} h_{j}$ is not well-defined for all $h_{i}, h_{j}$, see formula (4.26).

4.11.6. For $x \in X-\Delta_{X}$ the residue form on $A_{\Phi}(x)$ induces a holomorphic bilinear form $\eta_{X}$ on $T_{x}\left(X-\Delta_{X}\right)$,

$$
\begin{aligned}
& \eta_{X}\left(\frac{\partial}{\partial x_{\ell}}, \frac{\partial}{\partial x_{\ell}}\right)=-b_{\ell}+\frac{b_{\ell}^{2}}{|a|}, \quad j \in J, \\
& \eta_{X}\left(\frac{\partial}{\partial x_{\ell}}, \frac{\partial}{\partial x_{k}}\right)=\frac{b_{\ell} b_{k}}{|a|}, \quad \ell \neq k .
\end{aligned}
$$

For all $\ell, k$, have

$$
\eta_{X}\left(\frac{\partial}{\partial x_{\ell}}, \frac{\partial}{\partial x_{k}}\right)=\eta\left(\sum_{i \in J_{\ell}} \frac{\partial}{\partial z_{i}}, \sum_{j \in J_{\ell}} \frac{\partial}{\partial z_{j}}\right) .
$$

For $\ell=1, \ldots, m$, we define a linear function on $X$,

$$
q_{\ell, X}(x)=-x_{\ell}+\sum_{k \neq \ell} \frac{b_{k}}{|a|} x_{k} .
$$

We have

$$
q_{\ell, X}(x)=q_{i}(x), \quad \text { if } i \in J_{\ell} .
$$


The period map $q_{X}: X-\Delta_{X} \rightarrow \operatorname{Sing} V_{X}$ is defined by formula (4.21),

$$
q_{X}(x)=\frac{1}{|a|} \sum_{\ell=1}^{m} q_{\ell, X}(x) F_{\ell, X}=\frac{1}{|a|} \sum_{\ell=1}^{m} x_{\ell} v_{\ell, X} .
$$

Theorem 4.27. For all $x \in X$, we have

$$
f\left(q_{X}(x)\right)=q(x)
$$

and for the potential functions of first kind we have

$$
P_{X}(x)=P(x) \text {. }
$$

4.11.7. For $x \in X-\Delta_{X}$, the multiplication on $T_{x}\left(X-\Delta_{X}\right)$ is defined by formulas (4.32),

$$
\begin{aligned}
\frac{\partial}{\partial x_{\ell}} *_{x, X} \frac{\partial}{\partial x_{k}} & =\frac{b_{\ell}}{x_{\ell}-x_{k}} \frac{\partial}{\partial x_{k}}+\frac{b_{k}}{x_{k}-x_{\ell}} \frac{\partial}{\partial x_{\ell}}, \\
\frac{\partial}{\partial x_{\ell}} *_{x, X} \frac{\partial}{\partial x_{\ell}} & =-\sum_{k \neq \ell} \frac{\partial}{\partial x_{k}} *_{x, X} \frac{\partial}{\partial x_{\ell}} .
\end{aligned}
$$

For all $\ell, k$, we have

$$
\frac{\partial}{\partial x_{\ell}} *_{x, X} \frac{\partial}{\partial x_{k}}=\left(\sum_{i \in J_{\ell}} \frac{\partial}{\partial z_{i}}\right) *_{x}\left(\sum_{j \in J_{k}} \frac{\partial}{\partial z_{j}}\right) .
$$

Notice that the right hand side in (4.67) is well-defined despite the fact that $\frac{\partial}{\partial z_{i}} *_{x} \frac{\partial}{\partial z_{j}}$ is not well-defined for all $i, j$, see (4.66).

4.11.8. For $\ell=1, \ldots, m$, we denote $p_{\ell, X}=b_{\ell} q_{\ell, X}$. The map

$$
\tilde{\eta}_{X}: T_{x}\left(X-\Delta_{X}\right) \rightarrow T_{x}^{*}\left(X-\Delta_{X}\right), \quad w \mapsto \eta_{X}(w, \cdot),
$$

sends $\frac{\partial}{\partial x_{\ell}}$ to $d p_{\ell, X}$. We have

$$
d p_{\ell, X}=\iota^{*}\left(\sum_{i \in J_{\ell}} d p_{i}\right)
$$

Denote $B_{X}^{*}(x)$ the span of $\left(d p_{\ell, X}\right)_{\ell=1}^{m}$ in $T_{x}^{*}\left(X-\Delta_{X}\right)$. This span equals the span of differential forms $\left(d x_{\ell}-d x_{k}\right)_{1 \leqslant \ell<k \leqslant m}$. The multiplication on $B_{X}^{*}(x)$ is given by the formulas (4.34),

$$
\begin{aligned}
d p_{\ell, X} *_{x, X} d p_{k, X} & =\frac{b_{\ell}}{x_{\ell}-x_{k}} d p_{k, X}+\frac{b_{k}}{x_{k}-x_{\ell}} d p_{\ell, X}=\frac{b_{\ell} b_{k}}{x_{\ell}-x_{k}} d\left(x_{\ell}-x_{k}\right), \quad \ell \neq k \\
d p_{\ell, X} *_{x, X} d p_{\ell, X} & =-\sum_{k \neq \ell} d p_{k, X} *_{x, X} d p_{\ell, X} .
\end{aligned}
$$

For all $\ell, k$, we have

$$
\iota^{*}\left(\sum_{i \in J_{\ell}} d p_{i}\right) *_{x, X} \iota^{*}\left(\sum_{j \in J_{k}} d p_{j}\right)=\iota^{*}\left(\sum_{i \in J_{\ell}} \sum_{j \in J_{k}} d p_{i} *_{x} d p_{j}\right) .
$$


The potential function of second kind is defined by formula (4.36),

$$
\tilde{P}_{X}\left(x_{1}, \ldots, x_{m}\right)=\frac{1}{2} \sum_{1 \leqslant \ell<k \leqslant m} b_{\ell} b_{k}\left(x_{\ell}-x_{k}\right)^{2} \log \left(x_{\ell}-x_{k}\right) .
$$

By formula (4.37),

$$
d\left(\frac{\partial^{2} \tilde{P}_{X}}{\partial x_{\ell} \partial x_{k}}\right)=-\tilde{\eta}_{X}\left(\frac{\partial}{\partial x_{\ell}}\right) *_{x, X} \tilde{\eta}_{X}\left(\frac{\partial}{\partial x_{k}}\right)
$$

for all $\ell, k$. For all $\ell, k$, we have

$$
\frac{\partial^{2} \tilde{P}_{X}}{\partial x_{\ell} \partial x_{k}}(x)=\lim _{\substack{z \rightarrow x \\ z \in \mathbb{C}^{n}-\Delta}} \sum_{i \in J_{\ell}} \sum_{j \in J_{k}} \frac{\partial^{2} \tilde{P}}{\partial z_{i} \partial z_{j}}(z) .
$$

4.12. Frobenius like structure. Consider the quotient $M$ of $\mathbb{C}^{n}$ by the one-dimensional subspace $\mathbb{C}(1, \ldots, 1)$ and the natural projection $\pi: \mathbb{C}^{n} \rightarrow M$. Then all our objects the combinatorial bundle $\left(\mathbb{C}^{n}-\Delta\right) \times(\operatorname{Sing} V) \rightarrow \mathbb{C}^{n}-\Delta$ with the contravariant form $S^{(a)}$ and connections (combinatorial and Gauss-Manin); the bundle of algebras $\sqcup_{z \in \mathbb{C}^{n}-\Delta} A_{\Phi}(z) \rightarrow$ $\mathbb{C}^{n}-\Delta$; the period map $q: \mathbb{C}^{n}-\Delta \rightarrow \operatorname{Sing} V$, potential functions $P(z)$ and $\tilde{P}(z)$, flat periods $p_{j}(z)$, twisted periods $p_{\gamma, \kappa}(z)$ - descend to the quotient and form on $M-\pi(\Delta)$ a structure which we will also call a Forbenius like structure.

In particular, the functions $p_{1}, \ldots, p_{n-1}$ will form a coordinate systems on $M$ and $\eta$ will induce a holomorphic metric on $M$ constant with respect to the coordinates $p_{1}, \ldots, p_{n-1}$. If $y_{i}=\sum_{j=1}^{n-1} c_{j, i} p_{j}, j=1, \ldots, n-1$, is a linear change of coordinates with $c_{i, j} \in \mathbb{C}$ such that $\eta=\sum_{j=1}^{n-1} d y_{j}^{2}$. Then equation (4.37) will take the form

$$
d\left(\frac{\partial^{2} \tilde{P}}{\partial y_{i} \partial y_{j}}\right)=-d y_{i} *_{y} d y_{j}
$$

for all $i, j$. The functions $-\frac{\partial^{3} \tilde{P}}{\partial y_{i} \partial y_{j} \partial y_{k}}$ will become the structure constants of the multiplication on $T_{y}^{*}(M-\pi(\Delta))$ and the potential function $\tilde{P}$ will satisfies the WDVV equations with respect to the coordinates $y_{1}, \ldots, y_{n-1}$.

\section{GENERIC Lines ON PLANE}

5.1. An arrangement in $\mathbb{C}^{n} \times \mathbb{C}^{2}$. Consider $\mathbb{C}^{2}$ with coordinates $t_{1}, t_{2}, \mathbb{C}^{n}$ with coordinates $z_{1}, \ldots, z_{n}$. Fix $n$ linear functions on $\mathbb{C}^{2}, g_{j}=b_{j}^{1} t_{1}+b_{j}^{2} t_{2}, j \in J, b_{j}^{i} \in \mathbb{C}$. We assume that

$$
d_{i, j}=\operatorname{det}\left(\begin{array}{cc}
b_{i}^{1} & b_{i}^{2} \\
b_{j}^{1} & b_{j}^{2}
\end{array}\right) \neq 0 \quad \text { for all } i \neq j .
$$

We define $n$ linear functions on $\mathbb{C}^{n} \times \mathbb{C}^{2}, f_{j}=z_{j}+g_{j}, j \in J$. In $\mathbb{C}^{n} \times \mathbb{C}^{2}$ we define the arrangement $\tilde{\mathcal{C}}=\left\{\tilde{H}_{j} \mid f_{j}=0, j \in J\right\}$.

For every $z=\left(z_{1}, \ldots, z_{n}\right)$ the arrangement $\tilde{\mathcal{C}}$ induces an arrangement $\mathcal{C}(z)$ in the fiber of the projection $\mathbb{C}^{n} \times \mathbb{C}^{2} \rightarrow \mathbb{C}^{n}$ over $z$. We identify every fiber with $\mathbb{C}^{2}$. Then $\mathcal{C}(z)$ consists of lines $H_{j}(z), j \in J$, defined in $\mathbb{C}^{2}$ by the equations $f_{j}=0$. Denote $U(\mathcal{C}(z))=\mathbb{C}^{2}-\cup_{j \in J} H_{j}(z)$, the complement to the arrangement $\mathcal{C}(z)$. 
The arrangement $\mathcal{C}(z)$ is with normal crossings if and only if $z \in \mathbb{C}^{n}-\Delta$, where $\Delta=$ $\cup_{1 \leqslant i<j<k \leqslant n} H_{i, j, k}$ and the hyperplane $H_{i, j, k}$ is defined by the equation $f_{i, j, k}=0$,

$$
f_{i, j, k}=d_{j, k} z_{i}+d_{k, i} z_{j}+d_{i, j} z_{k} .
$$

Lemma 5.1. For any four distinct indices $i, j, k, \ell$ we have

$$
\begin{gathered}
\frac{f_{i, j, k}}{d_{k, i} d_{i, j}}+\frac{f_{i, k, \ell}}{d_{\ell, i} d_{i, k}}+\frac{f_{i, \ell, j}}{d_{j, i} d_{i, \ell}}=0 \\
\frac{f_{i, j, k}^{2}}{d_{i, j} d_{j, k} d_{k, i}}-\frac{f_{j, k, \ell}^{2}}{d_{j, k} d_{k, \ell} d_{\ell, j}}+\frac{f_{k, \ell, i}^{2}}{d_{k, \ell} d_{\ell, i} d_{i, k}}-\frac{f_{\ell, i, j}^{2}}{d_{\ell, i} d_{i, j} d_{j, \ell}}=0 .
\end{gathered}
$$

5.2. Good fibers. For any $z \in \mathbb{C}^{n}-\Delta$, the space $\mathcal{A}^{2}(\mathcal{C}(z))$ has the standard basis $\left(H_{i}(z), H_{j}(z)\right), 1 \leqslant i<j \leqslant n$. The space $\mathcal{F}^{2}(\mathcal{C}(z))$ has the standard dual basis $F\left(H_{i}(z), H_{j}(z)\right)$, $1 \leqslant i<j \leqslant n$. For $z^{1}, z^{2} \in \mathbb{C}^{n}-\Delta$, the combinatorial connection identifies the spaces $\mathcal{A}^{2}\left(\mathcal{C}\left(z^{1}\right)\right), \mathcal{F}^{2}\left(\mathcal{C}\left(z^{1}\right)\right)$ with the spaces $\mathcal{A}^{2}\left(\mathcal{C}\left(z^{2}\right)\right), \mathcal{F}^{2}\left(\mathcal{C}\left(z^{2}\right)\right)$, respectively, by identifying the standard bases.

Assume that nonzero weights $\left(a_{j}\right)_{j \in J}$ are given. Then the arrangement $\mathcal{C}(z)$ is weighted. For $z \in \mathbb{C}^{n}-\Delta$, the arrangement $\mathcal{C}(z)$ is unbalanced if $|a| \neq 0$. We assume that $|a| \neq 0$.

For $z \in \mathbb{C}^{n}-\Delta$, we denote $V=\mathcal{F}^{2}(\mathcal{C}(z)), V^{*}=\left(\mathcal{F}^{2}(\mathcal{C}(z))^{*}=\mathcal{A}^{2}(\mathcal{C}(z)), F_{i, j}=\right.$ $F\left(H_{i}(z), H_{j}(z)\right)$. We have $F_{i, j}=-F_{j, i}$,

$$
\begin{gathered}
S^{(a)}\left(F_{i, j}, F_{k, \ell}\right)=0, \quad \text { if } i<j, k<\ell \text { and }(i, j) \neq(k, \ell), \\
S^{(a)}\left(F_{i, j}, F_{i, j}\right)=a_{i} a_{j}, \\
\text { Sing } V=\left\{\sum_{1 \leqslant i<j \leqslant n} c_{i, j} F_{i, j} \mid \sum_{j=1}^{i-1} a_{j} c_{j, i}-\sum_{j=i+1}^{n} a_{j} c_{i, j}=0, i=1, \ldots, n\right\} .
\end{gathered}
$$

By Corollary 2.8, the restriction of $S^{(a)}$ to $\operatorname{Sing} V$ is nondegenerate. Denote $(\operatorname{Sing} V)^{\perp}$ the orthogonal complement to $\operatorname{Sing} V$ with respect to $S^{(a)}$. Then $V=\operatorname{Sing} V \oplus(\operatorname{Sing} V)^{\perp}$. Denote $\pi: V \rightarrow \operatorname{Sing} V$ the orthogonal projection.

Lemma 5.2. The space $(\operatorname{Sing} V)^{\perp}$ is generated by vectors

$$
\sum_{i \in J} F_{i, j}, \quad j \in J
$$

For $i \neq j$, we define the vector $v_{i, j} \in V$ by the formula

$$
v_{i, j}=F_{i, j}-\frac{a_{j}}{|a|} \sum_{k \in J} F_{i, k}-\frac{a_{i}}{|a|} \sum_{\ell \in J} F_{\ell, j} .
$$

We have $v_{i, j}=-v_{j, i}$. Set $v_{i, i}=0$.

Lemma 5.3. We have the following properties.

(i) $\operatorname{dim} \operatorname{Sing} V=\left(\begin{array}{c}n-1 \\ 2\end{array}\right)$.

(ii) We have $v_{i, j} \in \operatorname{Sing} V$ and $v_{i, j}=\pi\left(F_{i, j}\right)$. 
(iii) For $j \in J$, we have $\sum_{i \in J} v_{i, j}=0$.

(iv) For any $k \in J$, the set $v_{i, j}, 1 \leqslant i<j \leqslant n, k \notin\{i, j\}$, is a basis of $\operatorname{Sing} V$.

Lemma 5.4. We have

$$
\begin{aligned}
& S^{(a)}\left(v_{i, j}, v_{k, \ell}\right)=0, \quad \text { if } i, j, k, \ell \text { are distinct, } \\
& S^{(a)}\left(v_{i, j}, v_{i, k}\right)=-\frac{a_{i} a_{j} a_{k}}{|a|}, \quad \text { if } i, j, k \text { are distinct, } \\
& S^{(a)}\left(v_{i, j}, v_{i, j}\right)=-\sum_{k \neq j} S^{(a)}\left(v_{i, j}, v_{i, k}\right)=a_{i} a_{j}-\frac{a_{i} a_{j}\left(a_{i}+a_{j}\right)}{|a|} .
\end{aligned}
$$

5.3. Operators $K_{i}(z): V \rightarrow V$. For any subset $\{i, j, k\} \subset J$, we define the linear operator $L_{i, j, k}: V \rightarrow V$ by the formula

$$
\begin{aligned}
F_{i, j} & \mapsto a_{k} F_{i, j}+a_{i} F_{j, k}+a_{j} F_{k, i}, \\
F_{j, k} & \mapsto a_{k} F_{i, j}+a_{i} F_{j, k}+a_{j} F_{k, i}, \\
F_{k, i} & \mapsto a_{k} F_{i, j}+a_{i} F_{j, k}+a_{j} F_{k, i}, \\
F_{\ell, m} & \mapsto 0, \quad \text { if }\{\ell, m\} \text { is not a subset of }\{i, j, k\} .
\end{aligned}
$$

see formula (3.5). Notice that $L_{i, j, k}$ does not depend on the order of $i, j, k$.

We define the operators $K_{i}(z): V \rightarrow V, i \in J$, by the formula

$$
K_{i}(z)=\sum \frac{d_{j, k}}{f_{i, j, k}} L_{i, j, k}
$$

where the sum is over all unordered subsets $\{j, k\} \subset J-\{i\}$, see formula (3.6). For any $i \in J$ and $z \in \mathbb{C}^{n}-\Delta$, the operator $K_{i}(z)$ preserves the subspace Sing $V \subset V$ and is a symmetric operator, $S^{(a)}\left(K_{i}(z) v, w\right)=S^{(a)}\left(v, K_{i}(z) w\right)$ for all $v, w \in V$, see Theorem 3.2 .

Lemma 5.5. For $i \in J$, we have

$$
\begin{aligned}
K_{i}(z) v_{j, k} & =\frac{d_{j, k}}{f_{i, j, k}}\left(a_{i} v_{j, k}+a_{j} v_{k, i}+a_{k} v_{i, j}\right), \quad \text { if } i \notin\{j, k\}, \\
K_{i}(z) v_{j, i} & =-\sum_{k \notin\{i, j\}} K_{i}(z) v_{j, k} .
\end{aligned}
$$

Proof. The restriction of $K_{i}(z)$ to $(\operatorname{Sing} V)^{\perp}$ is zero by formula (5.10) and Lemma 5.2. We have

$$
K_{i}(z) v_{j, k}=K_{i}(z) F_{j, k}=\frac{d_{j, k}}{f_{i, j, k}}\left(a_{i} F_{j, k}+a_{j} F_{k, i}+a_{k} F_{i, j}\right) .
$$

The right hand side in (5.14) equals the right hand side in (5.12) by Lemma 5.3,

The differential equations (3.14) for flat sections of the Gauss-Manin connection on $\left(\mathbb{C}^{n}-\Delta\right) \times \operatorname{Sing} V \rightarrow \mathbb{C}^{n}-\Delta$ take the form

$$
\kappa \frac{\partial I}{\partial z_{j}}(z)=K_{j}(z) I(z), \quad j \in J
$$


For generic $\kappa$ all the flat sections are given by the formula

$$
I_{\gamma}(z)=\sum_{1 \leqslant i<j \leqslant n}\left(\int_{\gamma(z)} \prod_{m \in J} f_{m}^{a_{m} / \kappa} \frac{d_{i, j}}{f_{i} f_{j}} d t_{1} \wedge d t_{2}\right) F_{i, j},
$$

see formula (3.12). These generic $\kappa$ can be determined more precisely from the determinant formula in [V1].

5.4. Conformal blocks. Define the map $q: \mathbb{C}^{n} \rightarrow \operatorname{Sing} V$ by the formula

$$
q: z \mapsto-\frac{1}{|a|^{2}} \sum_{1 \leqslant i<j \leqslant n} \frac{\left(z_{i} b_{j}^{1}-z_{j} b_{i}^{1}\right)^{2}}{d_{i, j} b_{i}^{1} b_{j}^{1}} v_{i, j} .
$$

Lemma 5.6. For any $k \in J$, we have

$$
q(z)=\frac{1}{|a|^{2}} \sum^{\prime} \frac{f_{i, j, k}^{2}}{d_{i, j} d_{j, k} d_{k, i}} v_{i, j}
$$

where the sum is over all pairs $i<j$ such that $k \notin\{i, j\}$.

Proof. Denote $A_{i, j}=-\frac{\left(z_{i} b_{j}^{1}-z_{j} b_{i}^{1}\right)^{2}}{d_{i, j} b_{i}^{1} b_{j}^{1}}$, then

$$
q(z)=\frac{1}{|a|^{2}} \sum_{1 \leqslant i<j \leqslant n} A_{i, j} v_{i, j} .
$$

We replace in (5.19) each $v_{i, k}$ with $-\sum_{j \neq k} v_{i, j}$ and each $v_{k, j}$ with $-\sum_{i \neq k} v_{i, j}$. Then

$$
q(z)=\frac{1}{|a|^{2}} \sum^{\prime}\left(A_{i, j}+A_{j, k}+A_{k, i}\right) v_{i, j}
$$

where the sum is the same as in (5.18). The lemma follows from the identity

$$
A_{i, j}+A_{j, k}+A_{k, i}=\frac{f_{i, j, k}^{2}}{d_{i, j} d_{j, k} d_{k, i}} .
$$

By Lemma 5.6, the map $q$ can be defined in terms of the determinants $d_{i, j}, 1 \leqslant i<j \leqslant n$, only without using the individual numbers $b_{i}^{1}$.

Theorem 5.7. If $\kappa=|a| / 2$, then the Gauss-Manin connection on $\left(\mathbb{C}^{n}-\Delta\right) \times(\operatorname{Sing} V) \rightarrow$ $\mathbb{C}^{n}-\Delta$ has a one-dimensional invariant subbundle, generated by the section $q: z \mapsto q(z)$, see (5.18). This section is flat.

This one-dimensional subbundle will be called the bundle of conformal blocks at level $|a| / 2$. Proof. We check that

$$
\frac{|a|}{2} \frac{\partial q}{\partial z_{1}}(z)=K_{1}(z) q(z)
$$

The other differential equations are proved similarly. By Lemma 5.6 we have

$$
q(z)=\frac{1}{|a|^{2}} \sum_{1<i<j \leqslant n} \frac{f_{1, i, j}^{2}}{d_{i, j} d_{j, 1} d_{1, i}} v_{i, j} .
$$


Then

$$
\frac{|a|}{2} \frac{\partial q}{\partial z_{1}}(z)=\frac{1}{|a|} \sum_{1<i<j \leqslant n} \frac{f_{1, i, j}}{d_{j, 1} d_{1, i}} v_{i, j}
$$

and

$$
K_{1}(z) q(z)=\frac{1}{|a|^{2}} \sum_{1<i<j \leqslant n} \frac{f_{1, i, j}}{d_{j, 1} d_{1, i}}\left(a_{1} v_{i, j}+a_{i} v_{j, 1}+a_{j} v_{1, i}\right)
$$

by formula (5.12). By replacing $v_{j, 1}$ with $-\sum_{i \neq 1} v_{j, i}$ and $v_{1, i}$ with $-\sum_{j \neq 1} v_{i, j}$ and using Lemma 5.1 we obtain

$$
K_{1}(z) q(z)=\frac{1}{|a|} \sum_{1<i<j \leqslant n} \frac{f_{1, i, j}}{d_{j, 1} d_{1, i}} v_{i, j}
$$

5.5. Algebra $A_{\Phi}(z)$. The master function of the arrangement $\mathcal{C}(z)$ is

$$
\Phi(z, t)=\sum_{j \in J} a_{j} \log f_{j}=\sum_{j \in J} a_{j} \log \left(z_{j}+b_{j}^{1} t_{1}+b_{j}^{2} t_{2}\right) .
$$

The critical point equations are

$$
\frac{\partial \Phi}{\partial t_{1}}=\sum_{j \in J} a_{j} \frac{b_{j}^{1}}{f_{j}}=0, \quad \frac{\partial \Phi}{\partial t_{2}}=\sum_{j \in J} a_{j} \frac{b_{j}^{2}}{f_{j}}=0 .
$$

Introduce $H_{i}, i=1,2$, by the formula

$$
\frac{\partial \Phi}{\partial t_{i}}=\frac{H_{i}}{\prod_{j \in J} f_{j}}
$$

We have

$$
t_{1} \frac{\partial \Phi}{\partial t_{1}}+t_{2} \frac{\partial \Phi}{\partial t_{2}}=|a|-\sum_{i \in J} z_{i} \frac{a_{i}}{f_{i}}
$$

In other words, we have

$$
t_{1} H_{1}+t_{2} H_{2}=|a| \prod_{j \in J} f_{j}-\sum_{i \in J} z_{i} a_{i} \prod_{j \neq i} f_{j} .
$$

The critical set is

$$
C_{\Phi}(z)=\left\{t \in U(\mathcal{C}(z)) \mid \frac{\partial \Phi}{\partial t_{1}}=0, \frac{\partial \Phi}{\partial t_{2}}=0\right\}=\left\{t \in U(\mathcal{C}(z)) \mid H_{1}=0, H_{2}=0\right\}
$$

The algebra of functions on the critical set is

$$
A_{\Phi}(z)=\mathbb{C}(U(\mathcal{C}(z))) /\left\langle\frac{\partial \Phi}{\partial t_{1}}, \frac{\partial \Phi}{\partial t_{2}}\right\rangle=\mathbb{C}(U(\mathcal{C}(z))) /\left\langle H_{1}, H_{2}\right\rangle .
$$

Lemma 5.8. We have $\operatorname{dim} A_{\Phi}(z)=\left(\begin{array}{c}n-1 \\ 2\end{array}\right)$. 
Introduce elements $w_{i, j} \in A_{\Phi}(z)$ by the formula

$$
w_{i, j}=a_{i} a_{j}\left[\frac{d_{i, j}}{f_{i} f_{j}}\right] .
$$

Lemma 5.9. We have $w_{i, j}=-w_{j, i}$ and $\sum_{j \in J} w_{i, j}=0$.

Proof. The lemma follows from the identity

$$
a_{i} \frac{d f_{i}}{f_{i}} \wedge \frac{d \Phi}{\Phi}=\sum_{j \in J} a_{i} a_{j} \frac{d_{i, j}}{f_{i} f_{j}} d t_{1} \wedge d t_{2}+\sum_{m \in J} d z_{j} \wedge \mu_{j},
$$

where $\mu_{j}$ are suitable one-forms.

The elements $\left[\frac{a_{i}}{f_{i}}\right], i \in J$, generate $A_{\Phi}(z)$ by Lemma 2.4.

Lemma 5.10. For $j \in J$, we have the following identity in $A_{\Phi}(z)$,

$$
\sum_{i \in J} d_{i, j}\left[\frac{a_{i}}{f_{i}}\right]=0 .
$$

Lemma 5.11. We have

$$
\begin{gathered}
{\left[\frac{a_{i}}{f_{i}}\right] *_{z}\left[\frac{a_{j}}{f_{j}}\right]=\frac{1}{d_{i, j}} w_{i, j}, \quad i \neq j,} \\
{\left[\frac{a_{j}}{f_{j}}\right] *_{z}\left[\frac{a_{j}}{f_{j}}\right]=\sum_{i \notin\{j, k\}} \frac{d_{k, i}}{d_{j, k} d_{i, j}} w_{i, j}, \quad k \neq j .} \\
{\left[\frac{a_{i}}{f_{i}}\right] *_{z} w_{j, k}=\frac{d_{j, k}}{f_{i, j, k}}\left(a_{i} w_{j, k}+a_{j} w_{k, i}+a_{k} w_{i, j}\right), \quad \text { if } i \notin\{j, k\},} \\
{\left[\frac{a_{i}}{f_{i}}\right] *_{z} w_{j, i}=-\sum_{k \notin\{i, j\}}\left[\frac{a_{i}}{f_{i}}\right] *_{z} w_{j, k} .}
\end{gathered}
$$

Corollary 5.12. The elements $w_{i, j}, 1 \leqslant i<j \leqslant n$, span $A_{\Phi}(z)$.

Lemma 5.13. The identity element $[1](z) \in A_{\Phi}(z)$ satisfies the equations

$$
[1](z)=\frac{1}{|a|} \sum_{i \in J} z_{i}\left[\frac{a_{i}}{f_{i}}\right]=\frac{1}{|a|^{2}}\left(\sum_{i \in J} z_{i}\left[\frac{a_{i}}{f_{i}}\right]\right)^{2}=-\frac{1}{|a|^{2}} \sum_{1 \leqslant i<j \leqslant n} \frac{\left(z_{i} b_{j}^{1}-z_{j} b_{i}^{1}\right)^{2}}{d_{i, j} b_{i}^{1} b_{j}^{1}} w_{i, j} .
$$

Proof. To obtain the last expression in (5.39) we replace $\left[\frac{a_{i}}{f_{i}}\right]\left[\frac{a_{i}}{f_{i}}\right]$ with $\left[-\frac{a_{i}}{f_{i}} \sum_{j \neq i} \frac{b_{j}^{1}}{b_{i}^{1}} \frac{a_{j}}{f_{j}}\right]$.

Theorem 5.14. For any $k \in J$, the identity element $[1](z) \in A_{\Phi}(z)$ satisfies the equation

$$
[1](z)=\frac{1}{|a|^{2}} \sum^{\prime} \frac{f_{i, j, k}^{2}}{d_{i, j} d_{j, k} d_{k, i}} w_{i, j},
$$

where the sum is over all pairs $i<j$ such that $k \notin\{i, j\}$. 
Proof. The proof is the same as the proof of Lemma 5.6.

The canonical element is

$$
[E]=\sum_{1 \leqslant i<j \leqslant n}\left[\frac{d_{i, j}}{f_{i} f_{j}}\right] \otimes F_{i, j} \in A_{\Phi}(z) \otimes \operatorname{Sing} V .
$$

Theorem 5.15. The canonical isomorphism

$$
\alpha(z): A_{\Phi}(z) \rightarrow \operatorname{Sing} V
$$

is given by the formula

$$
w_{i, j} \mapsto v_{i, j}
$$

Corollary 5.16. We have $\alpha(z)[1])=q(z)$, where $q(z)$ is the conformal block of Theorem 5.7 .

5.6. Proof of Theorem [5.15. Introduce the coefficients $B_{i, j}$ by the formula

$$
\alpha(z)\left(w_{k, \ell}\right)=\sum_{1 \leqslant i<j \leqslant n} B_{i, j} F_{i, j} .
$$

We have

$$
-\frac{4 \pi^{2}}{a_{k} a_{\ell} d_{k, \ell} d_{i, j}} B_{i, j}=\sum_{p \in C_{\Phi}(z)} \operatorname{Res}_{p} \frac{1}{f_{k} f_{\ell} f_{i} f_{j}} \frac{\prod_{m \in J} f_{m}^{2}}{H_{1} H_{2}} .
$$

Lemma 5.17. We have $B_{i, j}=0$, if $\{i, j\} \cap\{k, \ell\}=\emptyset$.

Proof. The differential form

$$
\omega_{k, \ell, i, j}=\frac{\prod_{m \in J} f_{m}^{2}}{f_{k} f_{\ell} f_{i} f_{j} H_{1} H_{2}} d t_{1} \wedge d t_{2}
$$

has poles only on the curves $H_{1}=0$ and $H_{2}=0$. The poles are of first order. To calculate the right hand side in (5.45), we need to take the residue $\operatorname{Res}_{H_{1}=0} \omega_{k, \ell, i, j}$ of the form $\omega_{k, \ell, i, j}$ at the curve $H_{1}=0$ and then take the residue of that form on the curve $H_{1}=0$ at the points where $H_{2}=0$. This is the same as if we took with minus sign the residue of $\operatorname{Res}_{H_{1}=0} \omega_{k, \ell, i, j}$ on the curve $H_{1}=0$ at infinity. That residue at infinity with minus sign could be obtained differently in two steps. First we may take the residue $\operatorname{Res}_{\infty} \omega_{k, \ell, i, j}$ of $\omega_{k, \ell, i, j}$ at the line at infinity and then take the residue of that one-form on the line at infinity at the points where $H_{1}=0$.

So to calculate the right hand side in (5.45) we first calculate $\operatorname{Res}_{\infty} \omega_{k, \ell, i, j}$. The coordinates at infinity are $u_{1}=t_{1} / t_{2}, u_{2}=1 / t_{2}$. We have $f_{m}=\left(b_{m}^{1} u_{1}+b_{m}^{2}+u_{2} z_{m}\right) / u_{2}$. Denote $\tilde{f}_{m}\left(u_{1}\right)=$ $b_{m}^{1} u_{1}+b_{m}^{2}$. For $i=1,2$, we have $H_{i}\left(u_{1} / u_{2}, 1 / u_{2}\right)=\hat{H}_{i}\left(u_{1}, u_{2}\right) / u_{2}^{n-1}$, where $\hat{H}_{i}\left(u_{1}, u_{2}\right)$ are some polynomials. Denote $\tilde{H}_{i}\left(u_{1}\right)=\hat{H}_{i}\left(u_{1}, 0\right)$. We have $d t_{1} \wedge d t_{2}=-\frac{1}{u_{2}^{3}} d u_{1} \wedge d u_{2}$. Then the residue of $\omega_{k, \ell, i, j}$ at the line at infinity equals

$$
2 \pi \sqrt{-1} \tilde{\omega}_{k, \ell, i, j}=\frac{\prod_{m \in J} \tilde{f}_{m}(u)^{2}}{\tilde{f}_{k}(u) \tilde{f}_{\ell}(u) \tilde{f}_{i}(u) \tilde{f}_{j}(u) \tilde{H}_{1}(u) \tilde{H}_{2}(u)} d u,
$$

where $u=u_{1}$. On the line at infinity this one-form is holomorphic at $u=\infty$. The number $\frac{2 \pi \sqrt{-1}}{a_{k} a_{\ell} d_{k, \ell} d_{i, j}} B_{i, j}$ equals the sum of residues of the form $\tilde{\omega}_{k, \ell, i, j}$ at the points where $\tilde{H}_{1}(u)=0$. 
By formula (5.30), we have

$$
u \tilde{H}_{1}(u)+\tilde{H}_{2}(u)=|a| \prod_{m \in J} \tilde{f}_{m}(u) .
$$

Thus $\tilde{H}_{2}(u)=|a| \prod_{m \in J} \tilde{f}_{m}(u)$ at the point where $H_{1}(u)=0$. Therefore, the sum of residues of the form $\tilde{\omega}_{k, \ell, i, j}$ at the points where $\tilde{H}_{1}(u)=0$ equals the sum of residues of the form

$$
\tilde{\omega}_{k, \ell, i, j}=\frac{\prod_{m \notin\{k, \ell, i, j\}} \tilde{f}_{m}(u)}{|a| \tilde{H}_{1}(u)} d u
$$

at the points where $\tilde{H}_{1}(u)=0$. This sum is zero.

Lemma 5.18. We have $B_{k, j}=-\frac{a_{\ell}}{|a|}$, if $j \notin\{k, \ell\}$.

Proof. On the line at infinity we consider the differential one form

$$
\tilde{\omega}_{k, \ell, i, j}=\frac{\prod_{m \in J} \tilde{f}_{m}(u)^{2}}{\tilde{f}_{k}^{2}(u) \tilde{f}_{\ell}(u) \tilde{f}_{j}(u) \tilde{H}_{1}(u) \tilde{H}_{2}(u)} d u,
$$

As in Lemma 5.17 we observe that $\frac{2 \pi \sqrt{-1}}{a_{k} a_{\ell} d_{k, \ell} d_{k, j}} B_{k, j}$ equals the sum of residues of that one-form at the points where $H_{1}=0$. Consider the differential one-form

$$
\mu=\frac{\prod_{m \notin\{k, \ell, j\}} \tilde{f}_{m}(u)}{|a| \tilde{f}_{k}(u) \tilde{H}_{1}(u)} d u .
$$

As in Lemma 5.17 we observe that $\frac{2 \pi \sqrt{-1}}{a_{k} a_{\ell} d_{k, \ell} d_{k, j}} B_{k, j}$ equals the sum of residues of $\mu$ at the points where $\tilde{H}_{1}(u)=0$ and this sum equals

$$
-\operatorname{Res}_{\tilde{f}_{k}=0} \mu=-\frac{2 \pi \sqrt{-1}}{a_{k}|a| d_{k, \ell} d_{k, j}} .
$$

The lemma is proved.

By Lemmas 5.17 and 5.18 we know that $\alpha(z)\left(w_{k, \ell}\right)=B_{k, \ell} F_{k, \ell}-\frac{a_{k}}{|a|} \sum_{i \neq k} F_{i, \ell}-\frac{a_{\ell}}{|a|} \sum_{j \neq \ell} F_{k, j}$. From the condition that $\alpha(z)\left(w_{k, \ell}\right) \in \operatorname{Sing} V$ we conclude that $B_{k, \ell}=\frac{|a|-a_{k}-a_{\ell}}{|a|}$. The theorem is proved.

5.7. Contravariant map as the inverse to the canonical map. The canonical map $\alpha(z): A_{\Phi}(z) \rightarrow \operatorname{Sing} V$ is the isomorphism described in Theorem 5.15, The contravariant map $\mathcal{S}^{(a)}: V \rightarrow V^{*}$ is defined by the formula $F_{i, j} \mapsto a_{i} a_{j}\left(H_{i}, H_{j}\right)$. By identifying $a_{i} a_{j}\left(H_{i}, H_{j}\right)$ with the differential form $a_{i} a_{j} \frac{d_{i, j}}{f_{i} f_{j}} d t_{1} \wedge d t_{2}$ and then projecting the coefficient to $A_{\Phi}(z)$ we obtain the map

$$
\left[\mathcal{S}^{(a)}\right]: V \rightarrow A_{\Phi}(z), \quad F_{i, j} \mapsto w_{i, j}=a_{i} a_{j}\left[\frac{d_{i, j}}{f_{i} f_{j}}\right]
$$

Theorem 5.19. The composition $\alpha(z) \circ\left[\mathcal{S}^{(a)}\right]: V \rightarrow \operatorname{Sing} V$ is the orthogonal projection. The composition $\left[\mathcal{S}^{(a)}\right] \circ \alpha(z): A_{\Phi}(z) \rightarrow A_{\Phi}(z)$ is the identity map. 
Proof. The composition $\alpha(z) \circ\left[\mathcal{S}^{(a)}\right]$ sends $F_{i, j}$ to $v_{i, j}$ which is the orthogonal projection by Lemma 5.3. The composition $\left[\mathcal{S}^{(a)}\right] \circ \alpha(z)$ sends $w_{i, j}$ to

$$
w_{i, j}-\frac{a_{i}}{|a|} \sum_{k \in J} w_{k, j}-\frac{a_{j}}{|a|} \sum_{\ell \in J} w_{i, \ell}
$$

The last two sums are equal to zero in $A_{\Phi}(z)$ by Lemma 5.9.

\subsection{Corollaries of Theorem $\mathbf{5 . 1 5}$.}

Theorem 5.20. For any $j \in J$, the Sing $V$-valued function $\frac{\partial q}{\partial z_{j}}(z)$ satisfies the Gauss-Manin differential equations with $\kappa=|a|$,

$$
|a| \frac{\partial}{\partial z_{i}} \frac{\partial q}{\partial z_{j}}(z)=K_{i}(z) \frac{\partial q}{\partial z_{j}}(z), \quad i \in J
$$

Proof. By Theorems 5.14 and 5.15, we have

$$
q(z)=\alpha(z)([1](z))=\frac{1}{|a|^{2}} \alpha(z)\left(\left(\sum_{m \in J} z_{m}\left[\frac{a_{m}}{f_{m}}\right]\right)^{2}\right)=\frac{1}{|a|^{2}} \sum_{m, \ell \in J} z_{m} z_{\ell} \alpha(z)\left(\left[\frac{a_{m}}{f_{m}}\right]\left[\frac{a_{\ell}}{f_{\ell}}\right]\right) .
$$

By Theorem 5.15, for any $m, \ell \in J$, the element $\alpha(z)\left(\left[\frac{a_{m}}{f_{m}}\right]\left[\frac{a_{\ell}}{f_{\ell}}\right]\right) \in \operatorname{Sing} V$ is a linear combination of vectors $v_{i, j}$ with constant coefficients. Hence

$$
\begin{gathered}
\frac{\partial^{2} q}{\partial z_{j} \partial z_{i}}(z)=\frac{2}{|a|^{2}} \alpha(z)\left(\left[\frac{a_{i}}{f_{i}}\right]\left[\frac{a_{j}}{f_{j}}\right]\right), \\
\frac{\partial q}{\partial z_{j}}(z)=\frac{2}{|a|} \alpha(z)\left(\left\lceil\frac{a_{j}}{f_{j}}\right] *_{z} \frac{1}{|a|} \sum_{m \in J} z_{m}\left[\frac{a_{m}}{f_{m}}\right]\right)=\frac{2}{|a|} \alpha(z)\left(\left[\frac{a_{j}}{f_{j}}\right]\right), \\
K_{i}(z) \frac{\partial q}{\partial z_{j}}(z)=\frac{2}{|a|} \alpha(z)\left(\left[\frac{a_{i}}{f_{i}}\right]\left[\frac{a_{j}}{f_{j}}\right]\right) .
\end{gathered}
$$

This implies the theorem.

Corollary 5.21. Conjectures 3.7 and 3.8 hold for this family of arrangements.

The tangent morphism $\beta$ and the residue form on the bundle of algebras induce a holomorphic bilinear form $\eta$ on fibers of the tangent bundle,

$$
\begin{aligned}
\eta\left(\partial_{i}, \partial_{j}\right)_{z} & =\left(\beta(z)\left(\partial_{i}\right), \beta(z)\left(\partial_{j}\right)\right)_{z}=(-1)^{k} S^{(a)}\left(\alpha(z) \beta(z)\left(\partial_{i}\right), \alpha(z) \beta(z)\left(\partial_{j}\right)\right)= \\
& =\left(\left[\frac{a_{i}}{f_{i}}\right],\left[\frac{a_{j}}{f_{j}}\right]\right)_{z}=(-1)^{k} S^{(a)}\left(\alpha(z)\left(\left[\frac{a_{i}}{f_{i}}\right]\right), \alpha(z)\left(\left[\frac{a_{j}}{f_{j}}\right]\right)\right) .
\end{aligned}
$$

By Theorem 3.10, we have

$$
\eta\left(\partial_{i}, \partial_{j}\right)_{z}=\frac{|a|^{2}}{4} S^{(a)}\left(\frac{\partial q}{\partial z_{i}}(z), \frac{\partial q}{\partial z_{j}}(z)\right) .
$$

Theorems 3.12 and 3.13 also hold for this family of arrangements. 
Theorem 5.22. Recall the potential function of first kind $P(z)=S^{(a)}(q(z), q(z))$. We have

$$
P(z)=\sum_{1 \leqslant i<j<k \leqslant n} \frac{a_{i} a_{j} a_{k}}{|a|^{5}} \frac{f_{i, j, k}^{4}}{d_{i, j}^{2} d_{j, k}^{2} d_{k, i}^{2}} .
$$

Proof. By Theorem 3.11, for any $r \leqslant 4$, we have

$$
\left(\beta(z)\left(\partial_{m_{1}}\right) *_{z} \cdots *_{z} \beta(z)\left(\partial_{m_{r}}\right),[1](z)\right)_{z}=\frac{|a|^{r}}{A_{2, r}} \frac{\partial^{r} P}{\partial z_{m_{1}} \ldots \partial z_{m_{r}}}(z),
$$

for all $m_{1}, \ldots, m_{r} \in J$. In particular,

$$
\left(\beta(z)\left(\partial_{k}\right) *_{z} \beta(z)\left(\partial_{\ell}\right) *_{z} \beta(z)\left(\partial z_{m}\right),[1](z)\right)_{z}=\frac{|a|^{3}}{4 !} \frac{\partial^{3} P}{\partial z_{k} \partial z_{\ell} \partial z_{m}}(z)
$$

for all $k, \ell, m \in J$. Introduce the function

$$
\hat{P}(z)=\frac{1}{4 !} \sum_{1 \leqslant i<j<k \leqslant n} \frac{a_{i} a_{j} a_{k}}{|a|^{2}} \frac{f_{i, j, k}^{4}}{d_{i, j}^{2} d_{j, k}^{2} d_{k, i}^{2}} .
$$

Proposition 5.23. We have

$$
\left(\beta(z)\left(\partial_{j}\right) *_{z} \beta(z)\left(\partial_{\ell}\right) *_{z} \beta(z)\left(\partial z_{m}\right),[1](z)\right)_{z}=\frac{\partial^{3} \hat{P}}{\partial z_{j} \partial z_{\ell} \partial z_{m}}(z)
$$

for all $k, \ell, m \in J$.

Proof. For $k, \ell \in J$, we define the differential one-form $\psi_{k, \ell}$ on $\mathbb{C}^{n}-\Delta$ by the formula

$$
\psi_{k, \ell}\left(\partial_{m}\right)=\left(\beta(z)\left(\partial_{k}\right) *_{z} \beta(z)\left(\partial_{\ell}\right), \beta(z)\left(\partial_{m}\right)\right)_{z}
$$

The canonical isomorphism identifies the residue form and the contravariant form and therefore we may write

$$
\psi_{k, \ell}\left(\partial_{m}\right)=S^{(a)}\left(\alpha(z) \beta(z)\left(\partial_{k}\right) *_{z} \alpha(z) \beta(z)\left(\partial_{\ell}\right), \alpha(z) \beta(z)\left(\partial_{m}\right)\right) .
$$

Lemma 5.24. The form $\psi_{k, \ell}$ is the differential of the function

$$
\varphi_{k, \ell}(z)=\frac{|a|}{2} \frac{1}{d_{k, \ell}} S^{(a)}\left(v_{k, \ell}, q(z)\right)
$$

if $k \neq \ell$, and of the function

$$
\varphi_{k, \ell}(z)=\frac{|a|}{2} \sum_{i \notin\{j, k\}} \frac{d_{j, i}}{d_{k, j} d_{i, k}} S^{(a)}\left(v_{i, k}, v\right),
$$

if $k=\ell$, where $j$ is any number in $J$ such that $j \neq k$.

Proof. The vector $\alpha(z) \beta(z)\left(\partial_{k}\right) *_{z} \alpha(z) \beta(z)\left(\partial_{\ell}\right)=\alpha(z)\left(\left[\frac{a_{k}}{f_{k}}\right]\left[\frac{a_{\ell}}{f_{\ell}}\right]\right) \in \operatorname{Sing} V$ equals $\frac{1}{d_{k, \ell}} v_{k, \ell}$ if $k \neq \ell$ and equals $\sum_{i \notin\{j, k\}} \frac{d_{j, i}}{d_{k, j} d_{i, k}} v_{i, k}$ if $k=\ell$. We also have $\alpha(z) \beta(z)\left(\partial_{m}\right)=\frac{|a|}{2} \frac{\partial q}{\partial z_{m}}$. This implies the lemma. 
Proposition 5.23 is equivalent to the formula

$$
\frac{\partial^{2} \hat{P}}{\partial z_{k} \partial z_{\ell}}=\varphi_{k, \ell}
$$

for all $k, \ell \in J$. The proof of (5.67) is by direct verification. Namely, assume that $k<\ell$. Then

$$
\frac{\partial^{2} \hat{P}}{\partial z_{k} \partial z_{\ell}}=\frac{a_{i} a_{k} a_{\ell}}{2|a|^{2}}\left(\sum_{i<k} \frac{f_{i, k, l}^{2}}{d_{i, k} d_{k, \ell} d_{\ell, i}} \frac{1}{d_{k, \ell}}+\sum_{k<i<\ell} \frac{f_{k, i, l}^{2}}{d_{k, i} d_{i, \ell} d_{\ell, k}} \frac{1}{d_{\ell, k}}+\sum_{i>\ell} \frac{f_{k, \ell, i}^{2}}{d_{k, \ell} d_{\ell, i} d_{i, k}} \frac{1}{d_{k, \ell}}\right) .
$$

We also have

$$
\begin{aligned}
\varphi_{k, \ell} & =\frac{1}{2|a|} S^{(a)}\left(\frac{1}{d_{k, \ell}} v_{k, \ell}, \sum_{\substack{i<j \\
k \notin\{i, j\}}} \frac{f_{i, j, k}^{2}}{d_{i, j} d_{j, k} d_{k, i}} v_{i, j}\right)= \\
& =\frac{1}{2|a| d_{k, \ell}} S^{(a)}\left(v_{k, \ell}, \sum_{i<k} \frac{f_{i, \ell, k}^{2}}{d_{i, \ell} d_{\ell, k} d_{k, i}} v_{i, \ell}+\sum_{k<i<\ell} \frac{f_{i, \ell, k}^{2}}{d_{i, \ell} d_{\ell, k} d_{k, i}} v_{i, \ell}+\sum_{i>\ell} \frac{f_{\ell, i, k}^{2}}{d_{\ell, i} d_{i, k} d_{k, \ell}} v_{\ell, i}\right) \\
& =\frac{a_{i} a_{\ell} a_{k}}{2|a|^{2} d_{k, \ell}}\left(-\sum_{i<k} \frac{f_{i, \ell, k}^{2}}{d_{i, \ell} d_{\ell, k} d_{k, i}}-\sum_{k<i<\ell} \frac{f_{i, \ell, k}^{2}}{d_{i, \ell} d_{\ell, k} d_{k, i}}+\sum_{i>\ell} \frac{f_{\ell, i, k}^{2}}{d_{\ell, i} d_{i, k} d_{k, \ell}}\right) .
\end{aligned}
$$

Comparing (5.68) and (5.68) we conclude that (5.67) holds if $k<\ell$. Assume that $k=\ell$. Then

$$
\frac{\partial^{2} \hat{P}}{\partial z_{k}^{2}}=\sum_{\substack{i<j \\ k \notin\{i, j\}}} \frac{a_{i} a_{j} a_{k}}{2|a|^{2}} \frac{f_{i, j, k}^{2}}{d_{j, k}^{2} d_{k, i}^{2}}
$$

We also have

$$
\begin{aligned}
\varphi_{k, \ell} & =\frac{1}{2|a|} S^{(a)}\left(\sum_{m \notin\{k, \ell\}} \frac{d_{\ell, m}}{d_{k, \ell} d_{m, k}} v_{m, k}, \sum_{\substack{i<j \\
k \notin\{i, j\}}} \frac{f_{i, j, k}^{2}}{d_{i, j} d_{j, k} d_{k, i}} v_{i, j}\right)= \\
& =\sum_{\substack{i \leq j \\
k \notin\{i, j\}}} \frac{1}{2|a|} \frac{f_{i, j, k}^{2}}{d_{i, j} d_{j, k} d_{k, i}} S^{(a)}\left(\frac{d_{\ell, i}}{d_{k, \ell} d_{i, k}} v_{i, k}+\frac{d_{\ell, j}}{d_{k, \ell} d_{j, k}} v_{j, k}, v_{i, j}\right)= \\
& =\sum_{\substack{i<j \\
k \notin\{i, j\}}} \frac{a_{i} a_{j} a_{k}}{2|a|^{2} d_{k, \ell}} \frac{f_{i, j, k}^{2}}{d_{i, j} d_{j, k} d_{k, i}}\left(-\frac{d_{\ell, i}}{d_{i, k}}+\frac{d_{\ell, j}}{d_{j, k}}\right)= \\
& =\sum_{\substack{i<j \\
k \notin\{i, j\}}} \frac{a_{i} a_{j} a_{k}}{2|a|^{2} d_{k, \ell}} \frac{f_{i, j, k}^{2}}{d_{i, j} d_{j, k} d_{k, i}} \frac{d_{\ell, k} d_{i, j}}{d_{i, k} d_{j, k}}=\sum_{\substack{i<j \\
k \notin\{i, j\}}} \frac{a_{i} a_{j} a_{k}}{2|a|^{2}} \frac{f_{i, j, k}^{2}}{d_{j, k}^{2} d_{k, i}^{2}} .
\end{aligned}
$$

Comparing (5.70) and (5.71) we conclude that (5.67) holds for $k=\ell$. The proposition is proved. 
Both functions $|a|^{3} P(z) / 4$ ! and $\hat{P}(z)$ satisfy the same equation and both functions are homogeneous polynomials in $z$ of degree four. Hence $|a|^{3} P(z) / 4 !=\hat{P}(z)$. Thus the proposition implies the theorem.

The period map $q: \mathbb{C}^{n}-\Delta \rightarrow \mathbb{C}^{n}-\Delta$ is a polynomial map in $z$ of degree two with respect to the combinatorial connection.

The space Sing $V$ has distinguished bases labeled by $k \in J$. The basis corresponding to $k$ consists of the vectors $v_{i, j}$ such that $1 \leqslant i<j \leqslant n$ and $k \notin\{i, j\}$. Such a basis defines coordinate hyperplanes in $\operatorname{Sing} V$.

Lemma 5.25. The period map sends the discriminant $\Delta \subset \mathbb{C}^{n}$ to the union $\Delta_{V} \subset \operatorname{Sing} V$ of all coordinate hyperplanes of all distinguished bases in $\operatorname{Sing} V$.

Proof. The period map is given by the formula $q(z)=\frac{1}{|a|^{2}} \sum^{\prime} \frac{f_{i, j, k}^{2}}{d_{i, j} d_{j, k} d_{k, i}} v_{i, j}$, where the sum is over all pairs $i<j$ such that $k \notin\{i, j\}$. Thus the functions $\frac{f_{i, j, k}^{2}}{d_{i, j} d_{j, k} d_{k, i}}$ are the coordinate functions of the period map in this (combinatorially flat) basis. The lemma follows from this description of the coordinate functions.

Lemma 5.26. For $z \in \mathbb{C}^{n}-\Delta$, the kernel of the differential of the period map is two dimensional. The kernel is spanned by the vectors

$$
\sum_{j \neq i} d_{j, i} \partial_{j}, \quad i \in J
$$

Any two of these vectors are linearly independent.

Introduce the potential function of second kind

$$
\tilde{P}\left(z_{1}, \ldots, z_{n}\right)=\frac{1}{4 !} \sum_{1 \leqslant i<j<k \leqslant n} \frac{a_{i} a_{j} a_{k}}{d_{i, j}^{2} d_{j, k}^{2} d_{k, i}^{2}} f_{i, j, k}^{4} \log f_{i, j, k} .
$$

Theorem 5.27. For any $m_{0}, \ldots, m_{4} \in J$ we have

$$
\frac{\partial^{5} \tilde{P}}{\partial z_{m_{0}} \cdots \partial z_{m_{4}}}(z)=\left(\left[\frac{a_{m_{0}}}{f_{m_{0}}}\right] *_{z} \cdots *_{z}\left[\frac{a_{m_{4}}}{f_{m_{4}}}\right],[1](z)\right)_{z} .
$$

Theorem 5.27 proves Conjecture 3.14 for this family of arrangements.

If $m_{1} \neq m_{2}$ and $m_{3} \neq m_{4}$, equation (5.74) takes the form

$$
S^{(a)}\left(K_{m_{0}}(z) v_{m_{1}, m_{2}}, v_{m_{3}, m_{4}}\right)=d_{m_{1}, m_{2}} d_{m_{3}, m_{4}} \frac{\partial^{5} \tilde{P}}{\partial z_{m_{0}} \ldots \partial z_{m_{4}}}(z) .
$$

Corollary 5.28. The matrix elements of the operators $K_{i}(z)$ with respect to the (combinatorially constant) vectors $v_{i, j}$ are described by the fifth derivatives of the potential function of second kind.

Notice that

$$
S^{(a)}\left(v_{m_{1}, m_{2}}, v_{m_{3}, m_{4}}\right)=d_{m_{1}, m_{2}} d_{m_{3}, m_{4}} \frac{|a|^{4}}{4 !} \frac{\partial^{4} P}{\partial z_{m_{1}} \ldots \partial z_{m_{4}}}(z),
$$

where $P(z)$ is the potential function of first kind, see Theorem 3.11. 
Proof of Theorem 5.27. We have the relation $\sum_{j \in J} d_{i, j}\left[\frac{a_{j}}{f_{j}}\right]=0$ for any $i \in J$, see (5.36), and the relation

$$
\sum_{j \in J} d_{i, j} \frac{\partial}{\partial z_{j}} \frac{\partial^{4} P}{\partial z_{m_{1}} \ldots \partial z_{m_{4}}}(z)=0
$$

for any $m_{1}, \ldots, m_{4}, i \in J$. By using these two relations and by reordering the set $J$ if necessary, we can reduce formula (5.74) to three cases in which $\left(m_{0}, \ldots, m_{4}\right)$ equals $(5,1,2,3,4)$ or $(3,1,2,3,4)$ or $(3,1,2,1,2)$.

Let $\left(m_{0}, \ldots, m_{4}\right)=(5,1,2,3,4)$. Then $\frac{\partial^{5} \tilde{P}}{\partial z_{m_{0}} \ldots \partial z_{m_{4}}}(z)=0$ and

$$
\begin{gathered}
\left(\left[\frac{a_{m_{0}}}{f_{m_{0}}}\right] *_{z} \cdots *_{z}\left[\frac{a_{m_{4}}}{f_{m_{4}}}\right],[1](z)\right)_{z}=\frac{1}{d_{1,2} d_{3,4}} S^{(a)}\left(K_{5}(z) v_{1,2}, v_{3,4}\right)= \\
\quad=\frac{d_{1,2}}{d_{1,2} d_{3,4} f_{5,1,2}} S^{(a)}\left(a_{5} v_{1,2}+a_{1} v_{2,5}+a_{2} v_{5,1}, v_{3,4}\right)=0 .
\end{gathered}
$$

Let $\left(m_{0}, \ldots, m_{4}\right)=(3,1,2,3,4)$. Then $\frac{\partial^{5} \tilde{P}}{\partial z_{m_{0}} \ldots \partial z_{m_{4}}}(z)=0$ and

$$
\begin{aligned}
& \left(\left[\frac{a_{m_{0}}}{f_{m_{0}}}\right] *_{z} \cdots *_{z}\left[\frac{a_{m_{4}}}{f_{m_{4}}}\right],[1](z)\right)_{z}=\frac{1}{d_{1,2} d_{3,4}} S^{(a)}\left(K_{3}(z) v_{1,2}, v_{3,4}\right)= \\
& \quad=\frac{d_{1,2}}{d_{1,2} d_{3,4} f_{3,1,2}} S^{(a)}\left(a_{3} v_{1,2}+a_{1} v_{2,3}+a_{2} v_{3,1}, v_{3,4}\right)= \\
& \quad=\frac{d_{1,2}}{d_{1,2} d_{3,4} f_{3,1,2}}\left(0+a_{1} \frac{a_{2} a_{3} a_{4}}{|a|}-a_{2} \frac{a_{1} a_{3} a_{4}}{|a|}\right)=0 .
\end{aligned}
$$

Let $\left(m_{0}, \ldots, m_{4}\right)=(3,1,2,1,2)$. Then $\frac{\partial^{5} \tilde{P}}{\partial z_{m_{0}} \ldots \partial z_{m_{4}}}(z)=\frac{a_{1} a_{2} a_{3}}{d_{1,2} f_{1,2,3}}$ and

$$
\begin{aligned}
& \left(\left[\frac{a_{m_{0}}}{f_{m_{0}}}\right] *_{z} \cdots *_{z}\left[\frac{a_{m_{4}}}{f_{m_{4}}}\right],[1](z)\right)_{z}=\frac{1}{d_{1,2} d_{1,2}} S^{(a)}\left(K_{3}(z) v_{1,2}, v_{1,2}\right)= \\
& \quad=\frac{d_{1,2}}{d_{1,2} d_{1,2} f_{3,1,2}} S^{(a)}\left(a_{3} v_{1,2}+a_{1} v_{2,3}+a_{2} v_{3,1}, v_{1,2}\right)= \\
& \quad=\frac{d_{1,2}}{d_{1,2} d_{1,2} f_{3,1,2}}\left(a_{3} \frac{a_{1} a_{2} \sum_{j \notin\{1,2\}} a_{j}}{|a|}+a_{1} \frac{a_{1} a_{2} a_{3}}{|a|}+a_{2} \frac{a_{1} a_{2} a_{3}}{|a|}\right)=\frac{a_{1} a_{2} a_{3}}{d_{1,2} f_{1,2,3}} .
\end{aligned}
$$

The theorem is proved.

5.9. Frobenius like structure. Consider the quotient $M$ of $\mathbb{C}^{n}$ by the two-dimensional subspace, which is the kernel of the period map, see Lemma 5.26. Let $\pi: \mathbb{C}^{n} \rightarrow M$ be the natural projection. Then all our objects descend to the quotient and form on $M-\pi(\Delta)$ a structure which we will also call a Forbenius like structure.

\section{Generic arrangements in $\mathbb{C}^{k}$}

6.1. An arrangement in $\mathbb{C}^{n} \times \mathbb{C}^{k}$. Consider $\mathbb{C}^{k}$ with coordinates $t_{1}, \ldots, t_{k}$, $\mathbb{C}^{n}$ with coordinates $z_{1}, \ldots, z_{n}$. Fix $n$ linear functions on $\mathbb{C}^{k}, g_{j}=\sum_{m=1}^{k} b_{j}^{m} t_{m}, j \in J, b_{j}^{m} \in \mathbb{C}$. For $i_{1}, \ldots, i_{k} \subset J$, denote

$$
d_{i_{1}, \ldots, i_{k}}=\operatorname{det}_{\ell, m=1}^{k}\left(b_{i_{\ell}}^{m}\right)
$$


We assume that all the numbers $d_{i_{1}, \ldots, i_{k}}$ are nonzero if $i_{1}, \ldots, i_{k}$ are distinct. In other words we assume that the collection of functions $g_{j}, j \in J$, is generic. We define $n$ linear functions on $\mathbb{C}^{n} \times \mathbb{C}^{k}, f_{j}=z_{j}+g_{j}, j \in J$. In $\mathbb{C}^{n} \times \mathbb{C}^{k}$ we define the arrangement $\tilde{\mathcal{C}}=\left\{\tilde{H}_{j} \mid f_{j}=0, j \in J\right\}$.

For every $z=\left(z_{1}, \ldots, z_{n}\right)$ the arrangement $\tilde{\mathcal{C}}$ induces an arrangement $\mathcal{C}(z)$ in the fiber of the projection $\mathbb{C}^{n} \times \mathbb{C}^{k} \rightarrow \mathbb{C}^{n}$ over $z$. We identify every fiber with $\mathbb{C}^{k}$. Then $\mathcal{C}(z)$ consists of hyperplanes $H_{j}(z), j \in J$, defined in $\mathbb{C}^{k}$ by the equations $f_{j}=0$. Denote $U(\mathcal{C}(z))=$ $\mathbb{C}^{k}-\cup_{j \in J} H_{j}(z)$, the complement to the arrangement $\mathcal{C}(z)$.

The arrangement $\mathcal{C}(z)$ is with normal crossings if and only if $z \in \mathbb{C}^{n}-\Delta$,

$$
\Delta=\cup_{\left\{i_{1}<\cdots<i_{k+1}\right\} \subset J} H_{i_{1}, \ldots, i_{k+1}},
$$

where $H_{i_{1}, \ldots, i_{k+1}}$ is the hyperplane defined by the equation $f_{i_{1}, \ldots, i_{k+1}}=0$,

$$
f_{i_{1}, \ldots, i_{k+1}}=\sum_{m=1}^{k+1}(-1)^{m-1} z_{i_{m}} d_{i_{1}, \ldots, \widehat{i_{m}}, \ldots, i_{k+1}} .
$$

6.2. Good fibers. For any $z \in \mathbb{C}^{n}-\Delta$, the space $\mathcal{A}^{k}(\mathcal{C}(z))$ has the standard basis $\left(H_{i_{1}}(z), \ldots, H_{i_{k}}(z)\right), 1 \leqslant i_{1}<\cdots<i_{k} \leqslant n$. The space $\mathcal{F}^{k}(\mathcal{C}(z))$ has the standard dual basis $F\left(H_{i_{1}}(z), \ldots, H_{i_{k}}(z)\right)$. For $z^{1}, z^{2} \in \mathbb{C}^{n}-\Delta$, the combinatorial connection identifies the spaces $\mathcal{A}^{k}\left(\mathcal{C}\left(z^{1}\right)\right), \mathcal{F}^{k}\left(\mathcal{C}\left(z^{1}\right)\right)$ with the spaces $\mathcal{A}^{k}\left(\mathcal{C}\left(z^{2}\right)\right), \mathcal{F}^{k}\left(\mathcal{C}\left(z^{2}\right)\right)$, respectively, by identifying the corresponding standard bases.

Assume that nonzero weights $\left(a_{j}\right)_{j \in J}$ are given. Then each arrangement $\mathcal{C}(z)$ is weighted. For $z \in \mathbb{C}^{n}-\Delta$, the arrangement $\mathcal{C}(z)$ is unbalanced if $|a| \neq 0$. We assume that $|a| \neq 0$.

For $z \in \mathbb{C}^{n}-\Delta$, we denote $V=\mathcal{F}^{k}(\mathcal{C}(z)), V^{*}=\left(\mathcal{F}^{k}(\mathcal{C}(z))^{*}=\mathcal{A}^{k}(\mathcal{C}(z)), F_{i_{1}, \ldots, i_{k}}=\right.$ $F\left(H_{i_{1}}(z), \ldots, H_{i_{k}}(z)\right)$. For any permutation $\sigma \in \Sigma_{k}$, we have $F_{i_{\sigma(1)}, \ldots, i_{\sigma(k)}}=(-1)^{\sigma} F_{i_{1}, \ldots, i_{k}}$. If $v=\sum_{1 \leqslant i_{1}<\cdots<i_{k} \leqslant n} c_{i_{1}, \ldots, i_{k}} F_{i_{1}, \ldots, i_{k}}$ is a vector of $V$, we introduce $c_{i_{1}, \ldots, i_{k}}$ for all $i_{1}, \ldots, i_{k} \in J$ by the rule: $c_{i_{\sigma(1)}, \ldots, i_{\sigma(k)}}=(-1)^{\sigma} c_{i_{1}, \ldots, i_{k}}$. The contravariant form on $V$ is defined by

$$
\begin{aligned}
& S^{(a)}\left(F_{i_{1}, \ldots, i_{k}}, F_{j_{1}, \ldots, j_{k}}\right)=0, \quad \text { if }\left\{i_{1}, \ldots, i_{k}\right\} \neq\left\{i_{1}, \ldots, i_{k}\right\}, \\
& S^{(a)}\left(F_{i_{1}, \ldots, i_{k}}, F_{i_{1}, \ldots, i_{k}}\right)=\prod_{m=1}^{k} a_{i_{m}}
\end{aligned}
$$

the singular subspace is defined by

$$
\text { Sing } V=\left\{\sum_{1 \leqslant i_{1}<\cdots<i_{k} \leqslant n} c_{i_{1}, \ldots, i_{k}} F_{i_{1}, \ldots, i_{k}} \mid \sum_{j \in J} a_{j} c_{j, j_{1}, \ldots, j_{k-1}}=0 \text { for all }\left\{j_{1}, \ldots, j_{k-1}\right\} \subset J\right\} .
$$

By Corollary 2.8, the restriction of $S^{(a)}$ to $\operatorname{Sing} V$ is nondegenerate. Denote $(\operatorname{Sing} V)^{\perp}$ the orthogonal complement to $\operatorname{Sing} V$ with respect to $S^{(a)}$. Then $V=\operatorname{Sing} V \oplus(\operatorname{Sing} V)^{\perp}$. Denote $\pi: V \rightarrow \operatorname{Sing} V$ the orthogonal projection.

Lemma 6.1. The space $(\operatorname{Sing} V)^{\perp}$ is generated by vectors

$$
\sum_{j \in J} F_{j, j_{1}, \ldots, j_{k-1}}
$$

labeled by subsets $\left\{j_{1}, \ldots, j_{k-1}\right\} \subset J$. 
For distinct $i_{1}, \ldots, i_{k}$, we define the vector $v_{i_{1}, \ldots, i_{k}} \in V$ by the formula

$$
v_{i_{1}, \ldots, i_{k}}=F_{i_{1}, \ldots, i_{k}}-\sum_{m=1}^{k} \frac{a_{i_{m}}}{|a|} \sum_{j \in J} F_{i_{1}, \ldots, i_{m-1}, j, i_{m+1}, \ldots, i_{k}} .
$$

We have $v_{i_{\sigma(1)}, \ldots, i_{\sigma(k)}}=(-1)^{\sigma} v_{i_{1}, \ldots, i_{k}}$. Set $v_{i_{1}, \ldots, i_{k}}=0$ if $i_{1}, \ldots, i_{k}$ are not distinct.

Lemma 6.2. We have the following properties.

(i) $\operatorname{dim} \operatorname{Sing} V=\left(\begin{array}{c}n-1 \\ k\end{array}\right)$.

(ii) For distinct $i_{1}, \ldots, i_{k}$, we have $v_{i_{1}, \ldots, i_{k}} \in \operatorname{Sing} V$ and $v_{i_{1}, \ldots, i_{k}}=\pi\left(F_{i_{1}, \ldots, i_{k}}\right)$.

(iii) For $\left\{j_{1}, \ldots, j_{k-1}\right\} \subset J$, we have $\sum_{j \in J} v_{j_{1}, \ldots, j_{k-1}, j}=0$.

(iv) For any $m \in J$, the set $v_{i_{1}, \ldots, i_{k}}, 1 \leqslant i_{1}<\cdots<i_{k} \leqslant n, m \notin\left\{i_{1}, \ldots, i_{k}\right\}$, is a basis of Sing $V$.

Lemma 6.3. We have

$$
\begin{aligned}
& S^{(a)}\left(v_{i_{1}, \ldots, i_{k}}, v_{j_{1}, \ldots, j_{k}}\right)=0, \quad \text { if }\left|\left\{i_{1}, \ldots, i_{k}\right\} \cap\left\{j_{1}, \ldots, j_{k}\right\}\right|<k-1, \\
& S^{(a)}\left(v_{i_{1}, \ldots, i_{k-1}, i_{k}}, v_{i_{1}, \ldots, i_{k-1}, i_{k+1}}\right)=-\frac{\prod_{\ell=1}^{k+1} a_{i_{\ell}}}{|a|} \quad \text { for distinct } i_{1}, \ldots, i_{k-1}, i_{k}, i_{k+1}, \\
& S^{(a)}\left(v_{i_{1}, \ldots, i_{k}}, v_{i_{1}, \ldots, i_{k}}\right)=\frac{\left(\sum_{\ell \notin\left\{i_{1}, \ldots, i_{k}\right\}} a_{i_{\ell}}\right) \prod_{m=1}^{k} a_{i_{m}}}{|a|} .
\end{aligned}
$$

Proof. The lemma is a straightforward corollary of (6.4) and (6.7).

6.3. Operators $K_{i}(z): V \rightarrow V$. For any subset $\left\{i_{1}, \ldots, i_{k+1}\right\} \subset J$, we define the linear operator $L_{i_{1}, \ldots, i_{k+1}}: V \rightarrow V$ by the formula

$$
\begin{aligned}
F_{i_{1}, \ldots, \widehat{i_{m}}, \ldots, i_{k+1}} & \mapsto(-1)^{m} \sum_{\ell=1}^{k+1}(-1)^{\ell} a_{i_{\ell}} F_{i_{1}, \ldots, \widehat{i_{\ell}}, \ldots, i_{k+1}}, \quad m=1, \ldots, k+1, \\
F_{j_{1}, \ldots, j_{k}} & \mapsto 0, \quad \text { if }\left\{j_{1}, \ldots, j_{k}\right\} \text { is not a subset of }\left\{i_{1}, \ldots, i_{k+1}\right\},
\end{aligned}
$$

see formula (3.5). Notice that $L_{i_{1}, \ldots, i_{k+1}}$ does not depend on the order of $i_{1}, \ldots, i_{k+1}$.

We define the operators $K_{i}(z): V \rightarrow V, i \in J$, by the formula

$$
K_{i}(z)=\sum \frac{d_{i_{1}, \ldots, i_{k}}}{f_{i, i_{1}, \ldots, i_{k}}} L_{i, i_{1}, \ldots, i_{k}}
$$

where the sum is over all unordered subsets $\left\{i_{1}, \ldots, i_{k}\right\} \subset J-\{i\}$, see formula (3.6). For any $i \in J$ and $z \in \mathbb{C}^{n}-\Delta$, the operator $K_{i}(z)$ preserves the subspace $\operatorname{Sing} V \subset V$ and is a symmetric operator, $S^{(a)}\left(K_{i}(z) v, w\right)=S^{(a)}\left(v, K_{i}(z) w\right)$ for all $v, w \in V$, see Theorem 3.2 . 
Lemma 6.4. We have

$$
\begin{aligned}
K_{i_{1}}(z) v_{i_{2}, \ldots, i_{k+1}} & =\frac{d_{i_{2}, \ldots, i_{k+1}}}{f_{i_{1}, i_{2}, \ldots, i_{k+1}}} \sum_{\ell=1}^{k+1}(-1)^{\ell+1} a_{i_{\ell}} v_{i_{1}, \ldots, \hat{i}_{\ell}, \ldots, i_{k+1}}, \quad \text { if } i_{1} \notin\left\{i_{2}, \ldots, i_{k+1}\right\}, \\
K_{i_{1}}(z) v_{i_{1}, i_{2}, \ldots, i_{k}} & =-\sum_{m \notin\left\{i_{1}, \ldots, i_{k}\right\}} K_{i_{1}}(z) v_{m, i_{2}, \ldots, i_{k}} .
\end{aligned}
$$

Proof. The operator $K_{i}(z)$ preserve the decomposition $\operatorname{Sing} V \oplus(\operatorname{Sing} V)^{\perp}$. Hence

$$
\begin{aligned}
& K_{i_{1}}(z) v_{i_{2}, \ldots, i_{k+1}}=K_{i_{1}}(z) \pi\left(F_{i_{2}, \ldots, i_{k+1}}\right)=\pi\left(K_{i_{1}}(z) F_{i_{2}, \ldots, i_{k+1}}\right)= \\
& =\pi\left(\frac{d_{i_{2}, \ldots, i_{k+1}}}{f_{i_{1}, i_{2}, \ldots, i_{k+1}}} \sum_{\ell=1}^{k+1}(-1)^{\ell+1} a_{i_{\ell}} F_{i_{1}, \ldots, \widehat{i_{\ell}}, \ldots, i_{k+1}}\right)=\frac{d_{i_{2}, \ldots, i_{k+1}}}{f_{i_{1}, i_{2}, \ldots, i_{k+1}}} \sum_{\ell=1}^{k+1}(-1)^{\ell+1} a_{i_{\ell}} v_{i_{1}, \ldots, \widehat{i_{\ell}}, \ldots, i_{k+1}} .
\end{aligned}
$$

The differential equations (3.14) for flat sections of the Gauss-Manin connection on $\left(\mathbb{C}^{n}-\Delta\right) \times \operatorname{Sing} V \rightarrow \mathbb{C}^{n}-\Delta$ take the form

$$
\kappa \frac{\partial I}{\partial z_{j}}(z)=K_{j}(z) I(z), \quad j \in J
$$

For generic $\kappa$ all the flat sections are given by the formula

$$
I_{\gamma}(z)=\sum_{1 \leqslant i_{1}<\cdots<i_{k} \leqslant n}\left(\int_{\gamma(z)} \prod_{m \in J} f_{m}^{a_{m} / \kappa} \frac{d_{i_{1}, \ldots, i_{k}}}{f_{i_{1}} \ldots f_{i_{k}}} d t_{1} \wedge \cdots \wedge d t_{k}\right) F_{i_{1}, \ldots, i_{k}},
$$

see formula (3.12). These generic $\kappa$ can be determined more precisely from the determinant formula in [V1].

6.4. Algebra $A_{\Phi}(z)$. The master function of the arrangement $\mathcal{C}(z)$ is

$$
\Phi(z, t)=\sum_{j \in J} a_{j} \log f_{j} .
$$

The critical point equations are

$$
\frac{\partial \Phi}{\partial t_{i}}=\sum_{j \in J} b_{j}^{i} \frac{a_{j}}{f_{j}}=0, \quad i=1, \ldots, k .
$$

Introduce $H_{i}, i=1, \ldots, k$, by the formula

$$
\frac{\partial \Phi}{\partial z_{i}}=\frac{H_{i}}{\prod_{j \in J} f_{j}}
$$

The critical set is

$$
\begin{aligned}
C_{\Phi}(z) & =\left\{t \in U(\mathcal{C}(z)) \mid \frac{\partial \Phi}{\partial z_{i}}(z, t)=0, i=1, \ldots, k\right\}= \\
& =\left\{t \in U(\mathcal{C}(z)) \mid H_{i}(z, t)=0, i=1, \ldots, k\right\} .
\end{aligned}
$$

The algebra of functions on the critical set is

$$
A_{\Phi}(z)=\mathbb{C}(U(\mathcal{C}(z))) /\left\langle\frac{\partial \Phi}{\partial t_{1}}, \ldots, \frac{\partial \Phi}{\partial t_{k}}\right\rangle=\mathbb{C}(U(\mathcal{C}(z))) /\left\langle H_{1}, \ldots, H_{k}\right\rangle .
$$


Lemma 6.5. We have $\operatorname{dim} A_{\Phi}(z)=\left(\begin{array}{c}n-1 \\ k\end{array}\right)$.

Introduce elements $w_{i_{1}, \ldots, i_{k}} \in A_{\Phi}(z)$ by the formula

$$
w_{i_{1}, \ldots, i_{k}}=a_{i_{1}} \ldots a_{i_{k}}\left[\frac{d_{i_{1}, \ldots, i_{k}}}{f_{i_{1}} \ldots f_{i_{k}}}\right] \text {. }
$$

Lemma 6.6. We have $w_{i_{\sigma(1)}, \ldots, i_{\sigma(k)}}=(-1)^{\sigma} w_{i_{1}, \ldots, i_{k}}$ for $\sigma \in \Sigma_{k}$ and

$$
\sum_{i \in J} w_{i_{1}, \ldots, i_{k-1}, i}=0 \text {. }
$$

Proof. The lemma follows from the identity

$$
\begin{aligned}
& \frac{a_{i_{1}} d f_{i_{1}}}{f_{i_{1}}} \wedge \ldots \wedge \frac{a_{i_{k-1}} d f_{i_{k-1}}}{f_{i_{k-1}}} \wedge \frac{d \Phi}{\Phi}= \\
& \quad=\sum_{i \in J} a_{i_{1}} \ldots a_{k-1} a_{i} \frac{d_{i_{1}, \ldots, i_{k-1}, i}}{f_{i_{1}} \ldots f_{k-1} f_{i}} d t_{1} \wedge \cdots \wedge d t_{k}+\sum_{m \in J} d z_{j} \wedge \mu_{j},
\end{aligned}
$$

where $\mu_{j}$ are suitable $k-1$-forms.

The elements $\left[\frac{a_{i}}{f_{i}}\right], i \in J$, generate $A_{\Phi}(z)$ by Lemma 2.4 .

Lemma 6.7. For $i_{1}, \ldots, i_{k-1} \in J$, we have the following identity in $A_{\Phi}(z)$,

$$
\sum_{i \in J} d_{i, i_{1}, \ldots, i_{k-1}}\left[\frac{a_{i}}{f_{i}}\right]=0 .
$$

Denote $I=\left\{i_{1}, \ldots, i_{k-1}\right\}$. Relation (6.22) will be called the $I$-relation.

Lemma 6.8. We have in $A_{\Phi}(z)$,

$$
\begin{aligned}
& {\left[\frac{a_{i_{1}}}{f_{i_{1}}}\right] *_{z} w_{i_{2}, \ldots, i_{k+1}}=\frac{d_{i_{2}, \ldots, i_{k+1}}}{f_{i_{1}, i_{2}, \ldots, i_{k+1}}} \sum_{\ell=1}^{k+1}(-1)^{\ell+1} a_{i_{\ell}} w_{i_{1}, \ldots, \hat{i_{\ell}, \ldots, i_{k+1}}}, \quad \text { if } i_{1} \notin\left\{i_{2}, \ldots, i_{k+1}\right\},} \\
& {\left[\frac{a_{i_{1}}}{f_{i_{1}}}\right] *_{z} w_{i_{1}, i_{2}, \ldots, i_{k}}=-\sum_{m \notin\left\{i_{1}, \ldots, i_{k}\right\}}\left[\frac{a_{i_{1}}}{f_{i_{1}}}\right] *_{z} w_{m, i_{2}, \ldots, i_{k}} .}
\end{aligned}
$$

Proof. The first formula follows from the identity

$$
\frac{d f_{i_{1}}}{f_{i_{1}}} \wedge \cdots \wedge \frac{d f_{i_{k+1}}}{f_{i_{k+1}}}=\frac{d f_{i_{1}, \ldots, i_{k+1}}}{f_{i_{k+1}}} \wedge \sum_{m=1}^{k+1}(-1)^{m-1} \frac{d f_{i_{1}}}{f_{i_{1}}} \wedge \cdots \wedge \frac{\widehat{d f_{i_{m}}}}{f_{i_{m}}} \wedge \cdots \wedge \frac{d f_{i_{k+1}}}{f_{i_{k+1}}}
$$

Lemma 6.9. Fix $i_{0} \in J$. Then every monomial $M=\prod_{j \in J}\left[\frac{a_{j}}{f_{j}}\right]^{s_{j}} \in A_{\Phi}(z)$ with $\sum_{j \in J} s_{j}=k$ can be written as a linear combination of elements $w_{i_{1}, \ldots, i_{k}}$ where $i_{1}<\cdots<i_{k}$ and $i_{0} \notin$ $\left\{i_{1}, \ldots, i_{k}\right\}$ with coefficients independent of $z$. 
Proof. Let us write

$$
M=\left[\frac{a_{i_{0}}}{f_{i_{0}}}\right]^{\ell_{i_{0}}}\left[\frac{a_{j_{1}}}{f_{j_{1}}}\right]^{\ell_{j_{1}}} \ldots\left[\frac{a_{j_{m}}}{f_{j_{m}}}\right]^{\ell_{j_{m}}}
$$

where $i_{0}, j_{1}, \ldots, j_{m}$ are distinct, $\ell_{i_{0}}, \ell_{j_{1}}, \ldots, \ell_{j_{m}}$ are positive and $\ell_{i_{0}}+\ell_{j_{1}}+\cdots+\ell_{j_{m}}=k$.

If $\ell_{i_{0}}>0$, then let us decrease $\ell_{i_{0}}$ by one. For that let us use an $I$-relation of formula (6.22), where $I=\left\{p_{1}, \ldots, p_{k-1}\right\}$ is any subset which contains $j_{1}, \ldots, j_{m}$ but does not contain $i_{0}$. By using (6.22), we can write

$$
\left[\frac{a_{i_{0}}}{f_{i_{0}}}\right]^{\ell_{i_{0}}}=-\left[\frac{a_{i_{0}}}{f_{i_{0}}}\right]^{\ell_{i_{0}}-1}\left(\sum_{i \notin\left\{i_{0}, p_{1}, \ldots, p_{k-1}\right\}} \frac{d_{i, p_{1}, \ldots, p_{k-1}}}{d_{i_{0}, p_{1}, \ldots, p_{k-1}}}\left[\frac{a_{i}}{f_{i}}\right]\right) .
$$

Substituting this expression into $M$, we will present $M$ as a sum of monomials $M^{\prime}$ with the degree of $\left[\frac{a_{i_{0}}}{f_{i_{0}}}\right]$ equal to $\ell_{i_{0}}-1$. In any monomial $M^{\prime}$ the degrees of initial factors $\left[\frac{a_{j_{s}}}{f_{j_{s}}}\right]$ are the same and one new factor appears. Now to each of the monomials $M^{\prime}$ we will apply the same procedure until the monomial $\left[\frac{a_{i_{0}}}{f_{i_{0}}}\right]$ will not appear in each of the constructed monomial. Then we will decrease those degrees of $\ell_{j_{1}}, \ldots, \ell_{j_{m}}$ which are greater than one. In the end we will present $M$ as a sum of monomials of the form $\left[\frac{a_{i_{1}}}{f_{i_{1}}}\right] \ldots\left[\frac{a_{i_{k}}}{f_{i_{k}}}\right]$, where $i_{1}<\cdots<i_{k}$ and $i_{0} \notin\left\{i_{1}, \ldots, i_{k}\right\}$. Such a monomial equals $\frac{1}{d_{i_{1}, \ldots, i_{k}}} w_{i_{1}, \ldots, i_{k}}$.

Lemma 6.10. Fix $i_{0} \in J$. Then every monomial $M=\prod_{j \in J}\left[\frac{a_{j}}{f_{j}}\right]^{s_{j}} \in A_{\Phi}(z)$ can be written as a linear combination of elements $w_{i_{1}, \ldots, i_{k}}$ where $i_{1}<\cdots<i_{k}$ and $i_{0} \notin\left\{i_{1}, \ldots, i_{k}\right\}$. If $\sum_{j \in J} s_{j} \neq k$, then the coefficients of the linear combination may depend on $z$.

Proof. The lemma follows from Lemmas 6.9, 6.8, 3.4,

Theorem 6.11. Fix $i_{0} \in J$. Then the $\left(\begin{array}{c}n-1 \\ k\end{array}\right)$ elements $w_{i_{1}, \ldots, i_{k}}$ with $i_{1}<\cdots<i_{k}$ and $i_{0} \notin\left\{i_{1}, \ldots, i_{k}\right\}$, form a basis of $A_{\Phi}(z)$.

Proof. The elements $\left[\frac{a_{i}}{f_{i}}\right], i \in J$, generate $A_{\Phi}(z)$ by Lemma 2.4 . Each polynomial in $\left[\frac{a_{i}}{f_{i}}\right]$, $i \in J$, is a linear combination of $\left(\begin{array}{c}n-1 \\ k\end{array}\right)$ elements $w_{i_{1}, \ldots, i_{k}}$ with $i_{1}<\cdots<i_{k}$ and $i_{0} \notin\left\{i_{1}, \ldots, i_{k}\right\}$ by Lemma 6.10, But $\operatorname{dim} A_{\Phi}(z)=\left(\begin{array}{c}n-1 \\ k\end{array}\right)$. The theorem follows.

Theorem 6.12. For $i_{0} \in J$, the identity element $[1](z) \in A_{\Phi}(z)$ satisfies the equation

$$
\begin{aligned}
{[1](z) } & =\frac{1}{|a|^{k}} \sum_{\substack{i_{1}<\cdots<i_{k} \\
i_{0} \notin\left\{i_{1}, \ldots, i_{k}\right\}}} \frac{f_{i_{0}, i_{1}, \ldots, i_{k}}^{k}}{\prod_{m=0}^{k}(-1)^{m} d_{i_{0}, \ldots, \hat{i}_{m}, \ldots, i_{k}}} w_{i_{1}, \ldots, i_{k}}= \\
& =\frac{1}{|a|^{k}} \sum_{\substack{i_{1}<\cdots<i_{k} \\
i_{0} \notin\left\{i_{1}, \ldots, i_{k}\right\}}} \frac{\left(\sum_{m=0}^{k}(-1)^{m} z_{i_{m}} d_{i_{0}, \ldots, \widehat{i_{m}}, \ldots, i_{k}}\right)^{k}}{\prod_{m=0}^{k}(-1)^{m} d_{i_{0}, \ldots, \hat{i_{m}}, \ldots, i_{k}}} w_{i_{1}, \ldots, i_{k}} .
\end{aligned}
$$

Proof. Our goal is to prove that the decomposition of

$$
[1](z)=\frac{1}{|a|^{k}}\left(\sum_{j \in J} z_{j}\left[\frac{a_{j}}{f_{j}}\right]\right)^{k}=\frac{1}{|a|^{k}} \sum_{s_{1}+\cdots+s_{n}=k}\left(\begin{array}{c}
k \\
s_{1}, \ldots, s_{n}
\end{array}\right) \prod_{j \in J} z_{j}^{s_{j}}\left[\frac{a_{j}}{f_{j}}\right]^{s_{j}}
$$

with respect to the basis $\left(w_{i_{1}, \ldots, i_{k}}, i_{1}<\cdots<i_{k}, i_{0} \notin\left\{i_{1}, \ldots, i_{k}\right\}\right)$ equals the right hand side in ([6.27). For every monomial $\prod_{j \in J} z_{j}^{s_{j}}$ we need to show that $\left(\begin{array}{c}k \\ s_{1}, \ldots, s_{n}\end{array}\right) \prod_{j \in J}\left[\frac{a_{j}}{f_{j}}\right]^{s_{j}}$ equals 
the coefficient of that monomial in (6.27). For that we need to express $\prod_{j \in J}\left[\frac{a_{j}}{f_{j}}\right]^{s_{j}}$ as a linear combination of basis vectors. To obtain this linear combination we will eliminate from this product the factor $\left[\frac{a_{i_{0}}}{f_{i_{0}}}\right]^{s_{i}}$ and will make the powers of all other factors not greater than 1. This will be done by using the $I$-relations of formula (6.22) like in the proof of Lemma 6.9. At every step of that simplification we will use one of the $I$-relations. Although the steps of this procedure are not unique, the resulting linear combination is unique. To prove that the linear combination of basis vectors representing $\left(\begin{array}{c}k \\ s_{1}, \ldots, s_{n}\end{array}\right) \prod_{j \in J}\left[\frac{a_{j}}{f_{j}}\right]^{s_{j}}$ equals the coefficient of $\prod_{j \in J} z_{j}^{s_{j}}$ in (6.27), we will fix an arbitrary basis vector $w_{i_{1}, \ldots, i_{k}}$ and choose a particular sequence of $I$-relations so that the coefficient of $w_{i_{1}, \ldots, i_{k}}$ in the decomposition of $\left(\begin{array}{c}k \\ s_{1}, \ldots, s_{n}\end{array}\right) \prod_{j \in J}\left[\frac{a_{j}}{f_{j}}\right]^{s_{j}}$ will be equal to the coefficient of $\prod_{j \in J} z_{j}^{s_{j}} w_{i_{1}, \ldots, i_{k}}$ in (6.27).

By comparing the coefficients of a monomial $\prod_{j \in J} z_{j}^{s_{j}}$ in (6.27) and (6.28), we observe that the coefficients have common factors $\frac{1}{|a|^{k}}\left(\begin{array}{c}k \\ s_{1}, \ldots, s_{k}\end{array}\right)$, so we will ignore these common factors in our next reasonings.

Before explaining the choice of the $I$-relations for an arbitrary pair $\left(\prod_{j \in J} z_{j}^{s_{j}}, w_{i_{1}, \ldots, i_{k}}\right)$ let us consider two examples.

As the first example, we consider a monomial $M=z_{i_{1}} \ldots z_{i_{k}}, i_{1}<\cdots<i_{k}, i_{0} \notin$ $\left\{i_{1}, \ldots z_{i_{k}}\right\}$. The coefficient of $M$ in (6.28) is

$$
\begin{aligned}
{\left[\frac{a_{i_{1}}}{f_{i_{1}}}\right] } & \ldots\left[\frac{a_{i_{k}}}{f_{i_{k}}}\right]=\frac{1}{d_{i_{1}, \ldots, i_{k}}} w_{i_{1}, \ldots, i_{k}}=\frac{1}{(-1)^{0} d_{\widehat{i_{0}, i_{1}, \ldots, i_{k}}}} w_{i_{1}, \ldots, i_{k}}= \\
= & \frac{\prod_{m=1}^{k}(-1)^{m} d_{i_{0}, \ldots, \widehat{i_{m}}, \ldots, i_{k}}}{\prod_{m=0}^{k}(-1)^{m} d_{i_{0}, \ldots, \widehat{i_{m}}, \ldots, i_{k}}} w_{i_{1}, \ldots, i_{k}},
\end{aligned}
$$

which is the coefficient of $M$ in (6.27).

As the second example we consider a monomial $M=z_{i_{0}} z_{j_{i}} \ldots z_{j_{k-1}}$, where $i_{0}, j_{i}, \ldots, j_{k-1}$ are distinct. The monomial $M$ appears in (6.27) in the coefficient of a basis vector $w_{i_{1}, \ldots, i_{k}}$ if $\left\{j_{1}, \ldots, j_{k-1}\right\} \subset\left\{i_{1}, \ldots, i_{k}\right\}$. So we may assume that for some $1 \leqslant \ell \leqslant k$, we have $M=z_{i_{0}} \prod_{\substack{m=1 \\ m \neq \ell}}^{k} z_{i_{m}}$. In ([6.28), the coefficient of $M$ is $\left[\frac{a_{i_{0}}}{f_{i_{0}}}\right] \prod_{\substack{m=1 \\ m \neq \ell}}^{k}\left[\frac{a_{i_{m}}}{f_{i_{m}}}\right]$. By using the $I$ relation for $I=\left\{\widehat{i_{0}}, i_{1}, \ldots, \widehat{i_{\ell}}, \ldots, i_{k}\right\}$, we transform it into

$$
-\prod_{\substack{m=1 \\ m \neq \ell}}^{k}\left[\frac{a_{i_{m}}}{f_{i_{m}}}\right] \sum_{m \notin\left\{i_{0}, i_{1}, \ldots, \widehat{i_{\ell}}, \ldots, i_{k}\right\}} \frac{d_{m, i_{1}, \ldots, \hat{i_{\ell}}, \ldots, i_{k}}}{d_{i_{0}, i_{1}, \ldots, \hat{i_{\ell}}, \ldots, i_{k}}}\left[\frac{a_{m}}{f_{m}}\right] .
$$

We choose the summand in (6.30) corresponding to $m=i_{\ell}$. This summand is

$$
\begin{gathered}
-\prod_{\substack{m=1 \\
m \neq \ell}}^{k}\left[\frac{a_{i_{m}}}{f_{i_{m}}}\right] \frac{d_{i_{\ell}, i_{1}, \ldots, \widehat{i_{\ell}}, \ldots, i_{k}}}{d_{i_{0}, i_{1}, \ldots, \widehat{i_{\ell}}, \ldots, i_{k}}}\left[\frac{a_{i_{\ell}}}{f_{i_{\ell}}}\right]=\prod_{m=1}^{k}\left[\frac{a_{i_{m}}}{f_{i_{m}}}\right] \frac{d_{i_{1}, \ldots, i_{\ell}, \ldots, i_{k}}}{(-1)^{\ell} d_{i_{0}, i_{1}, \ldots, \widehat{i_{\ell}}, \ldots, i_{k}}}= \\
=\frac{\prod_{\substack{m=0 \\
m \neq \ell}}^{k}(-1)^{m} d_{i_{0}, \ldots, \widehat{i_{m}}, \ldots, i_{k}}}{\prod_{m=0}^{k}(-1)^{m} d_{i_{0}, \ldots, \widehat{i_{m}}, \ldots, i_{k}}} w_{i_{1}, \ldots, i_{\ell}, \ldots, i_{k}},
\end{gathered}
$$

which is the coefficient of $M w_{i_{1}, \ldots, i_{\ell}, \ldots, i_{k}}$ in (6.27). 
Now let $M$ be an arbitrary monomial of degree $k$ in variables $z_{1}, \ldots, z_{n}$. The monomial $M$ appears in (6.27) in the coefficient of a vector $w_{i_{1}, \ldots, i_{k}}$ if there is a subset $\left\{p_{1}, \ldots, p_{r}\right\} \subset$ $\{1, \ldots, k\}$ such that $M=z_{i_{0}}^{s_{0}} \prod_{m=1}^{r} z_{i_{p_{m}}}^{s_{m}}, \sum_{m=0}^{r} s_{m}=k$, and all numbers $s_{1}, \ldots, s_{r}$ are positive. Denote $\left\{q_{1}, \ldots, q_{k-r}\right\}=\{1, \ldots, k\}-\left\{p_{1}, \ldots, p_{r}\right\}$, the complement.

In (6.28), the coefficient of $M$ is $P=\left[\frac{a_{i_{0}}}{f_{i_{0}}}\right]^{s_{0}} \prod_{m=1}^{r}\left[\frac{a_{i_{m}}}{f_{i_{m}}}\right]^{s_{m}}$. To express this product as a linear combination of the basis vectors we need to apply to this product $I$-relations $k-r$ times. To calculate the coefficient of $w_{i_{1}, \ldots, i_{k}}$ we first apply the $I$-relation with $I=$ $\left\{\widehat{i_{0}}, i_{1}, \ldots, \widehat{i_{q_{1}}}, \ldots, i_{k}\right\} \subset\left\{i_{0}, \ldots, i_{k}\right\}$ and decrease the degree of $\left[\frac{a_{i_{0}}}{f_{i_{0}}}\right]$ by 1 ,

$$
P=-\left[\frac{a_{i_{0}}}{f_{i_{0}}}\right]^{s_{0}-1} \prod_{m=1}^{r}\left[\frac{a_{i_{p_{m}}}}{f_{i_{p_{m}}}}\right]^{s_{m}} \sum_{m \notin\left\{i_{0}, \ldots, \widehat{\hat{q}_{1}}, \ldots, i_{k}\right\}} \frac{d_{m, \widehat{i_{0}}, i_{1}, \ldots, \widehat{i_{q_{1}}}, \ldots, i_{k}}}{d_{i_{0}, \widehat{i_{0}}, i_{1}, \ldots, \hat{q_{q_{1}}}, \ldots, i_{k}}}\left[\frac{a_{m}}{f_{m}}\right] .
$$

In the next steps we will simplify further the first factors of this expression. After the future simplifications the only term of this sum that can give $w_{i_{1}, \ldots, i_{k}}$ is the term with $m=i_{q_{1}}$, which is

$$
\begin{aligned}
& -\left[\frac{a_{i_{0}}}{f_{i_{0}}}\right]^{s_{0}-1} \prod_{m=1}^{r}\left[\frac{a_{i_{p_{m}}}}{f_{i_{p_{m}}}}\right]^{s_{m}} \frac{d_{i_{q_{1}}, \widehat{i_{0}}, i_{1}, \ldots, \widehat{i_{q_{1}}}, \ldots, i_{k}}}{d_{i_{0}, \widehat{i_{0}}, i_{1}, \ldots, \widehat{i_{q_{1}}}, \ldots, i_{k}}}\left[\frac{a_{i_{q_{1}}}}{f_{i_{q_{1}}}}\right]= \\
& =\left[\frac{a_{i_{0}}}{f_{i_{0}}}\right]^{s_{0}-1} \prod_{m=1}^{r}\left[\frac{a_{i_{p_{m}}}}{f_{i_{p_{m}}}}\right]^{s m} \frac{d_{\widehat{i_{0}}, i_{1}, \ldots, i_{k}}}{(-1)^{q_{1}} d_{i_{0}, i_{1}, \ldots, \widehat{i_{1}}}, \ldots, i_{k}}\left[\frac{a_{i_{q_{1}}}}{f_{i_{q_{1}}}}\right] .
\end{aligned}
$$

We will call this term the main term. Now we apply the $I$-relation with $I=\left\{\widehat{i_{0}}, i_{1}, \ldots\right.$, $\left.\widehat{i_{q_{2}}}, \ldots, i_{k}\right\} \subset\left\{i_{0}, \ldots, i_{k}\right\}$ and again decrease the degree of $\left[\frac{a_{i_{0}}}{f_{i_{0}}}\right]$ by one. After the second step the only term of the obtained sum that may produce the vector $w_{i_{1}, \ldots, i_{k}}$ is the term

$$
\left[\frac{a_{i_{0}}}{f_{i_{0}}}\right]^{s_{0}-1} \prod_{m=1}^{r}\left[\frac{a_{i_{p_{m}}}}{f_{i_{p_{m}}}}\right]^{s_{m}} \frac{d_{\widehat{i_{0}, i_{1}, \ldots, i_{k}}}}{(-1)^{i_{q_{1}}} d_{i_{0}, i_{1}, \ldots, \hat{i}_{q_{1}}, \ldots, i_{k}}}\left[\frac{a_{i_{q_{1}}}}{f_{i_{q_{1}}}}\right] \frac{d_{\widehat{i_{0}, i_{1}, \ldots, i_{k}}}}{(-1)^{q_{2}} d_{i_{0}, i_{1}, \ldots, \widehat{i_{q_{2}}}, \ldots, i_{k}}}\left[\frac{a_{i_{q_{2}}}}{f_{i_{q_{2}}}}\right] .
$$

This will be our main term after two steps of the simplifying procedure. We will repeat this procedure to kill all factors $\left[\frac{a_{i_{0}}}{f_{i_{0}}}\right]$. After $s_{i_{0}}$ steps the main term will be

$$
\prod_{m=1}^{r}\left[\frac{a_{i_{p_{m}}}}{f_{i_{p_{m}}}}\right]^{s_{m}} \prod_{m=1}^{s_{0}} \frac{d_{\widehat{i_{0}, i_{1}, \ldots, i_{k}}}}{(-1)^{q_{m}} d_{i_{0}, i_{1}, \ldots, i_{q_{m}}, \ldots, i_{k}}}\left[\frac{a_{i_{q_{m}}}}{f_{i_{q_{2}}}}\right] .
$$

Now we will apply the $I$-relation with $I=\left\{i_{0}, \ldots, \widehat{i_{p_{1}}}, \ldots, \widehat{i_{q_{0}+1}}, \ldots, i_{k}\right\} \subset\left\{i_{0}, \ldots, i_{k}\right\}$ and decrease the degree of $\left[\frac{a_{i_{1}}}{f_{i_{1}}}\right]$ by one. After this procedure the main term will be

$$
\left[\frac{a_{i_{p_{1}}}}{f_{i_{p_{1}}}}\right]^{s_{1}-1} \prod_{m=2}^{r}\left[\frac{a_{i_{p_{m}}}}{f_{i_{p_{m}}}}\right]^{s_{m}} \prod_{m=1}^{s_{0}} \frac{d_{\widehat{i_{0}}, i_{1}, \ldots, i_{k}}}{(-1)^{q_{m}} d_{i_{0}, i_{1}, \ldots, \hat{q_{m}}, \ldots, i_{k}}}\left[\frac{a_{i_{q_{m}}}}{f_{i_{q_{2}}}}\right] \times \frac{(-1)^{p_{1}} d_{i_{0}, i_{1}, \ldots, \widehat{i_{p_{1}}}, \ldots, i_{k}}}{(-1)^{q_{s_{0}+1}} d_{i_{0}, i_{1}, \ldots, \hat{q_{s_{0}+1}}, \ldots, i_{k}}}\left[\frac{a_{i_{q_{s_{0}+1}}}}{f_{i_{q_{s_{0}+1}}}}\right] .
$$

Now we will be decreasing the degree of $\left[\frac{a_{j_{1}}}{f_{j_{1}}}\right]$ to make it 1 . Then we will continue this procedure of simplification, which will end with the main term

$$
\left(d_{\widehat{i_{0}}, i_{1}, \ldots, i_{k}} \prod_{m=1}^{k}\left[\frac{a_{i_{m}}}{f_{i_{m}}}\right]\right) \frac{\left((-1)^{0} d_{\widehat{i_{0}}, i_{1}, \ldots, i_{k}}\right)^{s_{0}} \prod_{m=1}^{r}\left((-1)^{p_{m}} d_{i_{0}, i_{1}, \ldots, \widehat{i_{p_{m}}}, \ldots, i_{k}}\right)^{s_{m}}}{\prod_{m=0}^{k}(-1)^{m} d_{i_{0}, \ldots, \widehat{i_{m}}, \ldots, i_{k}}} .
$$


After replacing the first factor $d_{\widehat{i_{0}}, i_{1}, \ldots, i_{k}} \prod_{m=1}^{k}\left[\frac{a_{i_{m}}}{f_{i_{m}}}\right]$ with $w_{i_{1}, \ldots, i_{k}}$ we observe that the second factor equals the coefficient of $M w_{i_{1}, \ldots, i_{k}}$ in (6.27). The theorem is proved.

6.5. Canonical isomoprhism. The set of vectors $v_{i_{1}, \ldots, i_{k}}, 1<i_{1}<\cdots<i_{k} \leqslant n$, is a basis of Sing $V$, by Lemma 6.2. For $z \in \mathbb{C}^{n}-\Delta$, the set of vectors $w_{i_{1}, \ldots, i_{k}}, 1<i_{1}<\cdots<i_{k} \leqslant n$, is a basis of $A_{\Phi}(z)$, by Theorem 6.11.

Theorem 6.13. For $z \in \mathbb{C}^{n}-\Delta$, the matrix of the canonical isomorphism $\alpha(z): A_{\Phi}(z) \rightarrow$ Sing $V$ with respect to these bases does not depend on $z$.

Proof. For $1<m_{1}<\cdots<m_{k} \leqslant n$ and $1 \leqslant i_{1}<\cdots<i_{k} \leqslant n$ denote

$$
B_{i_{1}, \ldots, i_{k}}=\sum_{p \in C_{\Phi}(z)} \operatorname{Res}_{p} \frac{1}{\prod_{j=1}^{k} f_{m_{j}}} \frac{1}{\prod_{j=1}^{k} f_{i_{j}}} \frac{\prod_{\ell \in J} f_{\ell}^{k}}{\prod_{i=1}^{k} H_{i}} .
$$

Then

$$
\alpha(z)\left(w_{m_{1}, \ldots, m_{k}}\right)=\sum_{1 \leqslant i_{1}<\cdots<i_{k} \leqslant n} \frac{d_{m_{1}, \ldots, m_{k}} d_{i_{1}, \ldots, i_{k}} \prod_{j=1}^{k} a_{m_{j}}}{(2 \pi \sqrt{-1})^{k}} B_{i_{1}, \ldots, i_{k}} F_{i_{1}, \ldots, i_{k}},
$$

see formulas (2.19), (2.22) . In order to prove the theorem we need to show that every $B_{i_{1}, \ldots, i_{k}}$ does not depend on $z$.

The differential form

$$
\omega=\frac{1}{\prod_{j=1}^{k} f_{m_{j}}} \frac{1}{\prod_{j=1}^{k} f_{i_{j}}} \frac{\prod_{\ell \in J} f_{\ell}^{k}}{\prod_{i=1}^{k} H_{i}} d t_{1} \wedge \cdots \wedge d t_{k}
$$

has poles only on the hypersurfaces $H_{i}=0, i=1, \ldots, k$. The poles are of first order. To calculate $B_{i_{1}, \ldots, i_{k}}$, we need to take the residue $\psi=\operatorname{Res}_{H_{i}=0, i=1, \ldots, k-1} \omega$ of the form $\omega$ at the curve $\mathcal{C}=\left\{H_{i}=0, i=1, \ldots, k-1\right\}$ and then take the residue of the form $\psi$ on the curve $\mathcal{C}$ at the points where $H_{k}=0$. This is the same as if we took with minus sign the residue at infinity of the form $\psi$ on the curve $\mathcal{C}$. That residue at infinity (up to sign) can be obtained differently in two steps. First we may take the residue of $\omega$ at the hyperplane at infinity (denote the residue by $\varphi$ ) and then take the residue of $\varphi$ at the points of the set $\left\{H_{i}=0, i=1, \ldots, k-1\right\}$.

So to calculate $B_{i_{1}, \ldots, i_{k}}$ we first calculate $\varphi$. The coordinates at infinity are $u_{1}=t_{1} / t_{k}, \ldots$, $u_{k-1}=t_{k-1} / t_{k}, u_{k}=1 / t_{k}$. We have $f_{m}=\left(b_{m}^{1} u_{1}+\cdots+b_{m}^{k-1} u_{k-1}+b_{m}^{k}+z_{m} u_{k}\right) / u_{k}$. Denote $\tilde{f}_{m}\left(u_{1}, \ldots, u_{k-1}\right)=b_{m}^{1} u_{1}+\cdots+b_{m}^{k-1} u_{k-1}+b_{m}^{k}$. For $i=1, \ldots, k$, we have $H_{i}\left(u_{1} / u_{k}, \ldots\right.$, $\left.u_{k-1} / u_{k}, 1 / u_{k}\right)=\hat{H}_{i}\left(u_{1}, \ldots, u_{k-1}, u_{k}\right) / u_{k}^{n-1}$, where $\hat{H}_{i}\left(u_{1}, \ldots, u_{k-1}, u_{k}\right)$ is a polynomial. Denote $\tilde{H}_{i}\left(u_{1}, \ldots, u_{k-1}\right)=\hat{H}_{i}\left(u_{1}, \ldots, u_{k-1}, 0\right)$. The polynomial $\tilde{H}_{i}\left(u_{1}, \ldots, u_{k-1}\right)$ does not depend on $z$.

We have $d t_{1} \wedge \cdots \wedge d t_{k}=-\frac{1}{u_{k}^{k+1}} d u_{1} \wedge \cdots \wedge d u_{k}$. By counting all orders of $u_{k}$ in factors of $\omega$ we conclude that the form $\omega$ has the first order pole at the hyperplane at infinity. The residue $\varphi$ of $\omega$ at the infinite hyperplane equals

$$
\pm 2 \pi \sqrt{-1} \frac{1}{\prod_{j=1}^{k} \tilde{f}_{m_{j}}} \frac{1}{\prod_{j=1}^{k} \tilde{f}_{i_{j}}} \frac{\prod_{\ell \in J} \tilde{f}_{\ell}^{k}}{\prod_{i=1}^{k} \tilde{H}_{i}} d u_{1} \wedge \cdots \wedge d u_{k-1} .
$$


This form does not depend on $z$. Now we are supposed to take the sum of residues of $\varphi$ at the points of the set $\left\{\tilde{H}_{i}=0, i=1, \ldots, k-1\right\}$ and the polynomials $\tilde{H}_{i}$ also do not depend on $z$. Hence $B_{i_{1}, \ldots, i_{k}}$ does not depend on $z$. The theorem is proved.

Define the naive isomorphism $\nu(z): A_{\Phi}(z) \rightarrow \operatorname{Sing} V$ by the formula

$$
\nu(z): w_{i_{1}, \ldots, i_{k}} \mapsto v_{i_{1}, \ldots, i_{k}}
$$

for all $i_{1}, \ldots, i_{k} \in J$.

Lemma 6.14. The map $\nu(z)$ is an isomorphism of vector spaces and for every $i \in J$ and $w \in A_{\Phi}(z)$ we have

$$
\nu(z)\left[\frac{a_{i}}{f_{i}}\right] *_{z} w=K_{i}(z) \nu(z)(w) .
$$

Proof. The map $\nu(z)$ is an isomorphism by Lemma 6.2 and Theorem 6.11, Formula (6.37) holds by Lemmas 6.4 and 6.8 .

Introduce the linear isomorphism

$$
\zeta=\alpha(z) \nu(z)^{-1}: \operatorname{Sing} V \rightarrow \operatorname{Sing} V .
$$

By Theorem 6.13 and Lemma 6.14 the isomorphism $\zeta$ does not depend on $z$. By Theorems 6.13 and 3.5 the isomorphism $\zeta$ commutes with the action of operators $K_{i}(z)$ for all $i \in J$ and $z \in \mathbb{C}^{n}-\Delta,\left[K_{i}(z), \zeta\right]=0$.

Theorem 6.15. The isomorphism $\zeta$ is a scalar operator.

Proof. By Lemma 4.3 in [V5], the eigenvalues of the operators $K_{i}(z)$ separate the eigenvectors. The theorem follows from the fact that the operators $K_{i}(z)$ have too many eigenvectors, and $\zeta$ must preserve all of them. More precisely, let $i_{1}, \ldots, i_{k+1} \in J$ be distinct. Assume that $z \in \mathbb{C}^{n}-\Delta$ tends to a generic point $z^{0}$ of the hyperplane defined by the equation $f_{i_{1}, \ldots, i_{k+1}}=0$. It follows from Lemma 6.4 that the vector

$$
x_{i_{1}, \ldots, i_{k+1}}=\sum_{\ell=1}^{k+1}(-1)^{\ell+1} a_{i_{\ell}} v_{i_{1}, \ldots, \widehat{\hat{\ell}_{\ell}}, \ldots, i_{k+1}} \in \operatorname{Sing} V
$$

is the limit of an eigenvector of operators $K_{i}(z)$ as $z \rightarrow z^{0}$. Hence, $x_{i_{1}, \ldots, i_{k+1}}$ is an eigenvector of $\zeta$. It is easy to see that the vectors $x_{i_{1}, \ldots, i_{k+1}}$ generate $\operatorname{Sing} V$ and for distinct $i_{1}, \ldots, i_{k+2}$ we have

$$
\sum_{\ell=1}^{k+2}(-1)^{\ell} a_{\ell} x_{i_{1}, \ldots, \hat{i_{\ell}}, \ldots, i_{k=2}}=0 .
$$

This equation implies that $\zeta$ is a scalar operator on the subspace generated by the vectors $x_{i_{1}, \ldots, \widehat{i_{\ell}}, \ldots, i_{k=2}}, \ell=1, \ldots, k+2$, and this fact implies that $\zeta$ is a scalar operator on $\operatorname{Sing} V$.

Corollary 6.16. There exists $c \in \mathbb{C}^{\times}$such that $\alpha(z)=c \nu(z)$, that is,

$$
\alpha(z): w_{i_{1}, \ldots, i_{k}} \mapsto c v_{i_{1}, \ldots, i_{k}}
$$

for all $i_{1}, \ldots, i_{k} \in J$. 
One may expect that $c=(-1)^{k}$, see Theorems 4.8 and 5.15 .

The canonical isomorphism $\alpha(z)$ induces an algebra structure on $\operatorname{Sing} V$ depending on $z \in \mathbb{C}^{n}-\Delta$.

Corollary 6.17. For any $i_{0} \in J$, the identity element $\{1\}(z)$ of that algebra structure satisfies the equation

$$
\{1\}(z)=\frac{c}{|a|^{k}} \sum_{\substack{i_{1}<\cdots<i_{k} \\ i_{0} \notin\left\{i_{1}, \ldots, i_{k}\right\}}} \frac{f_{i_{0}, i_{1}, \ldots, i_{k}}^{k}}{\prod_{m=0}^{k}(-1)^{m} d_{i_{0}, \ldots, \widehat{i_{m}}, \ldots, i_{k}}} v_{i_{1}, \ldots, i_{k}},
$$

where $c$ is defined in Corollary 6.16.

Theorem 6.18. Conjectures 3.7 and 3.8 hold for this family of arrangements.

Proof. Conjecture 3.8 is a direct corollary of Theorem 6.13.

Lemma 6.19. For $r \leqslant k$ and $m_{1}, \ldots, m_{r} \in J$, we have

$$
\frac{\partial^{r}\{1\}}{\partial z_{m_{1}} \ldots \partial z_{m_{r}}}(z)=\frac{k(k-1) \ldots(k-r+1)}{|a|^{r}} \alpha(z)\left(\prod_{i=1}^{r}\left[\frac{a_{m_{i}}}{f_{m_{i}}}\right]\right) .
$$

Proof. The proof is by induction on $r$. For $r=0$, the statement is true: $\{1\}=\{1\}$. Assuming the statement is true for some $r$, we prove the statement for $r+1$. We have

$$
\begin{aligned}
& \frac{\partial}{\partial z_{j}} \frac{\partial^{r}\{1\}}{\partial z_{m_{1}} \ldots \partial z_{m_{r}}}(z)=\frac{\partial}{\partial z_{j}} \frac{k(k-1) \ldots(k-r+1)}{|a|^{r}} \alpha(z)\left(\prod_{i=1}^{r}\left[\frac{a_{m_{i}}}{f_{m_{i}}}\right]\right)= \\
& =\frac{\partial}{\partial z_{j}} \frac{k(k-1) \ldots(k-r+1)}{|a|^{r}} \alpha(z)\left(\prod_{i=1}^{r}\left[\frac{a_{m_{i}}}{f_{m_{i}}}\right] \frac{1}{|a|^{k-r}}\left(\sum_{i \in J} z_{i}\left[\frac{a_{i}}{f_{i}}\right]\right)^{k-r}\right)= \\
& =\frac{k-r}{|a|} \alpha(z)\left(\left[\frac{a_{j}}{f_{j}}\right]\right) *_{z} \frac{k(k-1) \ldots(k-r+1)}{|a|^{r}} \alpha(z)\left(\prod_{i=1}^{r}\left[\frac{a_{m_{i}}}{f_{m_{i}}}\right] \times\right. \\
& \left.\times \frac{1}{|a|^{k-r-1}}\left(\sum_{i \in J} z_{i}\left[\frac{a_{i}}{f_{i}}\right]\right)^{k-r-1}\right)=\frac{k(k-1) \ldots(k-r+1)(k-r)}{|a|^{r+1}} \alpha(z)\left(\left[\frac{a_{j}}{f_{j}}\right] \prod_{i=1}^{r}\left[\frac{a_{m_{i}}}{f_{m_{i}}}\right]\right) .
\end{aligned}
$$

Let us finish the proof of Conjecture 3.7. We have

$$
\begin{aligned}
\frac{\partial}{\partial z_{j}} \frac{\partial^{r}\{1\}}{\partial z_{m_{1}} \ldots \partial z_{m_{r}}}(z)= & \frac{k-r}{|a|} \alpha(z)\left(\left[\frac{a_{j}}{f_{j}}\right]\right) *_{z} \frac{k(k-1) \ldots(k-r+1)}{|a|^{r}} \alpha(z)\left(\prod_{i=1}^{r}\left[\frac{a_{m_{i}}}{f_{m_{i}}}\right]\right)= \\
= & \frac{k-r}{|a|} K_{j}(z) \frac{\partial^{r}\{1\}}{\partial z_{m_{1}} \ldots \partial z_{m_{r}}}(z) .
\end{aligned}
$$

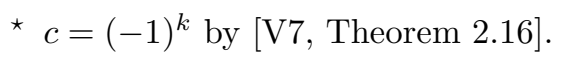


Introduce the potential function of second kind

$$
\tilde{P}\left(z_{1}, \ldots, z_{n}\right)=\frac{c^{2}}{(2 k) !} \sum_{1 \leqslant i_{1}<\cdots<i_{k+1} \leqslant n} \frac{\prod_{\ell=1}^{k+1} a_{i_{\ell}}}{\prod_{\ell=1}^{k+1} d_{i_{1}, \ldots, \widehat{i_{\ell}, \ldots, i_{k+1}}}^{2}} f_{i_{1}, \ldots, i_{k+1}}^{2 k} \log f_{i_{1}, \ldots, i_{k+1}}
$$

where $c$ is the constant defined in Corollary 6.16,

Theorem 6.20. For any $m_{0}, \ldots, m_{2 k} \in J$, we have

$$
\frac{\partial^{2 k+1} \tilde{P}}{\partial z_{m_{0}} \cdots \partial z_{m_{2 k}}}(z)=(-1)^{k}\left(\left[\frac{a_{m_{0}}}{f_{m_{0}}}\right] *_{z} \cdots *_{z}\left[\frac{a_{m_{2 k}}}{f_{m_{2 k}}}\right],[1](z)\right)_{z} .
$$

Theorem 6.20 proves Conjecture 3.14 for this family of arrangements.

If $m_{1}, \ldots, m_{k}$ are distinct and $m_{k+1}, \ldots, m_{2 k}$ are distinct, equation (6.45) takes the form

$$
c^{2} S^{(a)}\left(K_{m_{0}}(z) v_{m_{1}, \ldots, m_{k}}, v_{m_{k+1}, \ldots, m_{2 k}}\right)=d_{m_{1}, \ldots, m_{k}} d_{m_{k+1}, \ldots, m_{2 k}} \frac{\partial^{2 k+1} \tilde{P}}{\partial z_{m_{0}} \ldots \partial z_{m_{2 k}}}(z) .
$$

Corollary 6.21. The matrix elements of the operators $K_{i}(z)$ with respect to the (combinatorially constant) vectors $v_{i_{1}, \ldots, i_{k}}$ are described by the $(2 k+1)$-st derivatives of the potential function of second kind.

Notice that

$$
c^{2} S^{(a)}\left(v_{m_{1}, \ldots, m_{k}}, v_{m_{k+1}, \ldots, m_{2 k}}\right)=d_{m_{1}, \ldots, m_{k}} d_{m_{k+1}, \ldots, m_{2 k}} \frac{|a|^{2 k}}{(2 k) !} \frac{\partial^{2 k} P}{\partial z_{m_{1}} \ldots \partial z_{m_{2 k}}}(z),
$$

where $P(z)$ is the potential function of first kind, see Theorem 3.11 .

Proof. We have the $I$-relation $\sum_{j \in J} d_{j, i_{1}, \ldots, i_{k-1}}\left[\frac{a_{j}}{f_{j}}\right]=0$ for any $i_{1}, \ldots, i_{k-1} \in J$, see (6.22), and the relation

$$
\sum_{j \in J} d_{i, i_{1}, \ldots, i_{k-1}} \frac{\partial}{\partial z_{j}} \frac{\partial^{2 k} \tilde{P}}{\partial z_{m_{1}} \ldots \partial z_{m_{2 k}}}(z)=0
$$

for any $m_{1}, \ldots, m_{2 k}, i_{1}, \ldots, i_{k-1} \in J$. By using these two relations and by reordering the set $J$ if necessary, we can reduce formula (6.45) to the case in which $\left(m_{1}, \ldots, m_{k}\right)$ are distinct, $\left(m_{k+1}, \ldots, m_{2 k}\right)$ are distinct, and $m_{0} \notin\left\{m_{1}, \ldots, m_{k}\right\}$. After that we need to check identity (6.46). That is done by direct calculation of the left and right hand sides, cf. the proof of Theorem 5.27 .

For example, the most difficult case is if $\left(m_{0}, \ldots, m_{2 k}\right)=(k+1,1, \ldots, k, 1, \ldots, k)$. Then

$$
\frac{\partial^{2 k+1} \tilde{P}}{\partial z_{m_{0}} \ldots \partial z_{m_{2 k}}}(z)=c^{2} \frac{\prod_{m=1}^{k+1} a_{m}}{(-1)^{k} d_{1, \ldots, k} f_{1, \ldots, k+1}}
$$


and

$$
\begin{aligned}
& (-1)^{k}\left(\left[\frac{a_{m_{0}}}{f_{m_{0}}}\right] *_{z} \cdots *_{z}\left[\frac{a_{m_{2 k}}}{f_{m_{2 k}}}\right],[1](z)\right)_{z}=c^{2} \frac{1}{d_{1, \ldots, k}^{2}} S^{(a)}\left(K_{k+1}(z) v_{1, \ldots, k}, v_{1, \ldots, k}\right)= \\
& =c^{2} \frac{1}{d_{1, \ldots, k} f_{k+1,1, \ldots, k}} S^{(a)}\left(a_{k+1} v_{1, \ldots, k}+\sum_{\ell=1}^{k}(-1)^{\ell} a_{\ell} v_{k+1,1, \ldots, \hat{\ell}, \ldots, k}, v_{1, \ldots, k}\right)= \\
& =c^{2} \frac{1}{d_{1, \ldots, k} f_{k+1,1, \ldots, k}}\left(\frac{\prod_{m=1}^{k+1} a_{m}}{|a|} \sum_{\ell \notin\{1, \ldots, k\}} a_{\ell}+\frac{\prod_{m=1}^{k+1} a_{m}}{|a|} \sum_{\ell \in\{1, \ldots, k\}} a_{\ell}\right)= \\
& =c^{2} \frac{1}{d_{1, \ldots, k} f_{k+1,1, \ldots, k}} \prod_{m=1}^{k+1} a_{m} .
\end{aligned}
$$

\section{REFERENCES}

[AGV] V.I. Arnol'd, S.M. Gusein-Zade, A.N. Varchenko, Singularities of Differential Maps, Vol. I, Nauka, Moscow, 1982; also Birkhauser, 1985

[BMO] A. Braverman, D. Maulik, A. Okounkov, Quantum cohomology of the Springer resolution, Adv. Math. 227 (2011), no. 1, 421-458

[Do] A. Douai, A canonical Frobenius structure, Math. Z. 261 (2009), no. 3, 625-648

[D1] B. Dubrovin, Geometry of 2D topological field theories, Integrable Systems and Quantum Groups, ed. Francaviglia, M. and Greco, S.. Springer lecture notes in mathematics, 1620, 120-348

[D2] B. Dubrovin, On almost duality for Frobenius manifolds, Geometry, topology, and mathematical physics, 75-132, Amer. Math. Soc. Transl. Ser. 2, 212, Amer. Math. Soc., Providence, RI, 2004

[EFK] P. Etingof, I. Frenkel, A. Kirillov, Lectures on representation theory and KnizhnikZamolodchikov equations, Mathematical Surveys and Monographs, 58, AMS, 1998

[FaV] M. Falk, A. Varchenko, The contravariant form on singular vectors of a projective arrangement, In Configuration Spaces, Geometry, Combinatorics and Topology" edited by A. Bjorner, F. Cohen, C. De Concini, C. Procesi, and M. Salvetti, CRM Series 2012, 255-272

[FSV1] B. Feigin, V. Schechtman, A. Varchenko, On algebraic equations satisfied by hypergeometric correlators in WZW models, I, Comm. Math. Phys. 163 (1994), No. 1, 173-184

[FSV2] B. Feigin, V. Schechtman, A. Varchenko, On algebraic equations satisfied by hypergeometric correlators in WZW models, II, Comm. Math. Phys. 170, No. 1, 219-247

[FV] M. Feigin, A. Veselov, Logarithmic Frobenius structures and Coxeter discriminants, Adv. Math. 212 (2007), no. 1, 143-162

[GRTV] V. Gorbounov, R. Rimanyi, V. Tarasov, A. Varchenko, Cohomology of the cotangent bundle of a flag variety as a Yangian Bethe algebra, Journal of Geometry and Physics (2013), pp. 56-86

[GH] Ph. Griffiths, J. Harris, Principles of Algebraic Geometry, Wiley, 1994

$[\mathrm{HM}]$ C. Hertling and Yu.I. Manin, Unfoldings of meromorphic connections and a construction of Frobenius manifolds, Frobenius manifolds, Aspects of Math. 36 (2004) 113-144

[M] Y.I. Manin, Frobenius manifolds, quantum cohomology, and moduli spaces, American Mathematical Society Colloquium Publications, vol. 47, AMS, Providence, RI, 1999

[MTV] E. Mukhin, V. Tarasov, A. Varchenko, Three sides of the geometric Langlands correspondence for $g_{N}$ Gaudin model and Bethe vector averaging maps, Arrangements of hyperplanes - Sapporo 2009, 475-511, Adv. Stud. Pure Math., 62, Math. Soc. Japan, Tokyo, 2012

[OT1] P. Orlik, H. Terao, The number of critical points of a product of powers of linear functions, Invent. Math. 120 (1995), no. 1, 1-14 
[OT2] P. Orlik, H. Terao, Arrangements and Hypergeometric Integrals, MSJ Memoirs, vol. 9, Mathematical Society of Japan, Tokyo, 2001

[RV] N. Reshetikhin and A. Varchenko, Quasiclassical asymptotics of solutions to the KZ equations, Geometry, Topology and Physics for R. Bott, Intern. Press, 1995, 293-322

[Ri] A. Riley, Frobenius manifolds: caustic submanifolds and discriminant almost duality, University of Hull $\mathrm{PhD}$ thesis, 2007

[Si] R. Silvotti, On a conjecture of Varchenko, Invent. Math. 126 (1996), no. 2, 235-248

[St] I.A.B. Strachan, Frobenius manifolds: natural submanifolds and induced bi-Hamiltonian structures, Differential Geom. Appl. 20 (2004), no. 1, 67-99

[SV] V.Schechtman and A. Varchenko, Arrangements of Hyperplanes and Lie Algebra Homology, Invent. Math. Vol. 106 (1991), 139-194

[V1] A. Varchenko, Beta-Function of Euler, Vandermonde Determinant, Legendre Equation and Critical Values of Linear Functions of Configuration of Hyperplanes, I. Izv. Akademii Nauk USSR, Seriya Mat., 53:6 (1989), 1206-1235

[V2] A. Varchenko, Multidimensional Hypergeometric Functions and Representation Theory of Lie Algebras and Quantum Groups, Advanced Series in Mathematical Physics, Vol. 21, World Scientific, 1995

[V3] A. Varchenko, Critical points of the product of powers of linear functions and families of bases of singular vectors, Compos. Math. 97 (1995), 385-401.

[V4] A. Varchenko, Special functions, KZ type equations, and Representation theory, CBMS, Regional Conference Series in Math., n. 98 (2003), AMS

[V5] A. Varchenko, Bethe Ansatz for Arrangements of Hyperplanes and the Gaudin Model, Mosc. Math. J. 6 (2006), no. 1, 195-210, 223-224

[V6] A. Varchenko, Quantum integrable model of an arrangement of hyperplanes, SIGMA Symmetry Integrability Geom. Methods Appl. 7 (2011), Paper 032, 55 pp.

[V7] A. Varchenko, Characteristic variety of the Gauss-Manin differential equations and critical set of the master function, in preparation

[Y] S. Yuzvinsky, Cohomology of the Brieskorn-Orlik-Solomon algebras, Communications in Algebra 23 (1995), 5339-5354 